Frauenklinik und Poliklinik

der Technischen Universität München

Klinikum rechts der Isar

(Direktorin: Univ.-Prof. Dr. M. B. Kiechle)

\title{
Einfluss von Betamethason auf die fetale Herzfrequenzvariabilität und Vergleich zweier Registrierungsmethoden: CTG und fetales EKG
}

\section{Anna- Luise Setter}

Vollständiger Abdruck der von der Fakultät für Medizin der Technischen Universität München zur Erlangung des akademischen Grades eines Doktors der Medizin genehmigten Dissertation.

Vorsitzender: Univ.-Prof. Dr. E. J. Rummeny Prüfer der Dissertation:

1. Univ.-Prof. Dr. K.-Th. M. Schneider

2. apl. Prof. Dr. Dr. H. Kaemmerer

Die Dissertation wurde am 30.12.2014 bei der Technischen Universität München eingereicht und durch die Fakultät für Medizin am 17.06.2015 angenommen. 


\section{Inhaltsverzeichnis}

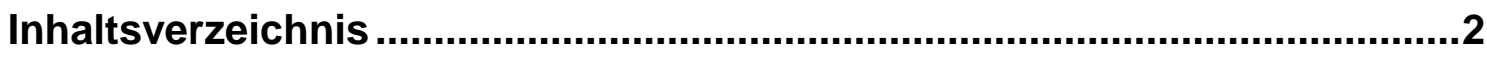

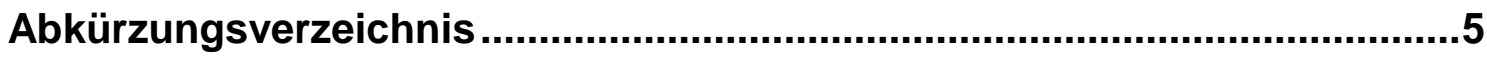

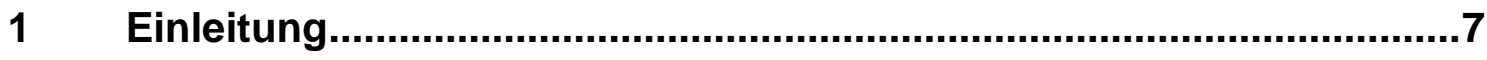

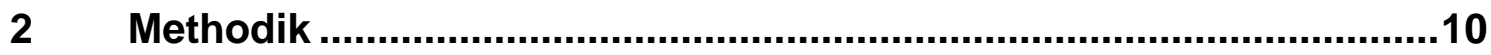

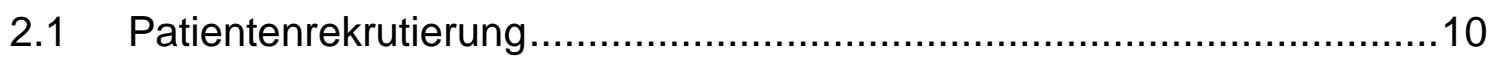

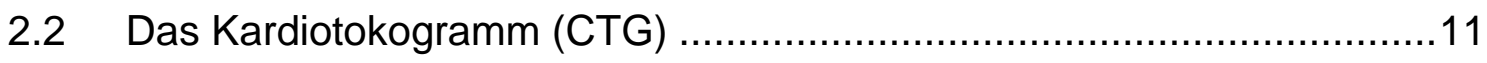

2.2.1 Berechnung der Parameter der Dawes-Redman Analyse .....................14

2.2.2 Berechnung der Kurzzeitvariation (STV) ........................................16

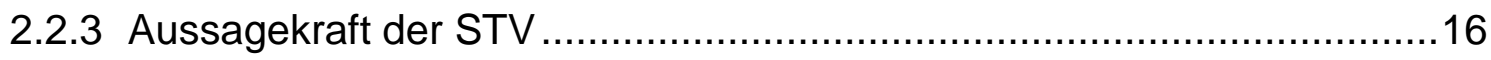

2.3 Das fetale Elektrokardiogramm (EKG) ……....................................

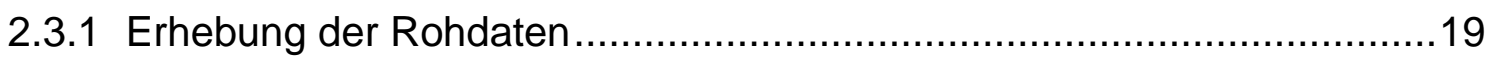

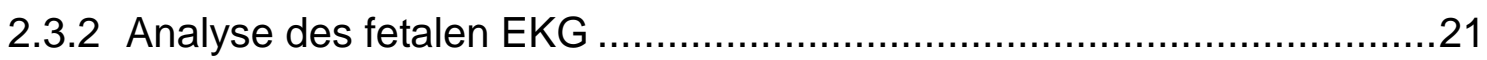

2.4 Phasengleichgerichtete Signalmittelung (PRSA) …..........................22

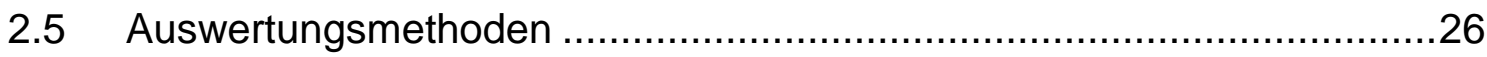

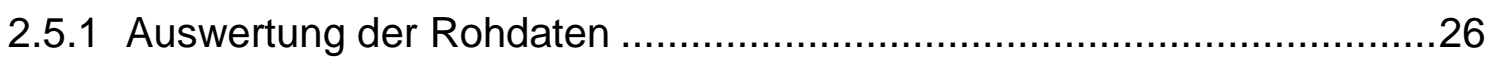

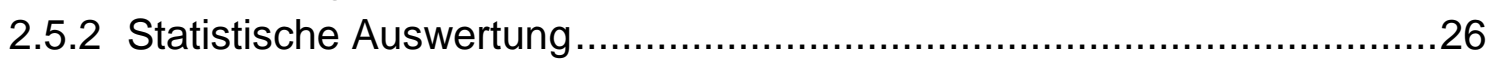

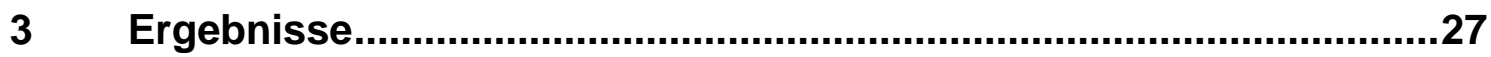

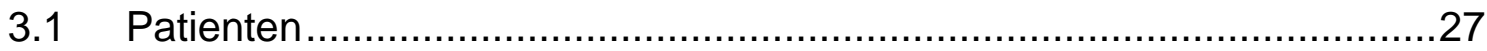

3.2 Statistischer Vergleich der Mittelwerte zu unterschiedlichen Messzeitpunkten mittels T-Test für gepaarte Stichproben ......................29

3.2.1 Übersicht der Mittelwertveränderungen vor und nach

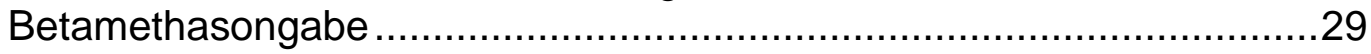

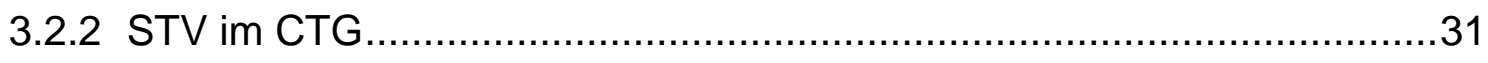

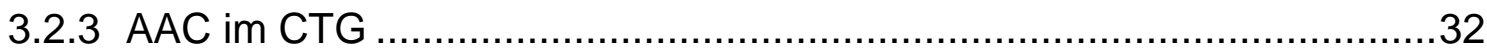

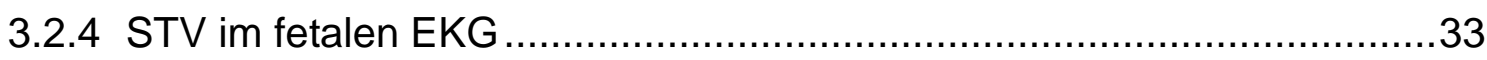

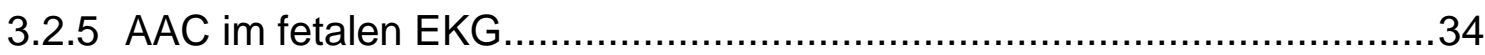

3.2.6 Darstellung der Veränderung der STV und AAC im zeitlichen Verlauf

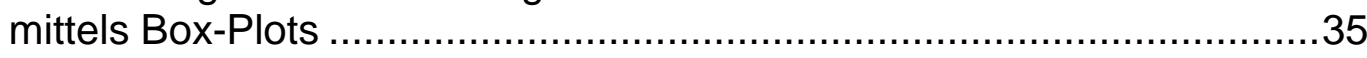

3.3 Korrelation der Messwerte der Parameter vor und nach Steroidgabe ....36

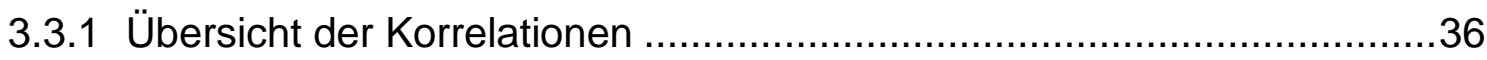

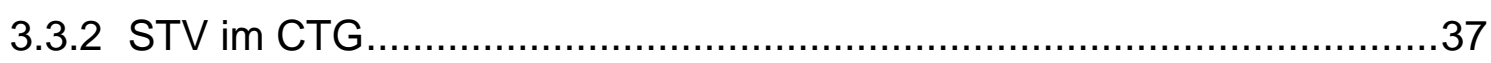

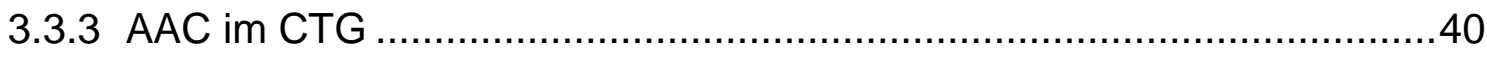

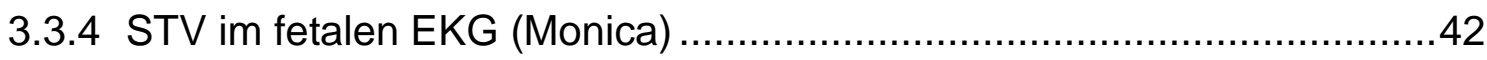


3.3.5 AAC im fetalen EKG (Monica).

3.4 Korrelation der Parameter STV und AAC zu den vier

Messzeitpunkten, Vergleich der Messmethoden... 46

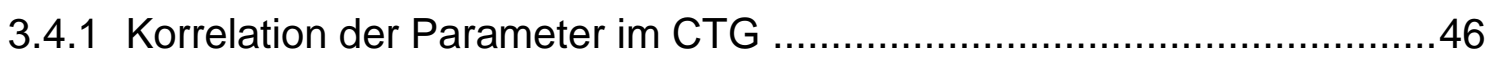

3.4.2 Korrelation der Parameter im fetalen EKG..........................................

3.5 Korrelation der Parameter zwischen den beiden unterschiedlichen Geräten

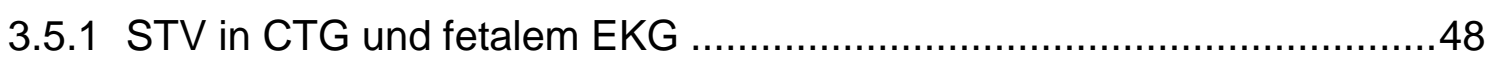

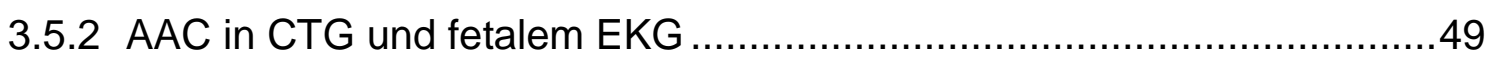

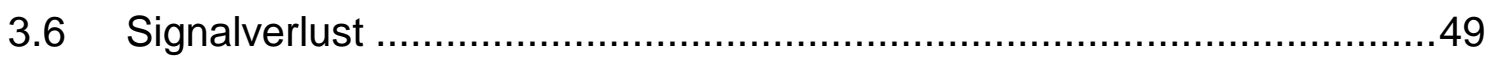

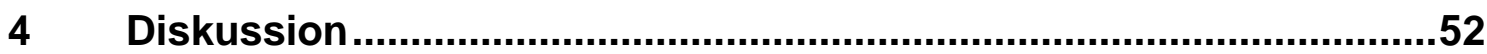

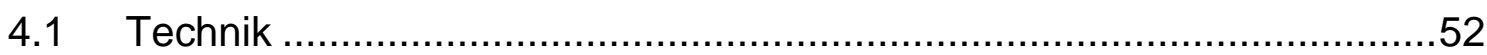

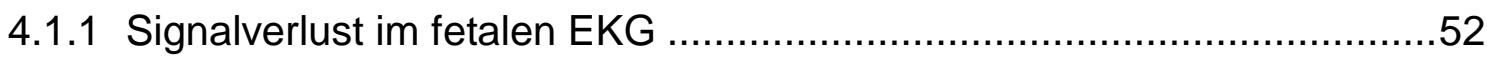

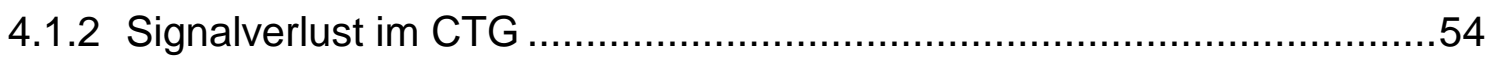

4.1.3 Bedeutung der Signalverluste für die Studie ......................................54

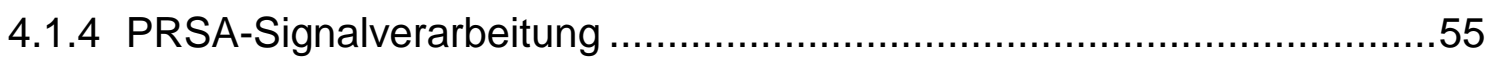

4.2 Physiologie der fetalen Herzfrequenzvariabilität ................................55

4.2.1 Einfluss der antenatalen Kortikosteroidgabe zur Lungenreifeinduktion auf die fetale Herzfrequenzvariabilität ..............................................60

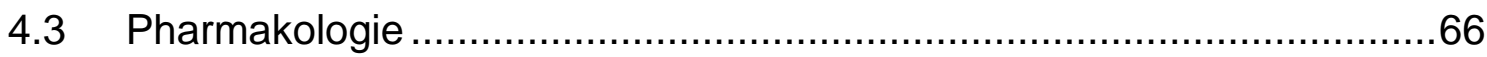

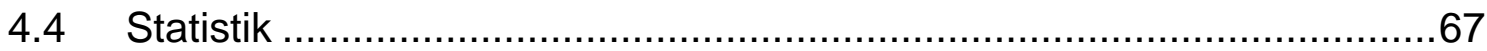

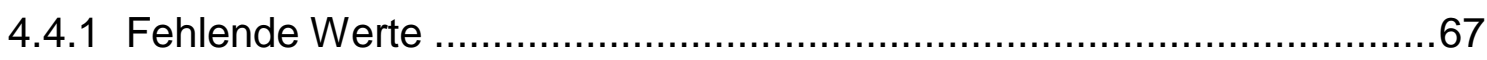

4.4.2 Berücksichtigung fehlender Werte im T-Test und den Korrelationen ......68

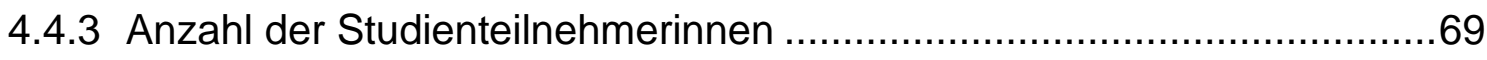

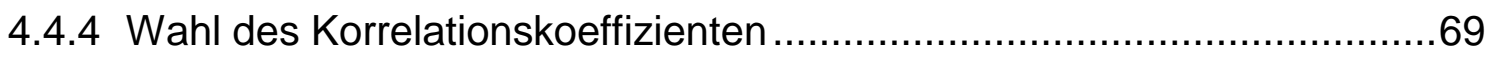

$5 \quad$ Zusammenfassung und Ausblick ......................................................72

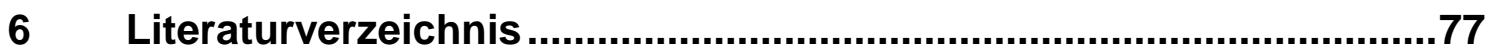

$7 \quad$ Abbildungsverzeichnis......................................................................94

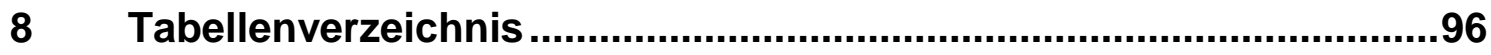

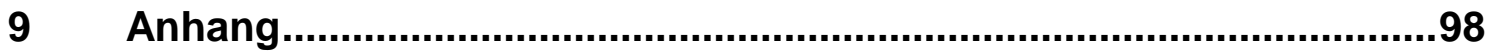

9.1 Studienlage zur iatrogenen Induktion der Lungenreifung mit

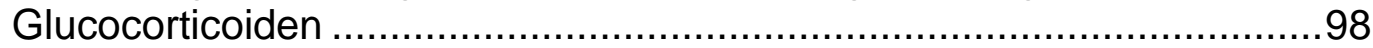

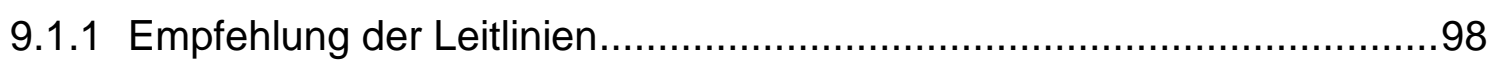

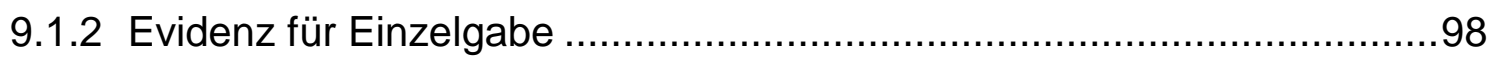

9.1.3 Vergleich von Betamethason und Dexamethason ...............................100

9.1.4 Auswirkung der antenatalen Steroidgabe auf den Feten .....................100 
9.1.5 Auswirkung der antenatalen Steroidgabe auf das Neugeborene 102

9.1.6 Auswirkung der antenatalen Steroidgabe auf die Langzeitentwicklung des Kindes

9.1.7 Kortikosteroideinfluss auf den mütterlichen Organismus 105

9.1.8 Wiederholte Glucocorticoidgabe: Evidenz und Auswirkungen 105

9.2 Die fetale Lunge 109

9.2.1 Entwicklung der fetalen Lunge 109

9.2.2 Physiologische Lungenreifung und Bedeutung der Glucocorticoide .....112

10 Danksagung 115

Erklärung 116 


\section{Abkürzungsverzeichnis}

AAC

Abb.

ACOG

$\mathrm{aM}$

AWMF

BPD

BQS

$\mathrm{Cl}$

CP

CPAP

CTG

(C)PVL

DC

EKG

FHF

h

HF

IUGR

IVH

LTV
Mittlere Akzelerationskapazität

Abbildung

American Congress of Obstetricians and Gynecologists

arithmetisches Mittel

Arbeitsgemeinschaft der Wissenschaftlichen Medizinischen Fachgesellschaften e.V.

Bronchopulmonale Dysplasie

Institut für Qualität \& Patientensicherheit GmbH

confidence interval (engl. Konfidenzintervall)

Cerebralparese

assistierte Beatmung (engl.,continuous positive airway

pressure)

Kardiotokographie

(cystische) periventrikuläre Leukomalazie

Dezelerationskapazität

Elektrokardiogramm

fetale Herzfrequenz

Stunde

„high frequency“ (engl., Beschreibung der Herzfrequenzvariabilität durch Berechnung mittels Spektralanalyse)

intrauterine growth restriction (engl., intrauterine Wachstumsrestriktion)

Intraventrikuläre Hirnblutung

Long term variation (engl. Langzeitvariation) 


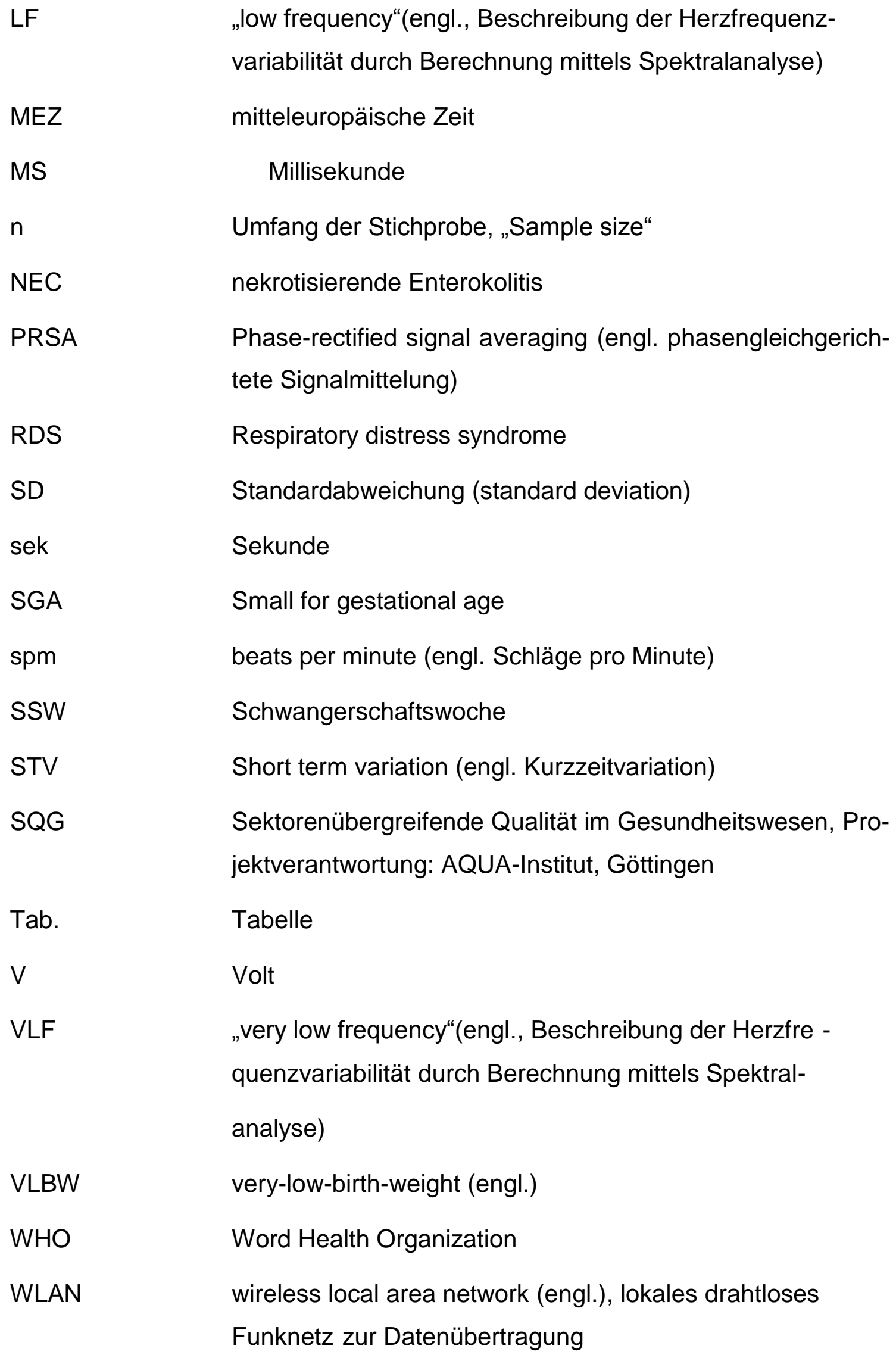




\section{$1 \quad$ Einleitung}

Frühgeburt wird von der Weltgesundheitsorganisation (WHO) als Geburt vor der vollendeten 37. Schwangerschaftswoche bzw. vor weniger als 259 Tagen seit der letzten Menstruation definiert (vgl. Blencowe et al 2012). Der Anteil der Frühgeborenen an den lebendgeborenen Kindern betrug in Deutschland 2008 und 2013 stets ca. 8,7\%, in den USA im Jahr 2010 im Vergleich dazu 11,9\% (vgl. BQS Bundesauswertung Leistungsbereich Geburtshilfe 2008, vgl. SQG Bundesauswertung Leistungsbereich Geburtshilfe 2013, vgl. Martin et al 2012). Im Jahr 2013 trugen die Kinder, die vor der vollendeten 37. Schwangerschaftswoche geboren wurden $76,3 \%$ zur perinatalen Mortalität insgesamt bei (vgl. SQG Bundesauswertung Leistungsbereich Geburtshilfe 2013). Dank der verbesserten interdisziplinären Versorgung der Frühgeborenen und erhöhten Überlebenschancen insbesondere der sehr kleinen Frühgeborenen ist insgesamt eine Abnahme der perinatalen Gesamtmortalität festzustellen (vgl. Schneider H. et al 2011). Im Jahr 2013 lag die perinatale Mortalität insgesamt bei 0,48\% aller Geburten (vgl. SQG Bundesauswertung Leistungsbereich Geburtshilfe 2013).

Die häufigsten Ursachen der Frühgeburt sind Infektionen und Störungen der Plazentation, des Weiteren uterine oder fetale Fehlbildungen. Diese Pathologien können zu vorzeitigen Wehen, vorzeitigem Blasensprung oder aber zur iatrogen indizierten Beendigung der Schwangerschaft führen. In der Diagnostik ist es von zentraler Bedeutung, die Ursache der drohenden Frühgeburt zu identifizieren, um eine eventuelle Therapie beginnen zu können, den Fortschritt der Frühgeburtsbestrebungen zu bestimmen sowie den fetalen Zustand in utero zu beurteilen. Nach Letztgenanntem entscheidet sich das weitere Vorgehen im Sinne einer möglichen Fortsetzung der Schwangerschaft oder aber die Indikation zu einer vorzeitigen Beendigung.

Frühgeborene Kinder haben mit verschiedenen neonatalen Morbiditäten zu kämpfen, wie beispielsweise dem respiratorischen Distress- Syndrom (RDS), intraventrikuläre Hirnblutungen (IVH) und der nekrotisierenden Enterokolitis (NEC) (vgl. Schneider H. et al 2011, vgl. Schleußner 2013). 
Es ist somit von größter Bedeutung in der Geburtshilfe, auf dem Weg zur Indikation für eine vorzeitige Entbindung dem Geburtshelfer präzise und verlässliche diagnostische Werkzeuge an die Hand zu geben, welche inn darin unterstützen sollen, den optimalen Zeitpunkt zur Entbindung zu bestimmen und somit für das Neugeborene bestmögliche, gesundheitliche Bedingungen zu erzielen. Zur Überwachung und Beurteilung der fetalen Situation in utero stehen dem Geburtshelfer verschiedene Mittel zur Verfügung, wie beispielweise die Beurteilung vaskulärer Flussprofile mittels Dopplersonographie, die Beurteilung der fetalen Herzfrequenz und ihrer Variation mittels Kardiotokographie (CTG) und Elektrokardiographie, sowie die Beschreibung des biophysikalischen Profils des Feten. In der Elektrokardiographie kann im Unterschied zur Kardiotokographie die Analyse der fetalen Herzfrequenz anhand der Abstände der R-Zacken konsekutiver QRS Komplexe im EKG (RR- Abstände) von Herzschlag zu Herzschlag erfolgen (beat-to-beat). Die Herzfrequenzvariation ist sowohl in der Erwachsenenmedizin als auch in der Geburtshilfe von zentraler Bedeutung um die Kapazität des autonomen Nervensystems zu evaluieren. Für den Erwachsenen beispielsweise im Hinblick auf die Mortalität nach Myokardinfarkt, für den Feten in Bezug auf sein Wohlbefinden in utero.

Zu den möglichen Maßnahmen bei drohender Frühgeburt zählt neben der Tokolyse und einer antibiotischen Therapie, die Gabe von Glucocorticoiden zwischen der 24. und der 34.Schwangerschaftswoche. Sie dienen der Förderung der Lungenreifung, wodurch schwere pulmonale Komplikationen des Frühgeborenen wie das RDS aber auch das Auftreten von IVH und NEC signifikant reduziert werden können (vgl. AWMF-Leitlinien der deutschen Gesellschaft für Gynäkologie und Geburtshilfe: Antenatale Kortikosteroide zur Lungenreifung, Stand Januar 2014, vgl. Royal College of Obstetricians and Gynaecologists Green-Top Guideline Nr.7: Antenatal Corticosteroids to reduce neonatal morbidity and mortality, Stand Dezember 2014).

Der fetale Organismus und dadurch auch die zur Überwachung in utero verwendeten Parameter wie beispielsweise die Kurzzeitvariation (STV), werden durch die Kortikosteroidgabe jedoch vorübergehend verändert. Es ist demnach von hoher Wichtigkeit, die Auswirkungen der Kortikosteroidtherapie auf die zur 
Evaluation des fetalen Wohlbefindens verwendeten Parameter sehr genau zu kennen und somit korrekt $z u$ interpretieren und sie nicht als Ausdruck fetalen Stresses fehlzudeuten (vgl. Rotmensch et al 2005).

In mehreren Studien wurde bereits der Einfluss der Betamethason-, sowie Dexamethasongabe auf die STV im computerisierten CTG untersucht (vgl. Derks et al 1995, vgl. Senat et al 1998, vgl. Rotmensch et al 1999a, vgl. Rotmensch et al 2005, vgl. Brownfoot et al 2008, vgl. Subtil et al 2003, vgl. Lunshof et al 2005, vgl. Koenen et al 2005, vgl. Mulder et al 2009). Im Abschnitt 4.2.1. „Einfluss der antenatalen Kortikosteroidgabe zur Lungenreifeinduktion auf die fetale Herzfrequenzvariabilität" der vorliegenden Arbeit werden die Ergebnisse dieser Studien ausführlich besprochen.

Darüber hinaus existiert ein neuerer Parameter zur Beschreibung des fetalen autonomen Nervensystems, die mittlere Akzelerationskapazität (AAC), welche durch die Analysemethode der phasengleichgerichteten Signalmittelung (PRSA) entwickelt wurde (vgl. Huhn et al 2011, vgl. Lobmaier et al 2012). Lobmaier und Huhn konnten bereits für IUGR-Feten zeigen, dass die AAC als Überwachungsparameter der fetalen Herzfrequenz im CTG eine genauere und zuverlässigere Unterscheidung zwischen normalgewichtigen Feten und IUGRFeten ermöglicht als die STV (vgl. Lobmaier et al 2012). Graatsma et al konnten für die Analyse der fetalen Herzfrequenz mittels fetalem EKG zeigen, dass die AAC besser geeignet ist, um zwischen Kontrollen und small-for-gestational-age (SGA)- Feten zu differenzieren, als die STV (vgl. Graatsma et al 2012).

In der vorliegenden Arbeit soll das Verhalten des Parameters AAC im Vergleich zum Verhalten der STV unter Betamethasoneinfluss zur Lungenreifeinduktion bei drohender Frühgeburt untersucht werden.

Die fetale Herzaktivität wird hierfür parallel mittels CTG und fetalem EKG registriert, auch die beiden Messmethoden werden miteinander verglichen. 


\section{Methodik}

\subsection{Patientenrekrutierung}

In einer prospektiven Studie wurden von August 2010 bis Mai 2012 bei 28 Patientinnen der Frauenklinik des Klinikum rechts der Isar, welche Betamethason (Celestan $\AA$ solubile $2 \times 12 \mathrm{mg}$ im Abstand von $24 \mathrm{~h}$ ) zur Lungenreifebehandlung bei drohender Frühgeburt erhielten, Aufzeichnungen der fetalen Herzfrequenz mittels computerisiertem CTG sowie zeitgleich mittels fetalem EKG durchgeführt.

Die Aufzeichnungen dauerten mindestens 40 Minuten und erfolgten vor der Kortikosteroidgabe (Zeitpunkt 0 Stunden), sowie 24 Stunden, 48 Stunden und 96 Stunden nach der ersten Gabe des Medikaments.

Die wiederholten Messungen wurden für die jeweilige Patientin stets zur selben Tageszeit durchgeführt. Im Median lag der Messzeitpunkt am Nachmittag um ca.17:00 Uhr.

Einschlusskriterien:

- Alter über 18 Jahre

- Schwangerschaftsalter zwischen 24 und 34 Schwangerschaftswochen

- Indikation zur Lungenreifeinduktion gestellt

- Einlingsschwangerschaften

- Einverständniserklärung der Patientinnen

Auschlusskriterien:

- Unmittelbar drohende oder notwendige Entbindung

- Mehrlingsschwangerschaften

- Bekannte fetale Herzrhythmusstörungen

- Vorbestehende maternale Medikation mit Kortison

- Kontraindikationen für Betamethasongabe 
Die CTG Aufzeichnungen erfolgten mit dem Gerät Sonicaid Team Care, die computerisierte Analyse durch die Software Sonicaid Fetal Care (Gerät- und Softwarehersteller: HNE Huntleigh Nesbit Evans Healthcare GmbH, Cardiff, UK).

Das fetale Elektrokardiogramm wurde mit dem Gerät AN24 ${ }^{\mathrm{TM}}$ der Firma Monica Healthcare Ltd. Nottingham, UK aufgezeichnet. Es wurden fünf nicht-invasive, selbstklebende Elektroden in standardisierter Position auf den Bauch der Mutter aufgebracht.

\subsection{Das Kardiotokogramm (CTG)}

Grundprinzip der Kardiotokographie ist die Berechnung der fetalen Herzfrequenz mittels Dopplersonographie: Beim hierbei angewandten Puls- Echo- Verfahren trifft das von den Piezoelektrischen Elementen des Schallkopfs erzeugte Ultraschallsignal auf das sich in Bewegung befindliche, fetale Herz. Dies führt zu Frequenzverschiebungen der reflektierten Signale, die wiederum vom Schallkopf erfasst werden. Die interne Verarbeitung der eintreffenden Signale kann im Gerät zum Beispiel durch Triggerverfahren erfolgen, welche in neueren Geräten durch den Mechanismus der Autokorrelation ergänzt werden. Durch diesen Mechanismus wird zwar die Verlässlichkeit der Ergebnisse erhöht, jedoch ist eine Analyse der fetalen Herzaktivität auf einer Schlag- zuSchlag- Ebene nicht möglich (vgl. Gnirs J., Schneider K.T.M. 2011, vgl. Peters et al 2001).

In der computerisierten CTG- Analyse wird die Herzfrequenzkurve des Kindes parallel zu ihrer Registrierung auf dem Computer graphisch dargestellt und mittels einer Computersoftware analysiert. Die computerisierte Auswertung der CTG- Aufzeichnungen kann medizinisches Personal in der Bewertung der CTG Ergebnisse unterstützen. Es verringert die Intra- und Interobserver- Variabilität in der Beurteilung der CTG Aufzeichnung, wodurch einheitlichere Ergebnisse erzielt werden können, dies gilt besonders für die Interobserver- Variabilität (vgl. Keith et al 1995, vgl. Devoe et al 2000). 
In dieser Studie wurde das CTG Sonicaid Team Care des Klinikums rechts der Isar mit der Analyse Software Sonicaid Fetal Care (Gerät- und Softwarehersteller: Firma HNE Huntleigh Nesbit Evans Healthcare GmbH, Cardiff, UK; ehemals Oxford Instruments) die Weiterentwicklung der Software Sonicaid System 8002 (Oxford Instruments Medical Ltd, Surrey, UK) verwendet. Sie basiert auf einer Datenbank, welche 73.802 Kurvenverläufe in Korrelation mit dem späteren Outcome der Kinder erfasst hat (vgl. Pardey et al 2002). Diese dienen als Referenzkollektiv in der Analyse der Software: die Parameter der Dawes/Redman Analyse, deren Berechnung im Folgenden näher ausgeführt werden, werden von der Software im Hinblick auf inre Übereinstimmung mit diesem Kollektiv untersucht. Abbildung 1 zeigt eine typische Aufzeichnung der fetalen Herzaktivität mit dem in der Studie verwendeten CTG (Sonicaid Team Care), sowie die Analyseergebnisse durch die Software. 


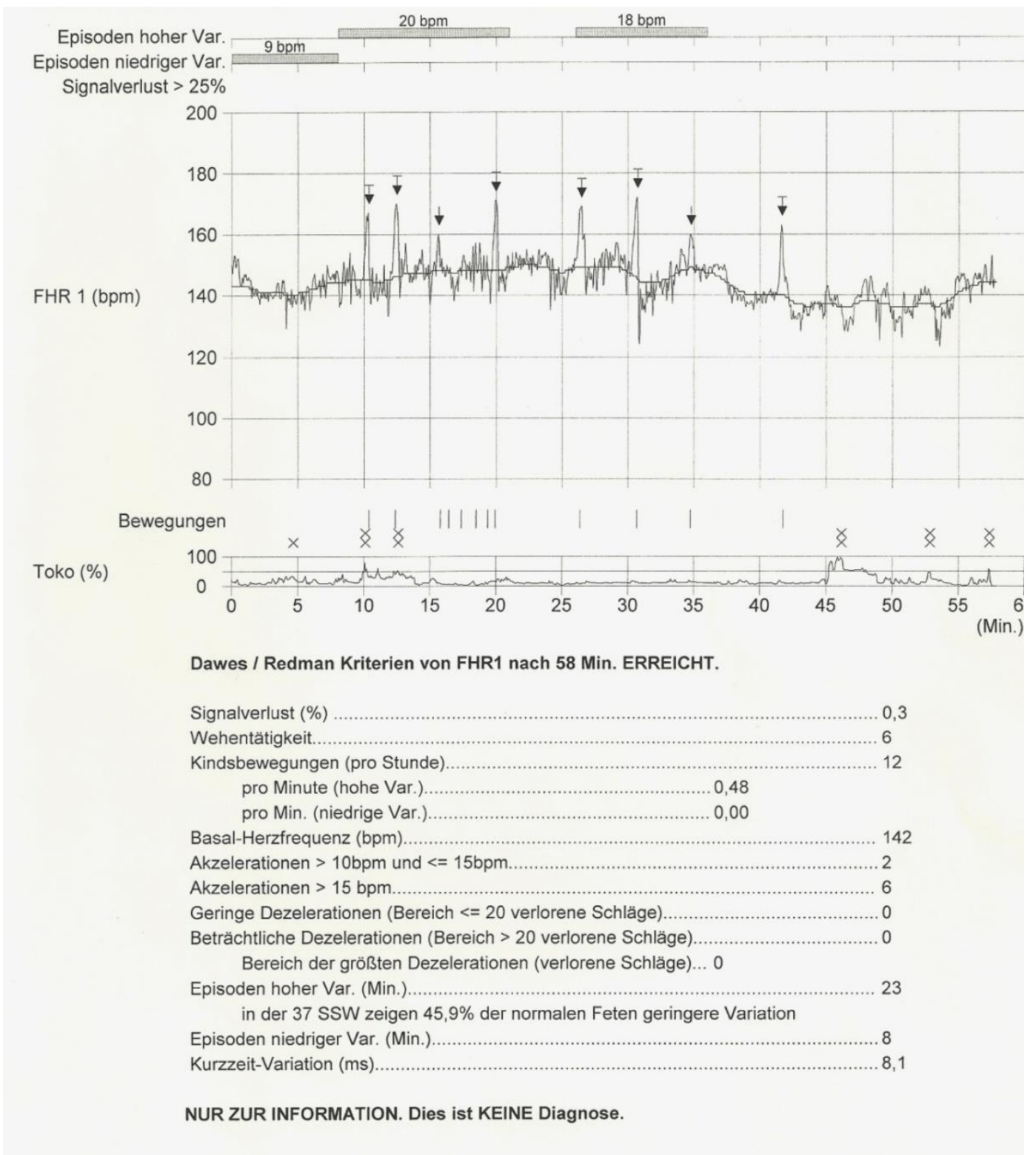

Abb.1: Exemplarische Aufzeichnung der fetalen Herzaktivität mit dem in der Studie verwendeten CTG (Sonicaid Team Care, Klinikum rechts der Isar); oberer Teil der Abbildung: graphische Darstellung der fetalen Herzaktivität sowie der uterinen Kontraktionen, unterer Teil der Abbildung: Analyseergebnisse der Software (Sonicaid Fetal Care).

Die Parameter werden zur Überprüfung der fetalen Herzaktion mittels der Dawes/Redman Kriterien verwendet, deren Erreichen Anhalt für einen guten Gesundheitszustand des Feten geben können. Diese Kriterien sind empirisch be- 
gründet und basieren auf den Erhebungen von Prof. Geoffrey S. Dawes und Prof. Christopher Redman.

Für die Analyse teilt die Software zunächst eine Minute der kindlichen Herzaktion im CTG in 16 je 3.75 Sekunden (sek) dauernde Epochen ein. Für jede Epoche berechnet sie die durchschnittliche fetale Herzfrequenz in Schlägen pro Minute (spm) und das Pulsintervall (in ms), wobei dies der Zeit zwischen zwei Herzschlägen entspricht. Für die Analyse wird das mittlere Pulsintervall aller Epochen verwendet.

Bei einer Herzfrequenz von 120 spm würden demnach 7,5 Schläge pro Epoche erfolgen, wonach das Pulsintervall (3,75 sek: 7,5 Schläge) 0,5 sek bzw. 500ms lang wäre.

Die erste Analyse während einer Aufzeichnung erfolgt nach 10 Minuten, danach alle zwei Minuten (vgl. Pardey et al 2002).

\subsubsection{Berechnung der Parameter der Dawes-Redman Analyse}

Zunächst erfolgt die Festlegung der Basallinie durch Ermittlung der Grundfrequenz (Einheit spm). Relativ zu ihr werden Akzelerationen bzw. Dezelerationen der fetalen Herzfrequenz angegeben. Dabei gelten Frequenzen, die mindestens 15 spm schneller als die Frequenz der Basallinie sind und mindestens 15 Sekunden andauern als Akzelerationen, bzw. vor der 32. SSW Frequenzen über 10 spm mit einer Dauer von mehr als 10 Sekunden. Beim Anhalten von Akzelerationen länger als 10 Minuten spricht man von einer Veränderung der Basallinie. Eine Dezeleration ist definiert als ein Absinken der Frequenz unter die Basallinie um mindestens 15 spm für über 15 Sekunden. Die Grundlinie (Einheit spm) ist die mittlere, beibehaltene fetale Herzfrequenz über mindestens $10 \mathrm{Mi}$ nuten in Abwesenheit von Akzelerationen oder Dezelerationen. Ihr Normbereich liegt zwischen 110-160 Spm. Im Bereich der fetalen Unreife liegt die durchschnittliche fetale Herzfrequenz im oberen Streubereich. (vgl. Gnirs J, Schneider K.T.M 2011, vgl. AWMF-Leitlinien der deutschen Gesellschaft für Gynäkologie und Geburtshilfe 08/2013: Anwendung des CTG während Schwangerschaft und Geburt, Stand Dezember 2014). 
In der späten Schwangerschaft lässt sich ein deutlicher Zusammenhang zwischen kindlichen Bewegungen und reaktiven Akzelerationen in der Herzfrequenz nachweisen (vgl. Pillai et al 1990a). Die in dieser Phase der Schwangerschaft teilweise recht lebhaften Bewegungen führen zu schnellen zyklischen Variationen in der Herzfrequenz, dieses Phänomen tritt besonders in den frühen Abendstunden auf (vgl. Dawes et al 1982).

Aus den zwischen drei- bis fünfmal pro Minute auftretenden Fluktuationen der fetalen Grundfrequenz, wird die sogenannte Variabilität oder Bandbreite der fetalen Herzfrequenz bestimmt. Sie berechnet sich aus der Differenz (in spm) der höchsten bzw. tiefsten Fluktuation der fetalen Grundfrequenz in der auffälligsten Minute innerhalb eines dreißigminütigen Registrierungsintervalls (vgl. AWMF-Leitlinien der deutschen Gesellschaft für Gynäkologie und Geburtshilfe 08/2013: Anwendung des CTG während Schwangerschaft und Geburt, Stand Dezember 2014). Die Variation der fetalen Herzfrequenz ist ein wesentlicher Bestandteil der Analyse. Sie kann auf verschiedene Weisen beschrieben werden, die entsprechenden Parameter sind die Langzeitvariation (LTV) und die Kurzzeitvariation (STV).

Für die Berechnung der LTV wird zunächst der sogenannte Minutenwert ermittelt. Dieser wird relativ zur Basallinie bestimmt. Er errechnet sich als Differenz zwischen dem maximalen und minimalen Wert der Pulsintervalle innerhalb einer Minute und wird in ms angegeben (vgl. Dawes et al 1991).

Der Mittelwert aller aufeinander folgenden Minutenwerte einer Aufzeichnung, der sogenannte mittlere Minutenwert, entspricht der LTV (vgl. Pardey et al 2002). Mit zunehmendem Gestationsalter steigt der mittlere Minutenwert leicht an. Der Normalwert liegt bei 50ms oder entsprechend $17 \mathrm{Spm}$ (vgl. Street et al 1991).

Eine Episode hoher Variation kennzeichnet sich durch einen Minutenwert in mindestens fünf von sechs aufeinanderfolgenden Minuten, der $32 \mathrm{~ms}$ oder mehr beträgt. Als Episode niedriger Variation wird ab einem Minutenwert von kleiner oder gleich $30 \mathrm{~ms}$ in wenigstens fünf von sechs konsekutiven Minuten gesprochen (vgl. Pardey et al 2002). 


\subsubsection{Berechnung der Kurzzeitvariation (STV)}

Ein wichtiger Parameter zur Analyse der fetalen Herzfrequenz ist die Kurzzeitvariation (STV). Da es mit dem CTG auf Grund der Autokorrelation nicht möglich ist Variationen von Schlag zu Schlag zu bestimmen, errechnet die Software sie aus der mittleren Variation im Pulsintervall von Epoche zu Epoche.

Dies geschieht, in dem stets die Differenz zwischen dem Mittelwert der Pulsintervalle einer Epoche zum Mittelwert der darauffolgenden Epoche gebildet wird.

Diese Differenzen werden nun über eine Minute und der entstandene Wert schließlich über die Dauer der Aufzeichnung gemittelt (vgl. Dawes et al 1991, vgl. Pardey et al 2002).

Die vorgenommene Mittelung in zwei Schritten, das heißt zunächst der Differenzen der Mittleren-Pulsintervalle innerhalb einer Minute und anschließend über die gesamte Aufzeichnung, ist wichtig, um dem Problem des Signalverlustes in Epochen während Episoden hoher Frequenzvariation gerecht zu werden. Tritt während diesen Episoden ein Signalverlust auf, führt dies zu einer geringere Anzahl gültiger Epochen in diesem Zeitraum als während Episoden niedriger Variation, in denen der Signalverlust kleiner ist. Durch die Mittelung in zwei Schritten werden die Werte der einzelnen Minuten stärker gewichtet als bei einer Mittelung aller Epochen in einem einzigen Schritt, wodurch diesem Problem entgegen gewirkt wird (vgl. Pardey et al 2002).

Minuten mit Signalverlusten ab 50\% oder Anteil an Dezelerationen werden bei der Berechnung der LTV und STV und der Bestimmung von Episoden hoher und niedriger Variation nicht mit einbezogen. Die Herzfrequenzvariation oder variabilität hängt auch vom Verhaltenszustand des Fetus ab (vgl. Pardey et al 2002).

\subsubsection{Aussagekraft der STV}

Die Kurzzeitvariation ist ein umfangreich untersuchter Parameter der computerisierten CTG Analyse (vgl. Galazios et al 2010). Er korreliert eng mit dem fetalen Outcome (vgl. Schneider K.T.M., Gnirs J. 2011). Dawes et al konnten zeigen, dass die Wahrscheinlichkeit für eine fetale Azidose (mit einem pH der Arte- 
ria umbilicalis $<7.12$ ) oder einen intrauterinen Fruchttod bei $38 \%$ lag, wenn die STV $<2.6 \mathrm{~ms}$ betrug, hingegen $9 \%$ wenn sie zwischen $2.6-3 \mathrm{~ms}$ betrug (vgl. Dawes et al 1992a). Street et al beschrieben, dass mit einer STV $<=2.5 \mathrm{~ms}$ die terminalen Feten identifiziert werden konnten und zeigten eine hohe Korrelation zwischen LTV und STV $(r=0.9)$ auf (vgl. Street et al 1991). Ein Wert $<=3 \mathrm{~ms}$ findet sich bei präterminalem Zustand des Feten (vgl. Dawes et al 1992a, vgl. Schneider K.T.M., Gnirs J. 2011). Bei fetalem Wohlbefinden ist eine STV von $>=6 \mathrm{~ms}$ zu erwarten, bei chronischer Hypoxie eine STV $<=4 \mathrm{~ms}$ (vgl. Schneider K.T.M., Gnirs J. 2011).

\subsection{Das fetale Elektrokardiogramm (EKG)}

Eine weitere Möglichkeit zur intrauterinen Überwachung des Fetus ist das fetale EKG. Es registriert die elektrische Aktivität des fetalen Herzens, abgeleitet über die mütterliche Bauchdecke und kann ab der 20. Schwangerschaftswoche genutzt werden. Der Einsatz des fetalen EKG ist grundsätzlich auch bei Mehrlingsschwangerschaften möglich (vgl. Peters et al 2001).

Eine Analyse der fetalen Herzfrequenz von Schlag zu Schlag (beat- to- beat) ist mit dem fetalen EKG, im Gegensatz zum CTG, bei dem die Technik der Autokorrelation dies verhindert, möglich. Die fetale Herzratenvariation kann so mit dem EKG präziser beurteilt werden (vgl. Peters et al 2001).

Abbildung 2 zeigt die Aufzeichnung eines fetalen, abdominellen EKGs mit dem Gerät Monica AN24 ${ }^{\mathrm{TM}}$ abgebildet mit der Software Monica DK. In Abbildung 3 lässt sich die Morphologie des EKG in der Software Monica DK, bspw. der QRS-Komplex, erkennen. 


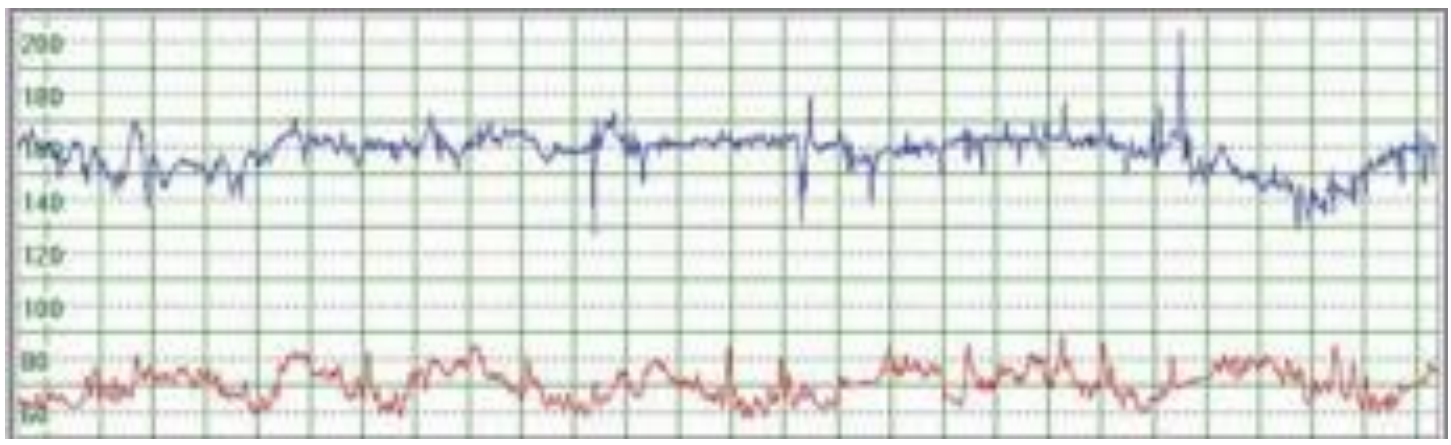

Abb. 2: Darstellung der fetalen (oben) und mütterlichen Herzfrequenz (unten) durch die Software Monica DK im Anschluss an die Aufzeichnung.

(Quelle: http://www.monicahealthcare.com/products/Monica-DK-Product-Sheet.pdf, Stand Dezember 2014).

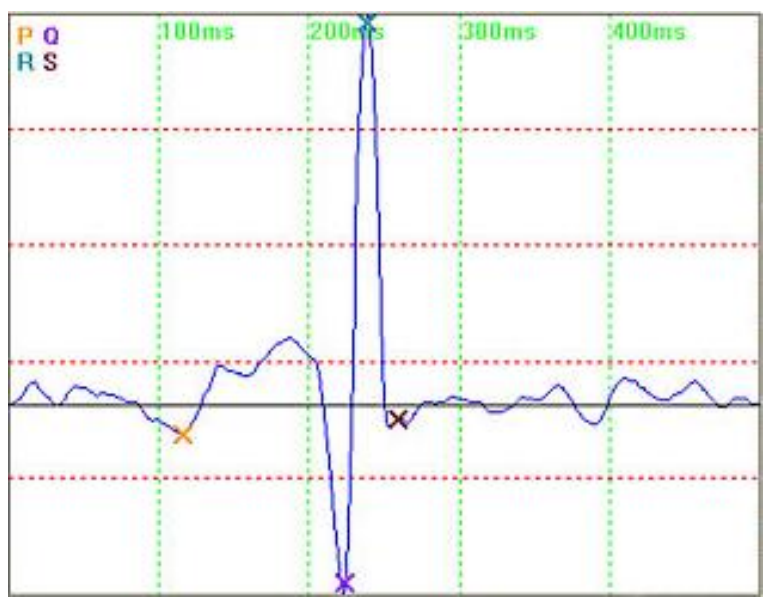

Abb.3: EKG-Kurve, abgebildet durch die Software Monica DK

(Quelle: http://www.monicahealthcare.com/products/Monica-DK.php, Stand Dezember 2014).

Die Akzeptanz des Gerätes auf Patientenseite ist hoch (vgl. Philippe et al 2012, Reinhard et al 2008). Insbesondere für Langzeitmessungen ist dies ein entscheidender Vorteil gegenüber andern Techniken wie dem Doppler oder dem CTG, da diese ambulant und ohne größere Einschränkungen für die Patientin erfolgen können (vgl. Peters et al 2001, vgl. Piéri et al 2001).

Obwohl die Technik der fetalen Elektrokardiographie schon länger existiert, hat sie sich bislang dennoch nicht als Standard im klinischen Alltag bewährt. Dies liegt insbesondere daran, dass auf Grund des schlechten Signal-zu-RauschVerhältnisses die Signalqualität stark variieren kann. Damit ist die Aufzeichnungsqualität der Rohdaten nicht stets gleichbleibend garantiert (vgl. Peters et al 2001, Piéri et al 2001). Verschieden Faktoren tragen zu dem schlechten Sig- 
nal-zu-Rausch- Verhältnis bei: Neben dem Signalrauschen durch andere elektrische Phänomene wie die abdominelle Muskulatur der Mutter, spielen auch die Menge an Fruchtwasser und die, besonders zwischen der 28.-32. Schwangerschaftswoche vermehrte, Vernix caseosa eine wichtige Rolle. Diese isoliert den Fetus elektrisch (vgl. Peters et al 2001, Piéri et al 2001). Peters et al geben die Verlässlichkeit des Messverfahrens mit ca. 60\% innerhalb späterer Schwangerschaftswochen an, für die Dopplersonografie im Vergleich hingegen über 95\% (vgl. Peters et al 2001).

\subsubsection{Erhebung der Rohdaten}

Die elektrische Aktivität des fetalen Herzens kann mittels auf der mütterlichen Haut aufgebrachter Elektroden aufgezeichnet werden. Es werden fünf Elektroden am Bauch der Mutter in standardisierter Weise aufgebracht, in Abbildung 4 ist die Elektrodenpositionierung dargestellt. Zur Verringerung der Hautimpendanz wird die Epidermis davor mit einem leicht dermo-abrasiven Streifen aufgeraut. Die Elektroden sind mit dem tragbaren Gerät über dekonnektierbare Kabel verbunden (s. Abb.4).

In dieser Arbeit wurde das Gerät AN24 ${ }^{\mathrm{TM}}$ der Firma Monica Healthcare Ltd. Nottingham, UK verwendet, welches in Abbildung 5 dargestellt ist. Nach der Datenaufzeichnung wurden die Rohdaten auf den Computer übertragen, zur anschließenden Analyse der Signale wurde die Software Monica DK 1.5b verwendet.

Da über die Elektroden sämtliche elektrische Aktivität im mütterlichen Körper aufgezeichnet wird, erhält der Untersucher neben dem fetalen auch das Signal der mütterlichen Herzaktivität sowie ein Elektrohysterogramm. Das Signal des mütterlichen Herzens liegt in einer Größenordnung von $100 \mu \mathrm{V}$, das fetale Signal bei nur etwa $10 \mu \mathrm{V}$ (vgl. Peters et al 2001).

Das Gerät AN24 ${ }^{\mathrm{TM}}$ gibt dem Untersucher zu Beginn der Aufzeichnung zu erkennen, ob es ein gültiges Signal von Mutter und Kind empfangen kann. Die Platzierung der Elektroden kann daraufhin gegebenenfalls korrigiert werden. Zwischen der 28. und 32 SSW hängt die schlechtere Signalqualität häufig mit 
dem vermehrten Gehalt an Vernix caseosa zusammen. Leider ist trotz dieser vorangehenden Überprüfung ein Signalverlust möglich (vgl. Philippe et al 2012).

Das Signal wird über mehrere Kanäle aufgezeichnet, was die Signalakquirierung verbessert. Die zu erfassende Bandbreite der fetalen Herzfrequenz liegt dabei im Bereich von 100-1000 Hz (vgl. Lobmaier et al 2012). Die Rohdaten werden auf den Computer übertragen, wo sie von der Software weiterverarbeitet werden (vgl. Piéri et al 2001).

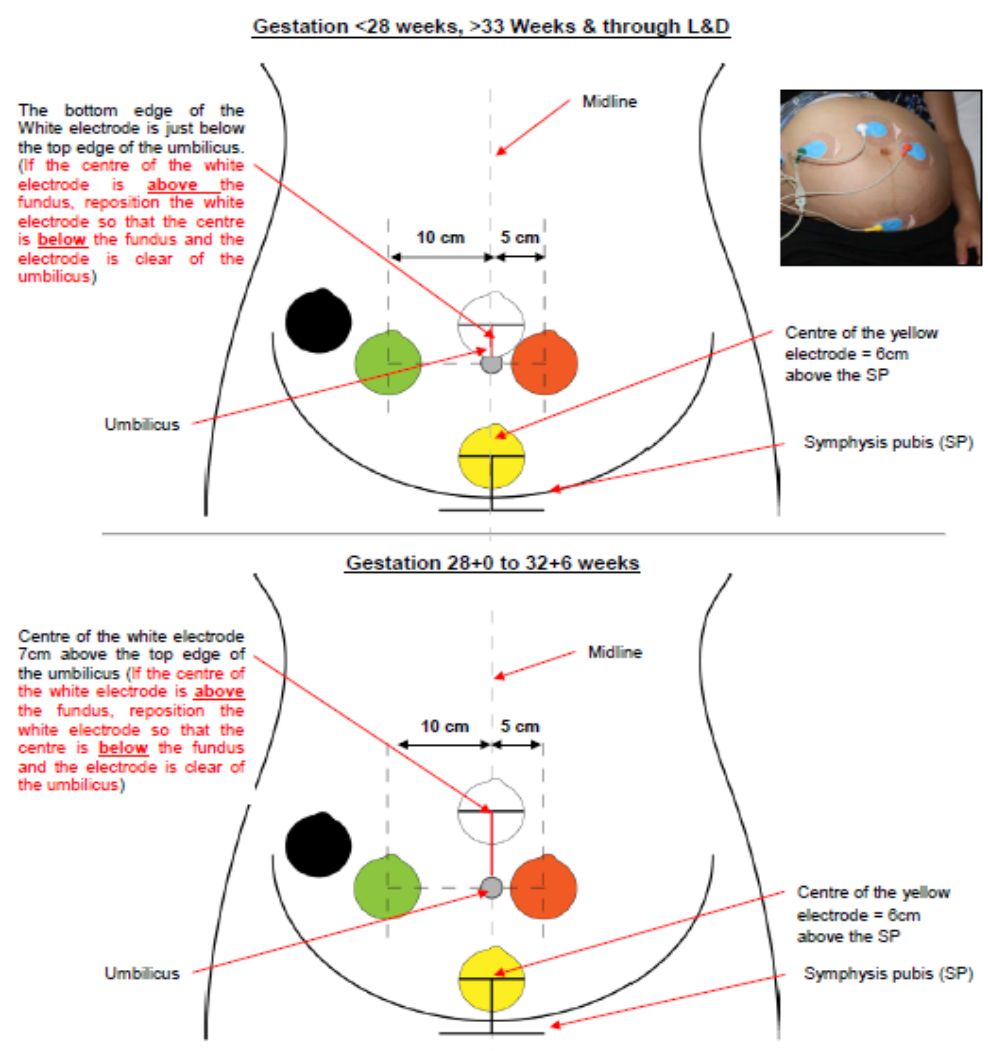

Abb.4: Fetales EKG: Positionierung der Elektroden (Quelle: Monica Healthcare AN24 ${ }^{\mathrm{TM}}$ Reference Operator Manual, Monica Healthcare Ltd. 2007-2009). Oberer Teil der Abbildung: Positionierung der Elektroden im Gestationsalter <28SSW bzw. >33SSW. Beschreibung: Der Unterrand der weißen Elektrode sollte unmittelbar an den Oberrand des maternalen Bauchnabels angrenzen. Hierbei muss jedoch gewährleistet sein, dass sich das Zentrum der Elektrode dabei unterhalb des Fundus befindet. Ist dies nicht der Fall, erfolgt eine tiefere Platzierung der Elektrode. Das Zentrum der gelben Elektrode sollte $6 \mathrm{~cm}$ oberhalb der Symphysis pubica platziert werden. Die Platzierung der grünen und roten Elektrode erfolgt entsprechend den Zentimeterangaben der Abbildung in Bezug auf die weiße Elektrode auf Höhe des Umbilicus. Die schwarze Referenzelektrode wird dorsolateral an der maternalen Bauchdecke platziert. Unterer Teil 
der Abbildung: Positionierung der Elektroden zwischen der 28+0-32+6 SSW. Beschreibung: Das Zentrum der weißen Elektrode sollte nun einen Abstand von $7 \mathrm{~cm}$ zum Oberrand des Umbilicus haben. Auch hier gilt es, die beschriebene Lagebeziehung zum Fundus zu beachten und die Elektrode gegebenenfalls tiefer zu platzieren. Die restlichen Elektroden werden wie in der oberen Abbildung beschrieben platziert.

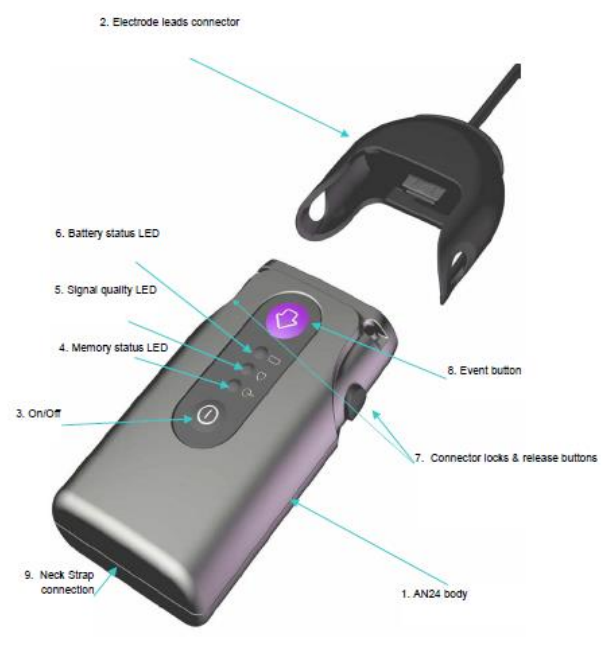

Abb. 5: Monica AN24 ${ }^{\mathrm{TM}}$ Gerät mit dekonnektierbarem Verbindungsstück (schwarz) welches Kabel zu den Elektroden führt (Quelle: Monica Healthcare AN24 ${ }^{\text {TM }}$ Reference Operator Manual, Monica Healthcare Ltd. 2007-2009).

\subsubsection{Analyse des fetalen EKG}

Das Prinzip, nach dem die Software zur Analyse des Elektrokardiogramms arbeitet, wurde von Piéri et al 2001 beschrieben und ist im Folgenden zusammengefasst:

Die Software unterscheidet und trennt fetale und mütterliche EKG- Komplexe und berechnet die entsprechenden Herzraten (vgl. Piéri et al). Dies kann zum Beispiel durch Linearfilterung geschehen. Hierbei wird zunächst der mütterliche QRS- Komplex mittels Korrelationsfilter (engl. "matched filter" vgl. Ohm et al 2005, S.184-187) detektiert, das mütterliche Signal wird anschließend vom gesamten Signal subtrahiert, wodurch das fetale Signal übrig bleibt. Eine andere Möglichkeit ist das sog. "blanking" (s. Piéri et al 2001), wobei das mütterliche Signal unterdrückt wird und schließend die Bestandteile der QRS- Komplexe im fetalen Signal mittels Korrelationsfilter detektiert werden. 
Die fetale Herzrate wird aus den Abständen der R-Zacken konsekutiver QRS Komplexe berechnet, den sog. fetalen RR-Intervallen. Entsprechendes gilt für die mütterliche Herzfrequenz.

Beide EKG- Signale werden in Epochen mit der Dauer von zwei Sekunden unterteilt. Über jede Epoche werden die fetale Herzrate bzw. die mütterliche Herzrate gemittelt. Die erhaltenen Werte dienen der besseren Vergleichbarkeit mit anderen Verfahren beispielsweise mit der Dopplersonographie (vgl. Piéri et al 2001).

Die Berechnung der Kurzzeitvariation (STV) erfolgte für das fetale EKG durch die Software Monica DK 1.5.b der Firma Monica Healthcare Ltd. Nottingham, UK., nach der etablierten Methode nach Dawes und Redman (vgl. Dawes et al 1991, vgl. Graatsma et al 2012).

\subsection{Phasengleichgerichtete Signalmittelung (PRSA)}

Die Technik der PRSA (engl. Phase-rectified signal averaging) dient der Analyse von biologischen Signalen mit periodischem und quasi-periodischem Signalverhalten.

Biologische Signale wie beispielsweise die Herzaktivität weisen zwar einen periodischen Signalcharakter auf, jedoch unterliegen sie als dynamische Systeme stetig Störeinflüssen, welche dieses rein periodische Signalverhalten stören. Dies führt dazu, dass das Signal einen quasi- periodischen Charakter annimmt. Es besteht aus mehreren periodischen Abschnitten, die jedoch desynchronisiert sind. Ihre Länge bestimmt die sogenannte Kohärenzzeit der Quasiperiodizität.

Quasi-periodische Signale können von konventionellen Methoden wie der Spektralanalyse auf Basis der Fourier Transformation nicht umfassend quantifiziert werden.

Durch die Entwicklung der Signalverarbeitungstechnik PRSA, die sog. phasengleichgerichtete Signalmittelung, gelingt es jedoch quasi-periodische Signale zu finden und zu messen, insbesondere auch dann wenn sie durch Nicht- Periodizitäten, Artefakte und Rauschen verdeckt sind. 
Dies geschieht durch die Festlegung von Ankerpunkten im Signal. Durch sie können die Phasen, der den Ankerpunkt umgebenden Signaloszillationen, zunächst an einander angeglichen werden, dies entspricht der sogenannten „Phasengleichrichtung“(vgl. Huhn 2006, vgl. Lobmaier 2012). Anschließend werden die Werte gemittelt. Typischerweise werden etwa die Hälfte aller Punkte eines betrachteten Zeitfensters zu Ankerpunkten (vgl. Bauer et al 2006a).

Durch die Technik der PRSA ist es somit möglich, Akzelerations- und Dezelerationskapazität des Herzens zu evaluieren. Dadurch konnte in einer Kohortenstudie an Postinfarktpatienten für die Dezelerationskapazität gezeigt werden, dass dieser Parameter den Zusammenhang zwischen reduzierter vagaler Aktivität am Myokard nach Infarkt, die folglich reduzierte Herzfrequenzvariabilität und die damit verbundene erhöhte Mortalität des Patienten, genauer vorherzusagen vermag als andere, einschließlich der linksventrikulären Ejektionsfraktion (vgl. Bauer et al 2006b).

Um die Ankerpunkte innerhalb eines Signalabschnitts festzulegen, erfolgt zunächst die Bestimmung einer Filterbedingung, wie im Folgenden gezeigt. Diese kann beispielweise die Zu- oder Abnahme der Herzfrequenz um den definierten Ankerpunkt $\left(X_{i}\right)$ herum sein.

Zunächst wird eine beliebige Anzahl an Schlägen vor und nach $X_{i}$ festgelegt, welche die sogenannte Filterlänge $T$ bilden. In Abb. 6 ist eine Filterlänge $T=5$ dargestellt, mit fünf Herzfrequenzwerten vor und fünf Werten nach $X_{i}$ (grüne und rote Punkte). Mittelt man nun die Werte der Herzfrequenzwerte in $T$ vor und nach $X_{i}$, (in Abb. 6 entspricht dies den schwarzen Balken), kann man daraus den Anstieg bzw. Abfall der Herzfrequenz um $X_{i}$ herum erkennen und somit entscheiden ob $X_{i}$ die geforderte Filterbedingung erfült und damit zum Ankerpunkt wird oder nicht (vgl. Lobmaier et al 2012). 


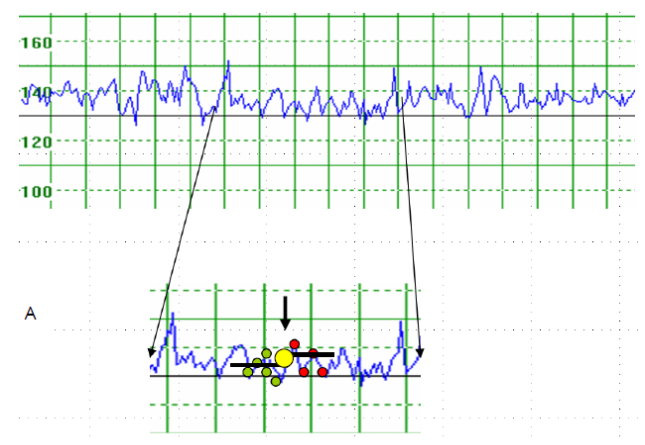

Abb.6 Überprüfung der Filterbedingung um einen möglichen Ankerpunkt (gelb), Filterlänge $T=5$ (grüne und rote Punkte), hier liegt eine Zunahme der Herzfrequenz um den betrachteten Wert vor (Abb. aus Lobmaier et al 2012).

Zu jedem Ankerpunkt wird nun ein Zeitfenster von Herzfrequenzwerten der Länge $L$ unmittelbar vor und unmittelbar nach inm festgelegt. Ein Tachogramm hat eine Länge von 2L. Ankerpunkte am Beginn oder am Ende der Aufzeichnung für die es nicht gelingt, ein vollständiges Tachogramm festzulegen, werden aus den Berechnungen ausgeschlossen. Die Tachogramme können, bedingt durch die Nähe der einzelnen Ankerpunkte, überlappen.

Für die Auswahl der Fensterlänge $L$ gilt: $L$ sollte länger als die erwartete Kohärenzzeit der periodischen Signalabschnitte sein und lang genug um die langsamste Schwingung, die detektiert werden soll, miterfassen zu können (vgl. Bauer 2006a). Innerhalb eines Tachogramms $v$ um einen Ankerpunkt ( $i_{v}$ mit $v=$ $0,1 \ldots M$ herum, liegen demnach die in Abbildung 7 aufgeführten Punkte $X$ :

$$
x_{i_{v}-L}, x_{i_{v}-L+1}, \ldots, x_{i_{v}}, \ldots, x_{i_{v}+L-2}, x_{i_{v}+L-1} \text {. }
$$

Abb.7 Punkte innerhalb eines Tachogramms (Abb. aus Bauer 2006a).

Die entstandenen Tachogramme werden nun an ihren Ankerpunkten übereinander gelegt wie in Abb.8A dargestellt. Die Tachogramme werden so in Phase gebracht (vgl. Lobmaier SM 2012). Anschließend werden die Tachogramme gemittelt, dadurch heben sich nicht-periodische Signalanteile wie Artefakte oder Rauschen, die zu den Ankerpunkten nicht phasensynchronisiert sind, auf (vgl. Bauer 2006a). Das gemittelte Tachogramm ist in Abb.8A durch die hervorgehobene schwarze Linie sowie in Abb.8B vergrößert dargestellt. 


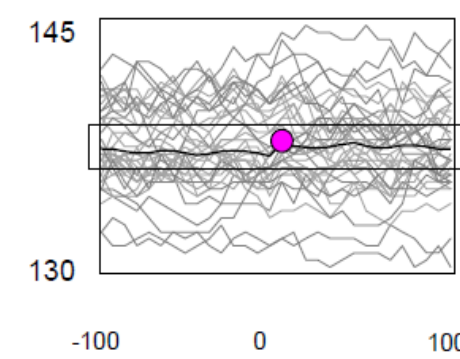

A

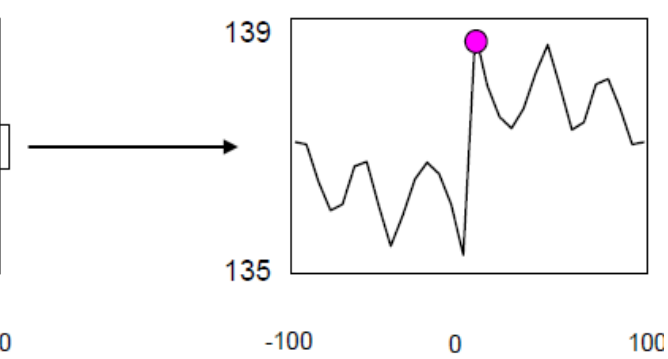

B

Abb. 8A und 8B: Ausrichtung der Tachogramme anhand der Ankerpunkte (A) und Mittelung der Signale (B) (Abb. aus Lobmaier et al 2012).

Die zentrale Amplitude $\mathrm{C}(\mathrm{S})$ ist ein Maß für die dynamische Kapazität des beobachteten Systems (vgl. Lobmaier et al 2012). Stellt ein Anstieg der Herzfrequenz die Bedingung für einen Ankerpunkt dar, so bildet die $\mathrm{C}(\mathrm{S})$ die mittlere Akzelerationskapazität des Systems ab (average acceleration capacity, AAC). Sie berechnet sich, indem man die Mittelwerte von $X$ Werten nach (wobei $\mathrm{i}=0, \ldots \mathrm{X}-1$ ) und $\mathrm{X}$ Werten vor (wobei $\mathrm{i}=-\mathrm{X}, \ldots-1$ ) dem Ankerpunkt voneinander subtrahiert.

Der Parameter AAC kann mittels PRSA sowohl anhand der CTG-, als auch anhand der EKG- Aufzeichnungen bestimmt werden. Die Parameter der PRSA Analyse wurden in dieser Studie entsprechend denen in der Arbeit von Lobmaier SM 2012 ausgewählt (mit einer Fensterlänge L von 100s und einer Zeitintervall $X$ von 10 s) und für die Bestimmung der $A A C$ im CTG, sowie auch im EKG verwendet. Somit berechnet sich die AAC in diesem Fall wie in Abbildung 9 dargestellt:

$$
\sum_{i=0}^{<10 s} \overline{y(i)}-\sum_{\geq-10 s}^{<0} \overline{y(i)}=A A C
$$

Abb.9 Berechnung der AAC (Abb. aus Lobmaier SM 2012).

In der Analyse der fetalen Herzfrequenz mittels PRSA bei wachstumsrestringierten Feten, konnte für die AAC gezeigt werden, dass dieser Parameter eine 
bessere Unterscheidung zwischen wachstumsrestringierten und gesunden $\mathrm{Fe}$ ten ermöglicht als die STV (vgl. Lobmaier et al 2012).

\subsection{Auswertungsmethoden}

\subsubsection{Auswertung der Rohdaten}

Zur Auswertung der Daten für die Dawes Redman Analyse wurde für das CTG die Software Sonicaid Fetal Care (der Firma HNE Huntleigh Nesbit Evans Healthcare GmbH, 35 Portman Moor Road, Cardiff, UK) verwendet, für das fetale EKG die Software Monica DK 1.5b (der Firma Monica Healthcare Ltd. Nottingham, UK).

Zur Bearbeitung der CTG Rohdaten mittels PRSA, wurde das Programm LibRASCH verwendet. Ein Programm, das zur Analyse und Verarbeitung von biologischen Signalen entwickelt wurde (vgl. Schneider et al 2004, vgl. www.librasch.org).

\subsubsection{Statistische Auswertung}

Zur statistischen Datenauswertung wurde die Software Medcalc (Version: MedCalc® v12.7.5, 64-bit, (1993-2013 MedCalc Software bvba) für Windows 7 sowie das Programm SPSS( Version 19 und 21, SPSS Inc., Chicago, IL, USA) verwendet.

Angaben über nominalskalierte Variablen erfolgen in Prozent bzw. für kontinuierliche Größen mittels Median und zugehöriger 25-75\% Perzentile.

Für Berechnungen der Korrelationen wurde Rangkorrelationskoeffizient Rho nach Spearman verwendet. Zum Vergleich gepaarter Stichproben wurde der TTest verwendet. Für die Korrelationen sowie den T-Test sind stets die Anzahl an jeweils gültigen Datenpaaren, die sog. „Sample Size“ (n), die Signifikanz (p), sowie das 95\% Konfidenzintervall angegeben.

Für alle Analysen wurde ein Signifikanzniveau von $p<0,05$ festgelegt. 


\section{Ergebnisse}

\subsection{Patienten}

Es wurden 28 Patientinnen in die Studie eingeschlossen. Insgesamt wurden 112 Messungen durchgeführt. Zwei Patientinnen wurden bereits vor der letzten Messung nach 96 Stunden entbunden.

In Tabelle 1 sind die Teilnehmercharakteristika dargestellt. Das Alter der Patientinnen lag im Median bei 33 Jahren (29,0-35,8). Die Schwangerschaft dauerte ab dem Zeitpunkt der ersten Messung durchschnittlich noch 28,5 Tage (7,3$65,2)$. Hypertensive Schwangerschaftserkrankungen lagen bei $25 \%$ der Teilnehmerinnen vor, 32,1\% erhielten Tokolyse während ihres Krankenhausaufenthaltes. Die häufigsten Gründe für die Betamethasongabe sind in Tabelle 2 dargestellt. Häufigster Grund waren vorzeitige Wehen, Zervixinsuffizienz und IUGR - Situation des Kindes (s. Tabelle 2).

64,3\% der Kinder waren Frühgeburten. Der durchschnittliche Geburts-pH lag bei 7,28 (7,25-7,31) und das Gewicht bei 2117g (1401-3005) (s. Tabelle 1).

\begin{tabular}{l|l} 
Mutter & $\mathrm{n}=28$ \\
\hline Alter (Jahre) & $33,0(29,0-35,8)$ \\
SSW am Tag der ersten Aufzeichnung & $29,6(26,9-31,8)$ \\
SSW bei Entbindung & $34,4(32,0-38,1)$ \\
Schwangerschaftsdauer ab der ersten Messung & $28,5(7,3-65,2)$ \\
in Tagen & $16: 37(13: 41-18: 00)$ \\
Tageszeit der Messungen (MEZ) & $21,3(19,8-24,8)$ \\
BMl vor Schwangerschaft $\left(\mathrm{kg} / \mathrm{m}^{2}\right)$ & $9(32,1 \%)$ \\
Tokolyse &
\end{tabular}




\begin{tabular}{l|l} 
Andere Medikation* & $20(71,4 \%)$ \\
Hypertensive Schwangerschaftserkrankungen: & $7(25 \%)$ \\
Insgesamt & $4(14,3 \%)$ \\
Rauchen & $27(96,4 \%)$ \\
Multipara & $\mathrm{n}=28$ \\
\hline Perinatales Outcome Kind & $18(64,3 \%)$ \\
\hline Frühgeburt & $7,28(7,25-7,31)$ \\
Geburts-pH & $2117(1401-3005)$ \\
Geburtsgewicht (in g) & $17,1(0,3-48,5)$ \\
Geburtsgewichtperzentile & 0 \\
Apgar 5 Min $<7$ & $15(53,6 \%)$ \\
$\begin{array}{l}\text { Aufnahme auf die Neugeborenen Intensiv- } \\
\text { station }\end{array}$ &
\end{tabular}

Tabelle 1: Klinische Charakteristika der Studienteilnehmerinnen.

SSW = Schwangerschaftswoche, PE=Präeklampsie, HELLP $=$ haemolysis, elevated liver enzyme levels, low platelet count. Die Werte sind als Anzahl mit entsprechender Prozentangabe in Klammern bzw. dem Median mit in Klammern stehendem Interquartil Range (25-75\% Perzentile) angegeben. *Die Angabe ", andere Medikation“ beinhaltet: ASS, Heparin, Nifedipin, Magnesium oral oder iv, Fenoterol, sowie Atosiban.

\begin{tabular}{l|l} 
Indikation für Betamethasongabe & $\mathrm{n}=28$ \\
\hline Zervixinsuffizienz & $5(17,9 \%)$ \\
Vorzeitige Wehen & $5(17,9 \%)$ \\
Vorzeitiger Blasensprung & $2(7,1 \%)$ \\
Hypertensive Schwangerschaftserkrankungen & $1(3,6 \%)$ \\
IUGR/SGA & $5(17,9 \%)$
\end{tabular}


Tabelle 2: Indikation zur Betamethasongabe.

IUGR=Inrauterine growth restriction, $S G A=$ small for gestational age. Die Angaben sind absolute Patientenzahlen mit in Klammern stehender Angabe des Prozentanteils am Gesamtkollektiv.

\subsection{Statistischer Vergleich der Mittelwerte zu unterschiedlichen Messzeitpunkten mittels T-Test für gepaarte Stichproben}

Im folgenden Text sowie Abbildungen und Tabellen werden die Begriffe "CTG“ und "Oxford" sowie „fetales EKG" und "Monica“ teilweise synonym verwendet. Die Begriffe "Oxford" und "Monica" beziehen sich auf die (ehemaligen) Herstellernamen "Oxford“ (auf Grund des früheren Herstellers des CTG Gerätes und Analysesoftware, heute: Huntleigh $\mathrm{GmbH}$ ) sowie "Monica“ (Monica Healthcare) und werden im klinischen Sprachgebraucht verwendet.

\subsection{1 Übersicht der Mittelwertveränderungen vor und nach Betamethasongabe}

Die Veränderung der Mittelwerte der Messwerte zwischen den unterschiedlichen Messzeitpunkten dient zur Beschreibung der Entwicklung der Parameter unter Betamethasoneinfluss, sowie dem Vergleich mit den in der Literatur beschriebenen Verläufen der STV (vgl. Kapitel 4.2.1 „Einfluss der antenatalen Kortikosteroidgabe zur Lungenreifeinduktion auf die fetale Herzfrequenzvariabilität“). Eine Übersicht über die Differenz zwischen den Mittelwerten aller untersuchten Parameter (aus den Berechnungen mittels T-Tests für gepaarte Stichproben) ist in Tabelle 3 dargestellt. In den folgenden Kapiteln 3.2.2.-3.2.5. werden die Parameter im Einzelnen ausführlich besprochen. 
Mittelwertdifferenz der Zeitpunkte:

\begin{tabular}{ll|lllll} 
Gerät: & Parameter: & Oh und $24 \mathrm{~h}$ & $24 \mathrm{~h}$ und $48 \mathrm{~h}$ & $48 \mathrm{~h}$ und $96 \mathrm{~h}$ & 0h und $48 \mathrm{~h}$ & 0h und $96 \mathrm{~h}$ \\
\hline CTG & STV $(\mathrm{ms})$ & $+0,77$ & $-1,6^{*}$ & $+0,07$ & $-0,97$ & $-0,79^{*}$ \\
& AAC $(\mathrm{spm})$ & $+0,06$ & $-0,2^{*}$ & $+0,28^{*}$ & $-0,16$ & $+0,07$ \\
\multirow{2}{*}{ Monica } & STV(ms) & $+0,89$ & $-1,07$ & $+0,53$ & $-0,25$ & $+0,05$ \\
& AAC $(\mathrm{spm})$ & $+0,12$ & $-0,15$ & $-0,3$ & $-0,01$ & $-0,23$
\end{tabular}

Tabelle 3: Übersicht: Differenzen der Mittelwerte zwischen den Messzeitpunkten (alle Parameter). Mittelwerte sowie Differenzen sind Ergebnisse der T-Tests für gepaarte Stichproben der Messwerte der jeweiligen Zeitpunkte. Diese sind ausführlich in den Kapiteln 3.2.2 bis 3.2.5. dargestellt. Monica=fetales EKG, ms=Millisekunden, spm=Schläge pro Minute. *signifikante Differenz der Werte (mit $p<0,05)$

Für alle Parameter zeigt sich im T-Test für gepaarte Stichproben eine Abnahme der Mittelwerte zwischen 0 Stunden (h) und 48h, am höchsten ist diese für die STV im CTG $((-0,97) \mathrm{ms})$. Diese Tendenz stimmt mit dem in der Literatur (vgl. Kapitel 4.2.1 „Einfluss der antenatalen Kortikosteroidgabe zur Lungenreifeinduktion auf die fetale Herzfrequenzvariabilität") für die STV im CTG beschriebenen Verlauf überein.

Betrachtet man das Zeitfenster zwischen $0 \mathrm{~h}$ und $48 \mathrm{~h}$ einschließlich des Zeitpunktes $24 \mathrm{~h}$, so zeigt sich zwischen 0h und 24h für alle Parameter zunächst ein tendenzieller Anstieg der Werte, gefolgt von einer Abnahme zwischen 24h und 48h, welche im CTG für AAC und STV signifikant ist (vgl. Tabelle 3, sowie Tabelle 4 bis 7 ).

Zwischen 48h und 96h kommt es erstmals zu einer unterschiedlichen Entwicklung der Werte: ausgenommen der AAC im fetalen EKG, zeigen alle Parameter ein Anstieg der Mittelwerte zwischen diesen Zeitpunkten. Dieser Anstieg ist für die AAC im CTG zwischen 48h und 96h signifikant (Differenz der Mittelwerte $=0,28 \mathrm{spm}$, zweiseitige Signifikanz $\mathrm{p}=0,04)$. Die Mittelwerte der AAC im fetalen EKG hingegen zeigen zwischen $48 \mathrm{~h}$ und $96 \mathrm{~h}$ eine weitere Abnahme um ($0,3)$ spm $(p=0,18)$. 
Im Vergleich der Mittelwerte zwischen 0h und 96h lassen sich zwei Verläufe feststellen: Die Mittelwerte der AAC im CTG, sowie der STV im fetalen EKG zeigen eine tendenzielle Zunahme gegenüber ihren Ausgangsmittelwerten (Oh) um +0,07Spm bzw. +0,05ms. Die Mittelwerte der STV im CTG sowie der AAC im fetalen EKG sind beide, trotz des zunächst erfolgten Anstiegs der STV zwischen 48h und 96h im CTG, jedoch weiterhin gegenüber ihren Ausgangsmittelwerten (entspricht dem Zeitpunkt Oh) zum Zeitpunkt 96h vermindert. Für die STV im CTG ist dieser Unterschied signifikant (Differenz der Mittelwerte= $(-0.79 \mathrm{~ms})$, zweiseitige Signifikanz $\mathrm{p}=0,03)$.

\subsubsection{STV im CTG}

Untersuchte Stichproben= Messwerte der Zeitpunkte:

$$
\text { Oh und } 24 \mathrm{~h} \quad 24 \mathrm{~h} \text { und } 48 \mathrm{~h} \quad 48 \mathrm{~h} \text { und } 96 \mathrm{~h} \quad \text { oh und } 48 \mathrm{~h} \quad \text { Oh und } 96 \mathrm{~h}
$$

\begin{tabular}{l|ccccc}
\hline aM (ms) & 0h:8,86 & $24 \mathrm{~h}: 9,51$ & $48 \mathrm{~h}: 8,17$ & Oh: 8,89 & Oh:9,0 \\
& $24 \mathrm{~h}: 9,63$ & $48 \mathrm{~h}: 7,92$ & $96 \mathrm{~h}: 8,24$ & $48 \mathrm{~h}: 7,92$ & $96 \mathrm{~h}: 8,2$ \\
& & & & & \\
Differenz der & 0,77 & $(-1,59)^{*}$ & $+0,07$ & $(-0,97)$ & $(-0,79)^{*}$ \\
aM (ms) & & & & & \\
Zweiseitige & 0,14 & 0,003 & 0,92 & 0,11 & 0,03 \\
Signifikanz p & & & & & \\
Sample size & 24 & 23 & 20 & 23 & 21 \\
SD & 2,48 & 2,29 & 2,8 & 2,75 & 1,6 \\
$95 \% \mathrm{Cl}$ & {$[(-0.28)-$} & {$[(-2.58)-$} & {$[(-1,25)-$} & {$[(-2,16)-$} & {$[(-1,52)-$} \\
& $1.82]$ & $(-0.60)]$ & $1,38]$ & $0.22]$ & $(-0,06)]$
\end{tabular}

Tabelle 4: Ergebnisse der T-Tests für gepaarte Stichproben: STV im CTG $\mathrm{aM}=$ arithmetisches Mittel, $\mathrm{Cl}=$ conficence interval (Konfidenzintervall), $\mathrm{h}=$ Stunde, ms= Millisekunden, $\mathrm{SD}=$ standard deviation (Standardabweichung).

*signifikante Differenz der Mittelwerte $(m i t p<0,05)$ 
Die Ergebnisse der T-Tests für gepaarte Stichproben für die STV im CTG sind in Tabelle 4 zusammengefasst. Für die Werte von $0 \mathrm{~h}$ und $48 \mathrm{~h}$ zeigte sich eine nicht signifikante, mittlere Differenz der Mittelwerte von $(-0,97)$ ms. Zwischen $24 \mathrm{~h}$ und $48 \mathrm{~h}$ zeigt sich eine signifikante Abnahme der Werte um $(-1,59) \mathrm{ms}$ $(p=0,003)$. Gegenüber dem Mittelwert der Ausgangswerte bei Oh, ist der Mittelwert der STV im Oxford nach 96h im T-Test für gepaarte Stichproben weiterhin signifikant um -0,79ms vermindert. Betrachtet man die Entwicklung der Mittelwerte zwischen $48 \mathrm{~h}$ und $96 \mathrm{~h}$, zeigt sich jedoch auch hier ein tendenzieller Anstieg um 0,07ms (vgl. Tab.4).

\subsubsection{AAC im CTG}

Untersuchte Stichproben= Messwerte der Zeitpunkte:

\section{Oh und $24 \mathrm{~h} \quad 24 \mathrm{~h}$ und $48 \mathrm{~h} \quad 48 \mathrm{~h}$ und $96 \mathrm{~h} \quad$ oh und $48 \mathrm{~h} \quad$ Oh und $96 \mathrm{~h}$}

\begin{tabular}{|c|c|c|c|c|c|}
\hline $\mathrm{aM}(\mathrm{spm})$ & $\begin{array}{l}\text { Oh:2,48 } \\
24 h: 2,53\end{array}$ & $\begin{array}{l}24 h: 2,5 \\
48 h: 2,3\end{array}$ & $\begin{array}{l}48 h: 2,29 \\
96 h: 2,58\end{array}$ & $\begin{array}{l}\text { Oh: } 2,46 \\
48 h: 2,3\end{array}$ & $\begin{array}{l}\text { Oh:2,25 } \\
\text { 96h:2,60 }\end{array}$ \\
\hline $\begin{array}{l}\text { Differenz der } \\
\text { aM (spm) }\end{array}$ & 0,06 & $-0,2^{*}$ & $0,28^{*}$ & $(-0,16)$ & 0,07 \\
\hline $\begin{array}{l}\text { Zweiseitige } \\
\text { Signifikanz p }\end{array}$ & 0,54 & 0,02 & 0,04 & 0,21 & 0,28 \\
\hline Sample size & 23 & 22 & 19 & 22 & 20 \\
\hline SD & 0,44 & 0,37 & 0,56 & 0,57 & 0,30 \\
\hline $95 \% \mathrm{Cl}$ & {$[(-0.13)-0.25]$} & {$[(-0.37)-(-0.04]$} & {$[0,01-0,56]$} & {$[(-0,41)-0,10]$} & {$[(-0,06)-0,21]$} \\
\hline \multicolumn{6}{|c|}{ Tabelle 5: Ergebnisse der T-Tests für gepaarte Stichproben: AAC im CTG } \\
\hline \multicolumn{6}{|c|}{$\begin{array}{l}\mathrm{aM}=\text { arithmetisches Mittel, } \mathrm{Cl}=\text { conficence interval (Konfidenzintervall), } \mathrm{h}=\mathrm{Stunde}, \mathrm{SD}=\text { standard } \\
\text { deviation (Standardabweichung), spm= Schläge pro Minute }\end{array}$} \\
\hline
\end{tabular}

Die Ergebnisse der T-Tests für gepaarte Stichproben für die AAC im CTG sind in Tabelle 5 zusammengefasst. Der Mittelwert der AAC im Oxford ist nach $48 \mathrm{~h}$ 
im Vergleich zum Ausgangswert bei Oh um 0,16spm vermindert. Zwischen 24h und $48 \mathrm{~h}$ zeigt sich ein signifikanter Abfall um 0,2spm. Zum Zeitpunkt 96h unterscheiden sich die Werte im Mittel um 0,07spm vom Ausgangswert bei Oh. Zwischen 48h und 96h zeigt sich ein signifikanter Anstieg der Mittelwerte um $0,28 \mathrm{spm}$ (vgl. Tab.5).

\subsubsection{STV im fetalen EKG}

Untersuchte Stichproben= Messwerte der Zeitpunkte:

Oh und $24 \mathrm{~h} \quad 24 \mathrm{~h}$ und $48 \mathrm{~h} \quad 48 \mathrm{~h}$ und $96 \mathrm{~h} \quad$ Oh und $48 \mathrm{~h} \quad$ Oh und $96 \mathrm{~h}$

\begin{tabular}{l|ccccc}
\hline aM (ms) & 0h:7,77 & $24 \mathrm{~h}: 8,66$ & $48 \mathrm{~h}: 7,55$ & $0 \mathrm{~h}: 7,97$ & 0h:8,12 \\
& $24 \mathrm{~h}: 8,66$ & $48 \mathrm{~h}: 7,58$ & $96 \mathrm{~h}: 8,09$ & $48 \mathrm{~h}: 7,72$ & $96 \mathrm{~h}: 8,17$ \\
& & & & & \\
$\begin{array}{l}\text { Differenz der } \\
\text { aM (ms) }\end{array}$ & 0,89 & $(-1,07)$ & 0,53 & $(-0,25)$ & 0,05 \\
$\begin{array}{l}\text { Zweiseitige } \\
\text { Signifikanz p }\end{array}$ & 0,26 & 0,06 & 0,47 & 0,76 & 0,93 \\
Sample size & 13 & 13 & 12 & 15 & 13 \\
SD & 2,72 & 1,92 & 2,48 & 3,07 & 2,09 \\
95\%Cl & {$[(-0.75)-2.54]$} & {$[(-2.23)-0.08]$} & {$[(-1,04)-2,11]$} & {$[(-1,95)-1,45]$} & {$[(-1,22)-1,31]$}
\end{tabular}

Tabelle 6: Ergebnisse der T-Tests für gepaarte Stichproben: STV im fetalen EKG

$\mathrm{aM}=$ arithmetisches Mittel, $\mathrm{Cl}=$ conficence interval (Konfidenzintervall), $\mathrm{h}=$ Stunde, $\mathrm{ms}=$ Millisekunden, $\mathrm{SD}=$ standard deviation (Standardabweichung).

Die Ergebnisse der T-Tests für gepaarte Stichproben für die STV im fetalen EKG sind in Tabelle 6 zusammengefasst. Die STV im Monica zeigt nach 48h einen Abfall um $(-0,25) \mathrm{ms}$ gegenüber dem Ausgangswert bei Oh, zwischen $0 \mathrm{~h}$ und $24 \mathrm{~h}$ erfolgt zunächst ein Anstieg um $0,89 \mathrm{~ms}$. Zwischen Oh und $96 \mathrm{~h}$ liegt im Mittel ein Anstieg der STV um 0,05ms vor. Zwischen 48h und 96h beträgt die mittlere Differenz der Mittelwerte 0,53ms (vgl. Tab.6). 


\subsubsection{AAC im fetalen EKG}

Untersuchte Stichproben= Messwerte der Zeitpunkte:

\begin{tabular}{|c|c|c|c|c|c|}
\hline & Oh und $24 \mathrm{~h}$ & $24 h$ und $48 h$ & $48 h$ und $96 h$ & Oh und $48 \mathrm{~h}$ & Oh und $96 \mathrm{~h}$ \\
\hline \multirow[t]{2}{*}{ aM (spm) } & Oh:3,91 & $24 h: 4,06$ & $48 h: 3,93$ & Oh: 3,89 & Oh:3,93 \\
\hline & $24 h: 4,03$ & $48 h: 3,91$ & $96 h: 3,63$ & $48 h: 3,88$ & $96 h: 3,69$ \\
\hline $\begin{array}{l}\text { Differenz der } \\
\text { aM (spm) }\end{array}$ & 0,12 & $(-0,15)$ & $(-0,3)$ & $(-0,01)$ & $(-0,23)$ \\
\hline $\begin{array}{l}\text { Zweiseitige } \\
\text { Signifikanz p }\end{array}$ & 0,52 & 0,31 & 0,18 & 0,97 & 0,29 \\
\hline Sample size & 22 & 21 & 19 & 22 & 20 \\
\hline SD & 0,89 & 0,67 & 0,93 & 0,94 & 0,95 \\
\hline $95 \% \mathrm{Cl}$ & {$[(-0.27)-0.52)$} & {$[(-0.46)-0.16]$} & {$[(-0,75)-0,15]$} & {$[(-0,42)-0,41]$} & {$[(-0,68)-0,21]$} \\
\hline
\end{tabular}

Tabelle 7: Ergebnisse der T-Tests für gepaarte Stichproben: AAC im fetalen EKG

$\mathrm{aM}=$ arithmetisches Mittel, $\mathrm{Cl}=$ conficence interval (Konfidenzintervall), $\mathrm{h}=\mathrm{Stunde}, \mathrm{SD}=$ standard deviation (Standardabweichung), spm= Schläge pro Minute

Die Ergebnisse der T-Tests für gepaarte Stichproben für die AAC im fetalen EKG sind in Tabelle 7 zusammengefasst.

Für die AAC im Monica zeigt sich zwischen 0h und 48h eine mittlere Abnahme um $(-0,01)$ spm. Analog zur Entwicklung aller anderen beschriebenen Parameter zeigt sich auch bei der AAC im EKG ein Anstieg zwischen Oh und 24h und eine Abnahme zwischen 24h und 48h. Zwischen 48h und 96h kommt es im Unterschied zum Verlauf der anderen Parameter im Mittel zu einer erneuten Abnahme der AAC um $(-0,3)$ spm. Somit ist die AAC im EKG zum Zeitpunkt 96h im Vergleich zu den Ausgangswerten bei Oh im Mittel noch um 0,23spm vermindert (vgl. Tab.7). 


\subsubsection{Darstellung der Veränderung der STV und AAC im zeitlichen Verlauf mittels Box-Plots}

In Abbildung 10 ist der Verlauf der Messwerte der Kurzzeitvariation (STV) zu den verschiedenen Messzeitpunkten (vor der Betamethasongabe $(=0 \mathrm{~h})$, sowie 24h, 48h und 96h nach der Gabe) für das Oxford CTG (blau, linke Datenreihe) und das fetale EKG (Monica, grün, rechte Datenreihe) abgebildet. Abbildung 11 zeigt den Verlauf der Akzelerationskapazität (AAC) im Oxford (blau, unten) und Monica (grün, oben). Die Mediane der STV sowie AAC zu den verschiedenen Messzeitpunkten sind als Querbalken innerhalb der Boxen dargestellt. Ihr Verlauf verdeutlicht für alle Parameter bis auf die AAC im Monica folgenden Verlauf: zwischen oh und 48h sinkt die STV bzw. AAC, zwischen 48h und 96h erfolgt ein Wiederanstieg.

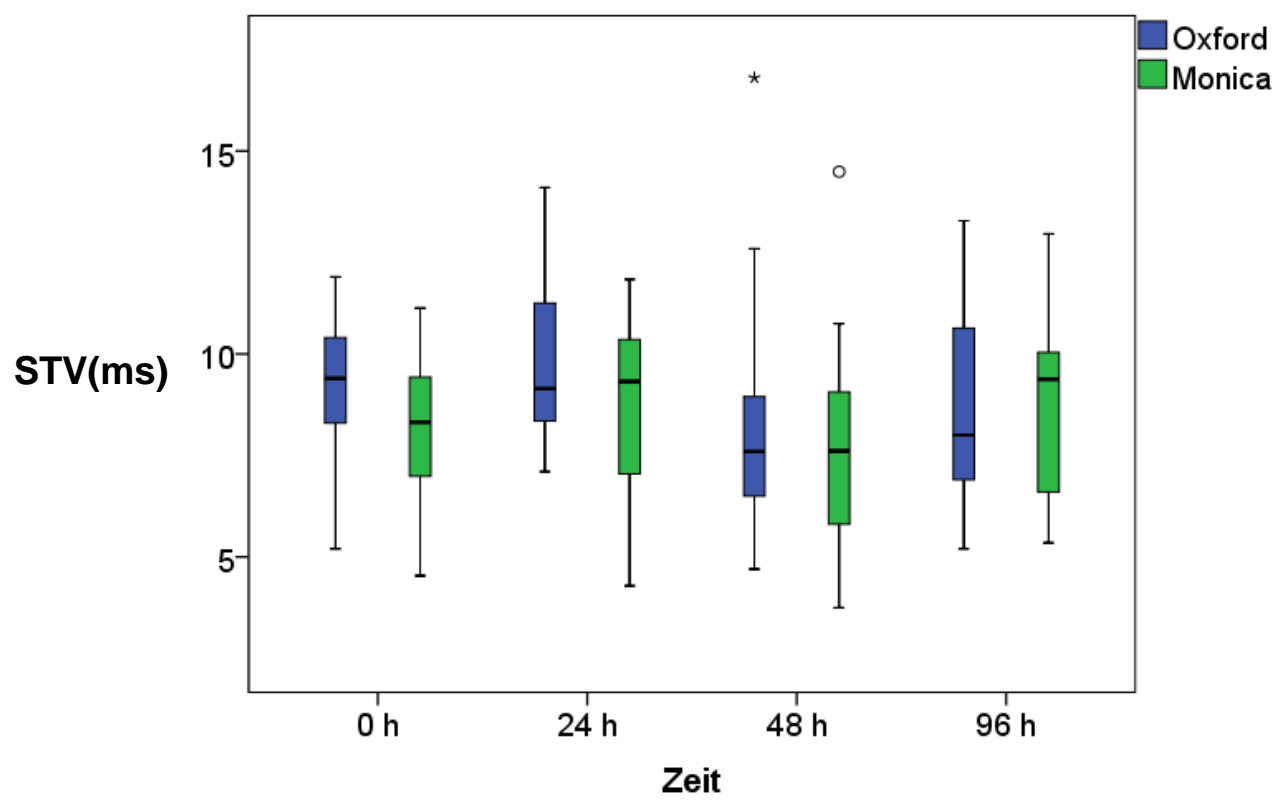

Abb.10: Box- Plots der STV (in ms) im CTG (Oxford) und fetalen EKG (Monica), $n=28$. Für die STV ist im CTG und fetalen EKG eine Abnahme der Werte zwischen oh und 48h sowie ein Wiederanstieg zwischen $48 \mathrm{~h}$ und $96 \mathrm{~h}$ zu erkennen. 


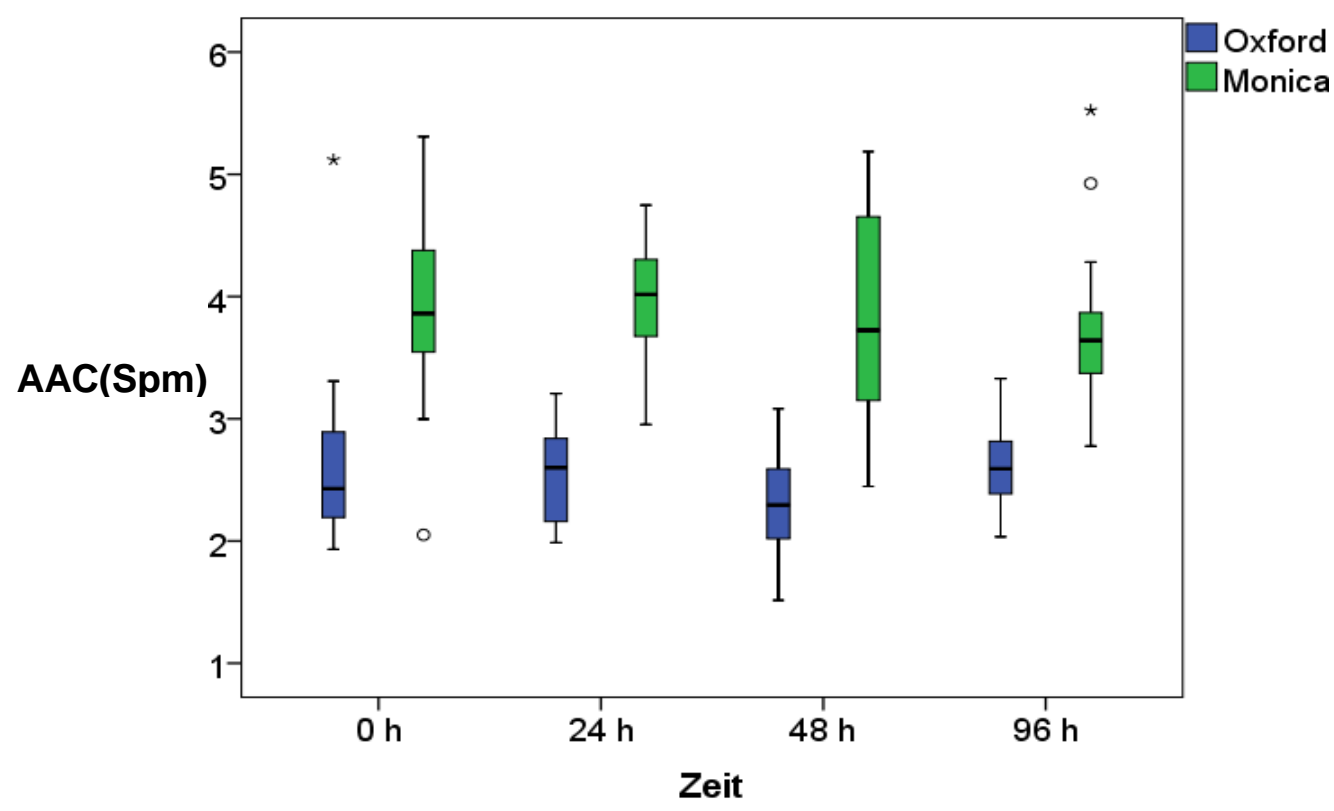

Abb. 11: Box- Plots der Akzelerationskapazität (AAC, Einheit: spm) im CTG (Oxford, untere Reihe) und fetalen EKG (Monica, obere Reihe), $n=28$. Für die AAC im Oxford ist eine Abnahme der Werte zwischen $0 \mathrm{~h}$ und $48 \mathrm{~h}$ sowie ein Wiederanstieg zwischen $48 \mathrm{~h}$ und $96 \mathrm{~h}$ zu erkennen, im T-Test für gepaarte Stichproben zeigte sich eine signifikante Differenz der Mittelwerte der AAC im Oxford zwischen 48h und 96h von $+0,28 s p m \quad(p=0,04)$. Die Abbildung zeigt für das fetale EKG eine Abnahme des Median zwischen 0h und 48h, sowie zwischen 48h und 96h. Im T-Test für gepaarte Stichproben zeigte sich für die AAC im EKG eine Abnahme der Mittelwerte zwischen $0 \mathrm{~h}$ und $48 \mathrm{~h}$, sowie auch zwischen $48 \mathrm{~h}$ und $96 \mathrm{~h}$.

\subsection{Korrelation der Messwerte der Parameter vor und nach Steroidgabe}

\subsection{1 Übersicht der Korrelationen}

In Tabelle 8 sind die Korrelationen der Messwerte aller Parameter zu den drei Messzeitpunkten nach Steroidgabe (24h, 48h, 96h) und ihren Ausgangswerten (Oh) zusammengefasst. In den folgenden Kapiteln 3.3.2 bis 3.3.5. werden diese Korrelationen detailliert dargestellt und erläutert.

Aus Tabelle 8 geht hervor, dass zwischen den Werten vor Steroidgabe (Oh) und den Werten nach 96h bei allen Parametern, bis auf die AAC im fetalen EKG, die Korrelation der Messwerte der jeweiligen Zeitpunkte zu den Ausgangswerten am höchsten ist und dass diese signifikant ist. Im Zeitraum zwischen 24h und 
48h sind die Korrelationskoeffizienten vorübergehend geringer und nicht signifikant.

\begin{tabular}{ll|lll}
\hline Gerät: & Parameter: & Oh und 24h & 0h und 48h & Oh und 96h \\
\hline CTG & STV & 0,21 & 0,28 & $0,69^{*}$ \\
& AAC & 0,25 & $-0,002$ & $0,64^{*}$ \\
EKG & STV & 0,23 & 0,22 & $0,67^{\star}$ \\
& AAC & $-0,04$ & 0,21 & 0,13
\end{tabular}

Tabelle 8: Übersicht der Spearman-Korrelationskoeffizient (Rho) der Werte aller Parameter vor (Zeitpunkt Oh) und nach Betamethasongabe (Zeitpunkte 24h, 48h, 96h). Zum Zeitpunkt 96h zeigt sich bei allen untersuchten Parametern, mit Ausnahme der AAC im fetalen EKG, die höchste Korrelation zu den Ausgangswerten bei Oh. *signifikante Korrelation (mit $p<0,05)$ der Messwerte

Der Einfluss von Betamethason auf die fetale Herzfrequenzvariabilität und ihre Messparameter wird hier in Form der transienten, niedrigen Korrelation der Ausgangswerte zu den Werten der Zeitpunkte 24h und 48h deutlich. Nach 96h wird die fetale Herzfrequenz durch die vorangegangene Steroidgabe nicht mehr relevant beeinträchtigt, wie sich ausgenommen für die AAC im EKG, an der hohen, signifikanten Korrelation der Messwerte zu den Ausgangswerten vor Steroidgabe zeigt.

\subsubsection{STV im CTG}

Die Abnahme des Betamethasoneinflusses auf die STV lässt sich im Oxford CTG durch eine signifikant hohe Korrelation der Werte der Aufzeichnungen vor Kortisongabe (0h) sowie 96h danach abbilden. Zwischen 24h und 48h kommt es zu einer transienten Aufhebung der hohen, signifikanten Korrelation der Messwerte dieses Zeitraums und den Ausgangsmesswerten bei Oh. In den Bland-Altman-Plots in Abb.12- Abb.14 ist dieser Effekt graphisch veranschaulicht.

Tabelle 9 gibt einen Überblick über die Korrelation der Messwerte der STV zu den verschiedenen Zeitpunkten im CTG. 


\begin{tabular}{|c|c|c|c|}
\hline STV im CTG & $\begin{array}{l}\text { Messzeitpunkt } \\
\text { Oh und } 24 \mathrm{~h}\end{array}$ & Oh und $48 \mathrm{~h}$ & Oh und $96 \mathrm{~h}$ \\
\hline Korrelationskoeffizient & 0,21 & 0,28 & $0,69^{*}$ \\
\hline$p$-Wert & 0,34 & 0,19 & 0,0005 \\
\hline Sample size & 24 & 23 & 21 \\
\hline $95 \% \mathrm{Cl}$ & {$[(-0,22)-0,56]$} & {$[(-0,15)-0,62]$} & {$[0,37-0,87]$} \\
\hline
\end{tabular}

Tabelle 9: STV im CTG: Korrelation der Ausgangswerte (Oh) zu den Werten der verschiedenen Messzeitpunkte (24h, 48h und 96h). Die Messwerte von Oh und 96h weisen die höchste Korrelation auf, diese ist signifikant. Zu den Zeitpunkten $24 \mathrm{~h}$ sowie $48 \mathrm{~h}$ ist der Korrelationskoeffizient geringer und nicht signifikant. $\mathrm{Cl}=$ Confidence interval (Konfidenzintervall), Korrelationskoeffizient nach Spearman (Rho), *signifikante Korrelation (mit $\mathrm{p}<0,05)$ der Messwerte

Die Korrelation der Werte zwischen 0h und 24h beträgt 0.21 (vgl. Tab. 9, Sample size $=24$, Spearman-Korrelationskoeffizient $=0.21, p=0.34,95 \% \mathrm{Cl}=[(-0.22)$ 0.56 ), zwischen $0 \mathrm{~h}$ und 48h 0.28 (vgl. Tab. 9, Sample size =23, SpearmanKorrelationskoeffizient $=0.28, p=0.19,95 \% \mathrm{Cl}=[(-0.15)-0.62])$. Sie sind in den Abb.12 und 13 mittels Bland-Altman-Plots dargestellt.

Zwischen den Mittelwerten der STV im CTG zum Zeitpunkt Oh und 96h zeigt sich eine signifikante Korrelation von 0,69 (vgl. Tab. 9, Sample size $=21$, Spearman-Korrelationskoeffizient $=0,69, p=0,0005,95 \% \mathrm{Cl}:[0,37-0,87])$. In Abbildung 14 ist die Korrelation der Werte der STV zu Oh und 96h im Oxford in einem Bland-Altman-Plot dargestellt.

In den Abbildungen ist zu erkennen, dass sich die Punkte in Abbildung 12 und 13 deutlich weiter entfernt um die Mittellinie herum verteilen als in Abbildung 14, welche die Korrelation der Werte von oh und 96 veranschaulicht. Die gemessenen Werte zum Zeitpunkt $0 \mathrm{~h}$ und 24h weichen demnach im Vergleich ihrer Mittelwerte stärker voneinander und somit von ihrer mittleren Differenz (graphisch durch die durchgezogene Mittellinie veranschaulicht) ab, als dies zwischen 0 und $96 \mathrm{~h}$ der Fall ist. Entsprechend ist der Korrelationskoeffizient nach Spe- 
arman (Rho) zum Zeitpunkt Oh und 96h höher als zu den anderen genannten Zeitpunkten.

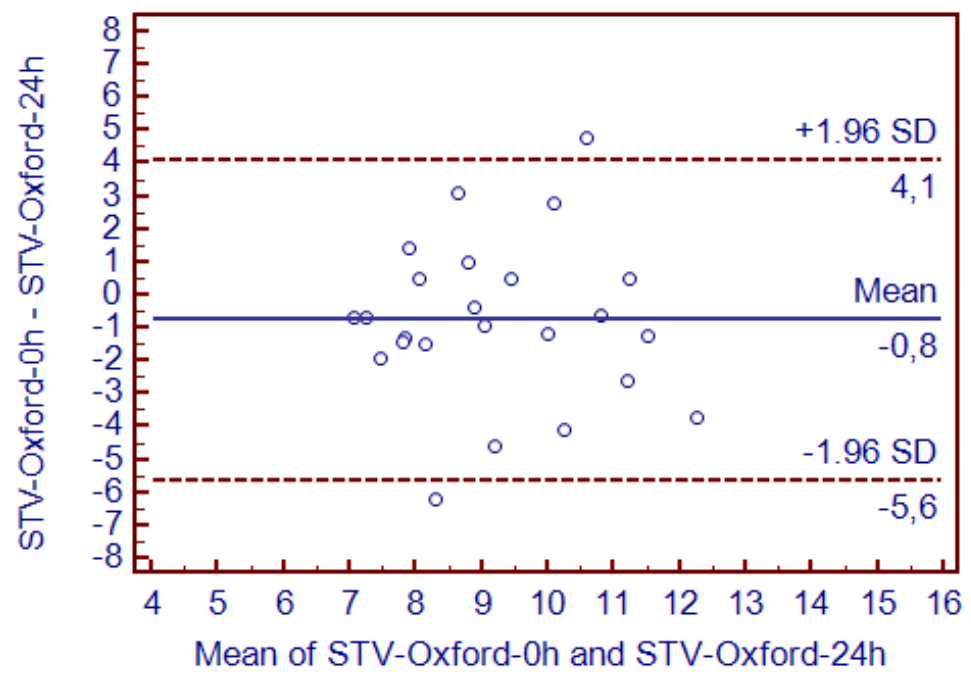

Abb.12: Bland-Altman-Plot: Korrelation der Werte der STV im Oxford zwischen 0h und 24h

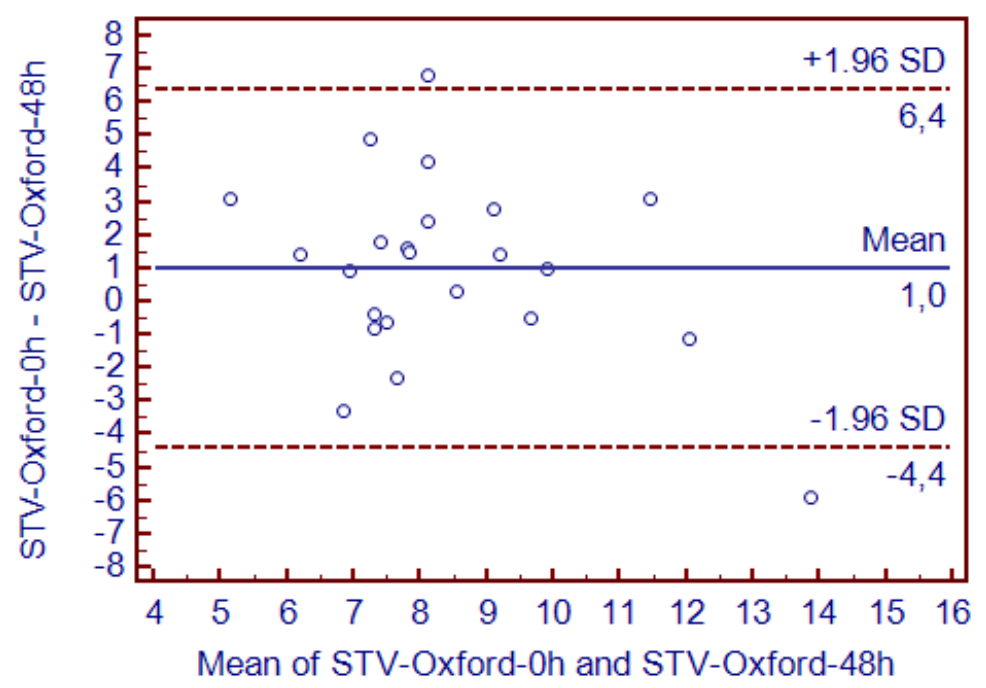

Abb.13 Bland-Altman-Plot: Korrelation der Werte der STV im Oxford zwischen Oh und 48h 


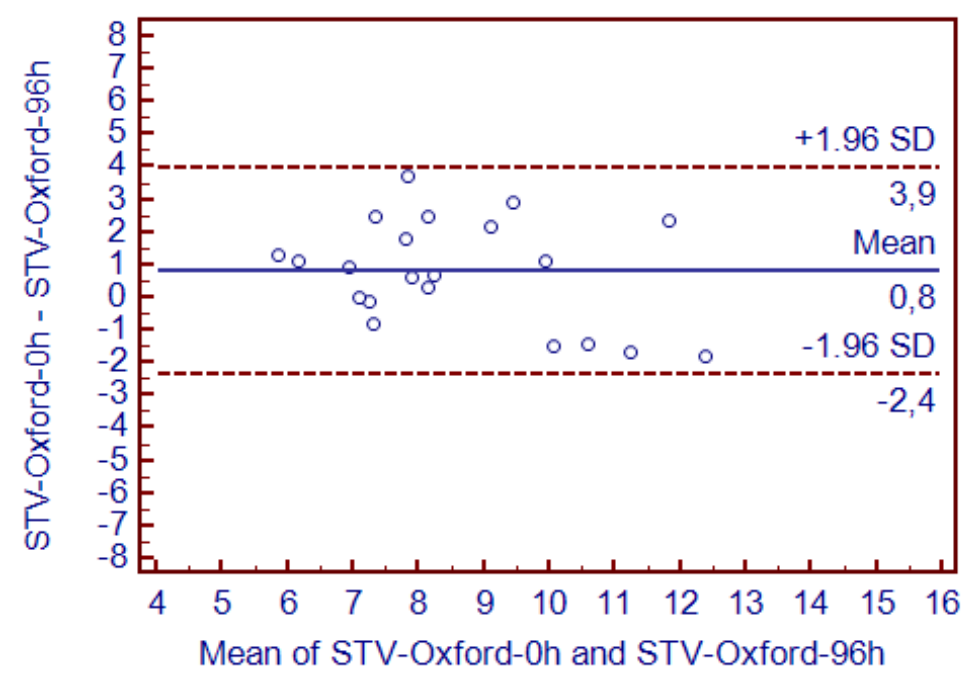

Abb.14 Bland-Altman-Plot: Korrelation der Werte der STV im Oxford zwischen Oh und 96h

\subsubsection{AAC im CTG}

\begin{tabular}{|c|c|c|c|}
\hline AAC im CTG & $\begin{array}{l}\text { Zeitpunkte } \\
\text { Oh und } 24 \mathrm{~h}\end{array}$ & Oh und $48 \mathrm{~h}$ & Oh und $96 \mathrm{~h}$ \\
\hline Korrelationskoeffizient & 0,25 & $-0,002$ & $0,64^{*}$ \\
\hline$p$-Wert & 0,24 & 0,99 & 0,002 \\
\hline Sample size & 23 & 22 & 20 \\
\hline $95 \% \mathrm{Cl}$ & {$[(-0,18)-0,60]$} & {$[(-0,42)-0,42]$} & {$[0,28-0,84]$} \\
\hline
\end{tabular}

Tabelle 10: AAC im CTG: Korrelation der Ausgangswerte (Oh) zu den Werten der verschiedenen Messzeitpunkten (24h, 48h und 96h). Die Messwerte von 0h und 96h weisen die höchste Korrelation auf, diese ist signifikant. Zu den Zeitpunkten $24 \mathrm{~h}$ sowie $48 \mathrm{~h}$ ist der Korrelationskoeffizient geringer und nicht signifikant. $\mathrm{Cl}=$ Confidence interval (Konfidenzintervall), Korrelationskoeffizient nach Spearman (Rho), ${ }^{*}$ signifikante Korrelation (mit $\left.\mathrm{p}<0,05\right)$ der Messwerte

Die Korrelation der AAC im Oxford zwischen den Werten von $0 \mathrm{~h}$ und $24 \mathrm{~h}$ beträgt 0,25 (vgl. Tab. 10, Sample size $=23$, Spearman-Korrelationskoeffizient= $0,25, \mathrm{p}=0,24,95 \% \mathrm{Cl}=[(-0,18)-0,60])$, zwischen $0 \mathrm{~h}$ und $48 \mathrm{~h}$ beträgt sie $(-0,002)$ (vgl. Tab. 10, Sample size $=22$, Spearman- Korrelationskoeffizient $=(-0,002), p=$ $0,99,95 \% \mathrm{Cl}=[(-0,42)-0,42])$. In den Abbildungen 15 und 16 ist die Korrelation der Werte zwischen $0 \mathrm{~h}$ und 24h bzw. Oh und 48h für die AAC im Oxford in Form von Bland-Altmann Plots dargestellt. 
Die Werte der AAC zum Zeitpunkt Oh und 96h korrelieren signifikant im Oxford mit einem Korrelationskoeffizient von 0,64 (vgl. Tab. 10, Sample size =20, Spearman-Korrelationskoeffizient $=0,64, p=0,002,95 \% \mathrm{Cl}=[0,28-0,84])$.

In Abb.17 ist die hohe, signifikante Korrelation der Werte der AAC im CTG zwischen 0h und 96h in einem Bland-Altman Plot dargestellt.

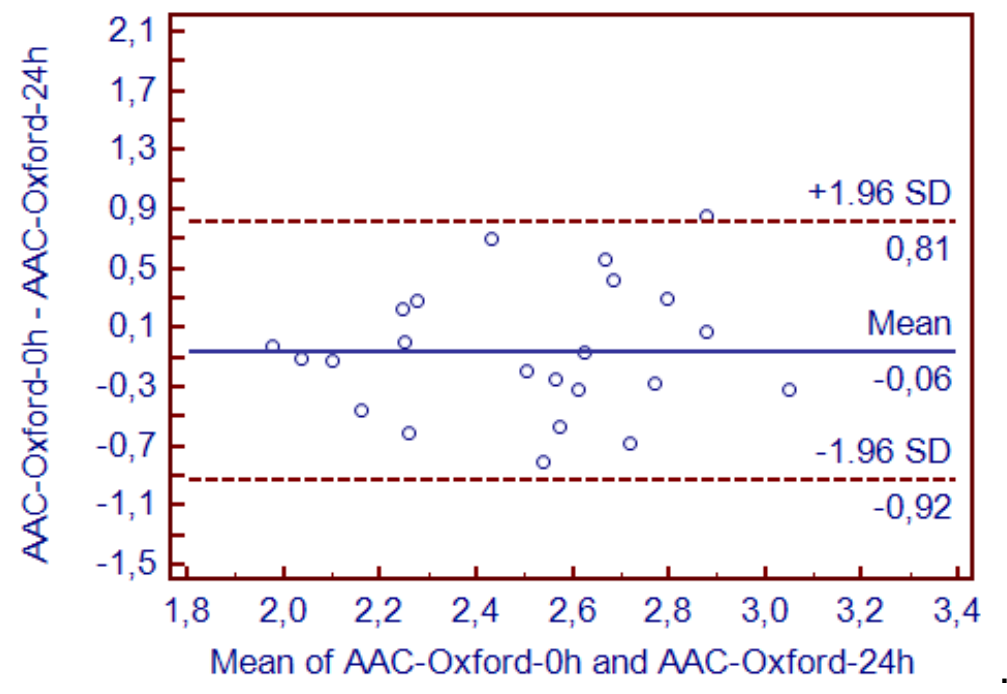

Abb.15 Bland-Altman Plot der Korrelation der Werte der AAC im Oxford zwischen 0h und $24 \mathrm{~h}$.

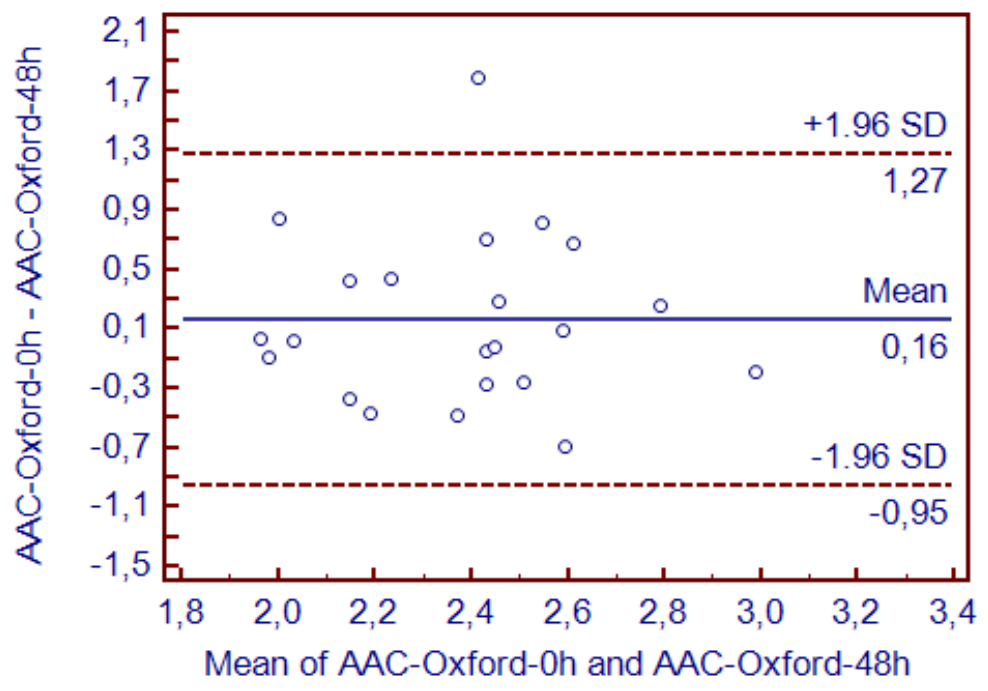

Abb.16 Bland-Altman Plot der Korrelation der Werte der AAC im Oxford zwischen Oh und 48h. 


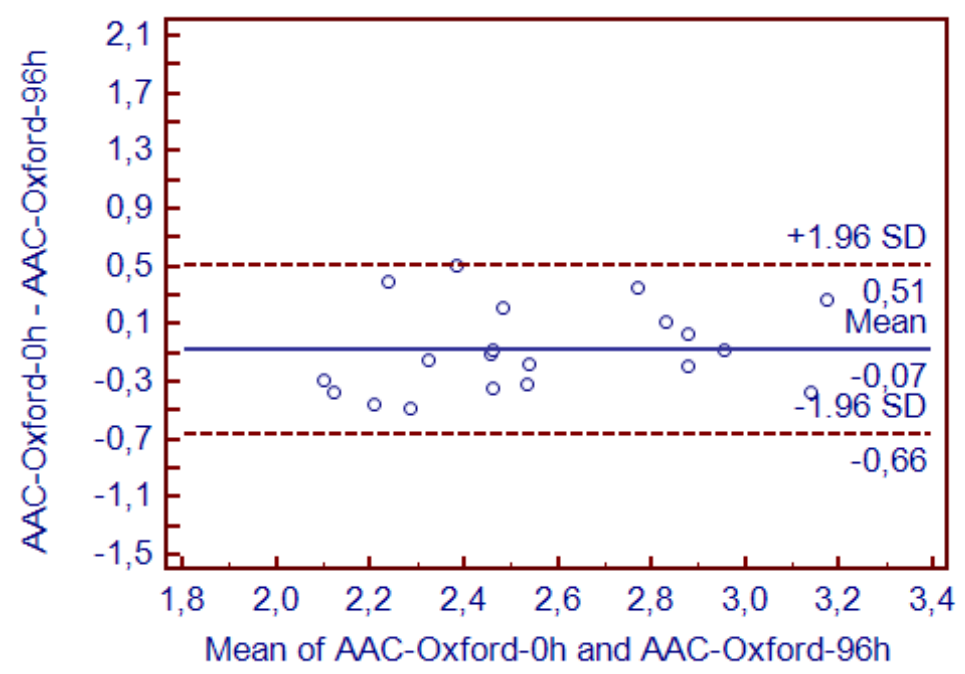

Abb.17 Bland-Altman Plot der Korrelation der Werte der AAC im Oxford zwischen Oh und 96h.

Auch für die AAC im Oxford lässt sich die näher um den Mittelwert gelegene Verteilung der Mittelwertdifferenzen im Vergleich der Zeitpunkte Oh und 96h erkennen, welche der hohen, signifikanten Korrelation der Werte dieser beiden Zeitpunkte entspricht.

\subsubsection{STV im fetalen EKG (Monica)}

\begin{tabular}{l|l|l|l} 
STV im fetalen EKG & \multicolumn{2}{l}{$\begin{array}{l}\text { Messzeitpunkte: } \\
\text { Oh und 24h }\end{array}$} & \multicolumn{2}{|l}{ Oh und 48h } & \multicolumn{2}{l}{ Oh und 96h } \\
\hline Korrelationskoeffizient & 0,23 & 0,22 & $0,67^{*}$ \\
p-Wert & 0,46 & 15 & 0,01 \\
Sample size & 13 & {$[(-0,33)-0,66]$} & 13 \\
$95 \% \mathrm{Cl}$ & {$[(-0,37)-0,69]$} & {$[0,19-0,89]$}
\end{tabular}

Tabelle 11: STV im fetalen EKG: Korrelation der Ausgangswerte (Oh) zu den Werten der verschiedenen Messzeitpunkten (24h, 48h und 96h). Die Messwerte von Oh und 96h weisen die höchste Korrelation auf, diese ist signifikant. Zu den Zeitpunkten $24 \mathrm{~h}$ sowie $48 \mathrm{~h}$ ist der Korrelationskoeffizient geringer und nicht signifikant. $\mathrm{Cl}=$ Confidence interval (Konfidenzintervall), Korrelationskoeffizient Spearman-Korrelationskoeffizient (Rho), *signifikante Korrelation (mit $p<0,05)$ der Messwerte 
Die Korrelation der Werte der STV zu den Zeitpunkten Oh und 24h beträgt 0,23 (vgl. Tab. 11, Sample size $=13$, Spearman Korrelationskoeffizient $=0,23$, $\mathrm{p}=0,46,95 \% \mathrm{Cl}=[(-0,37)-0,69])$, zwischen $0 \mathrm{~h}$ und $48 \mathrm{~h}$ beträgt sie 0,22 (vgl. Tab. 11 , Sample size $=15$, Spearman Korrelationskoeffizient $=0,22, p=0,43$, $95 \% \mathrm{Cl}=[(-0,33)-0,66])$. In Abb. 18 und 19 sind diese Korrelationen in Form von Bland-Altman Plots veranschaulicht. Für das Monica EKG zeigt sich erneut eine signifikante Korrelation von 0,67 zwischen den Werten der STV von Oh und 96h (vgl. Tab. 11, Sample size $=13$, Spearman-Korrelationskoeffizient (Rho) $=0,67$, $\mathrm{p}=0,01,95 \% \mathrm{Cl}=[0,19-0,89])$. In Abb.20 ist die signifikante Korrelation in einem Bland-Altman-Plot dargestellt.

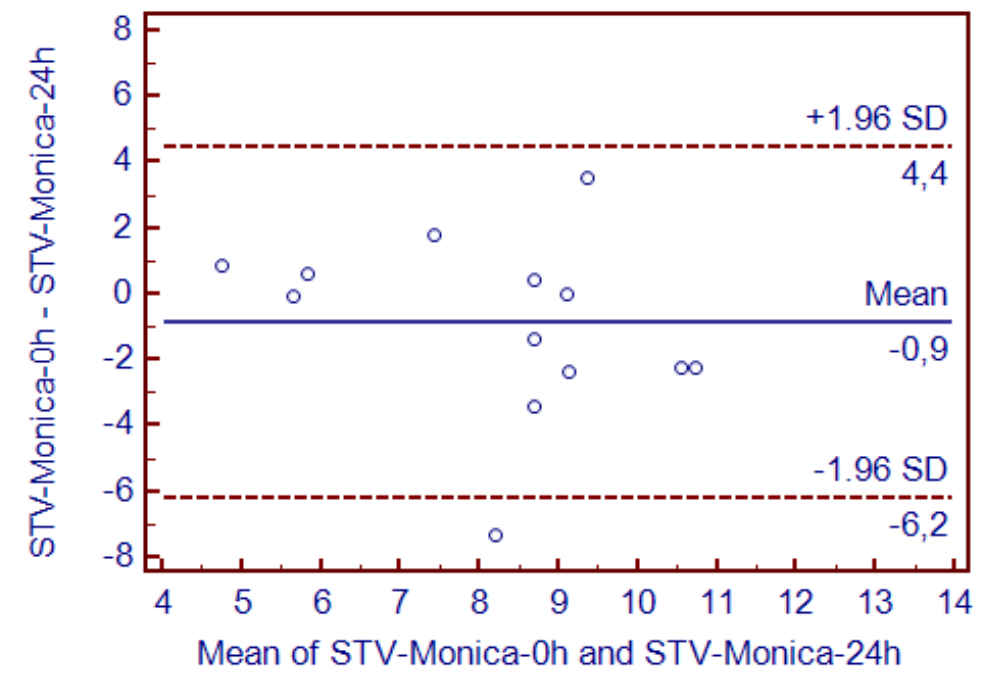

Abb. 18 Bland-Altman-Plot: Korrelation der Werte der STV im fetalen EKG zwischen Oh und 24h

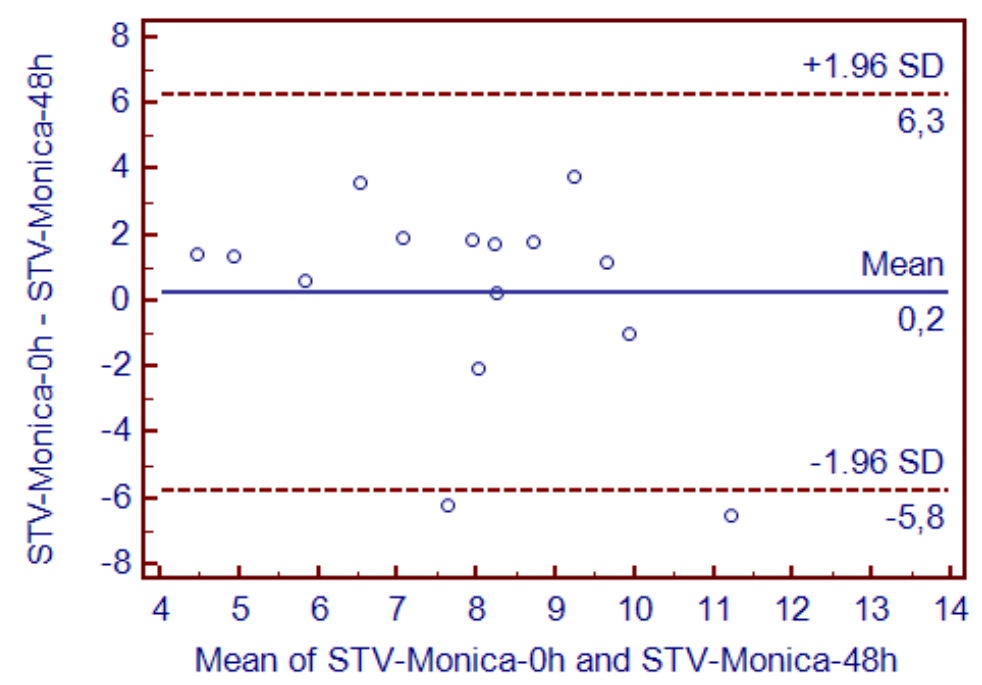

Abb. 19 Bland-Altman Plot: Korrelation der Werte der STV im fetalen EKG zwischen Oh und 48h 


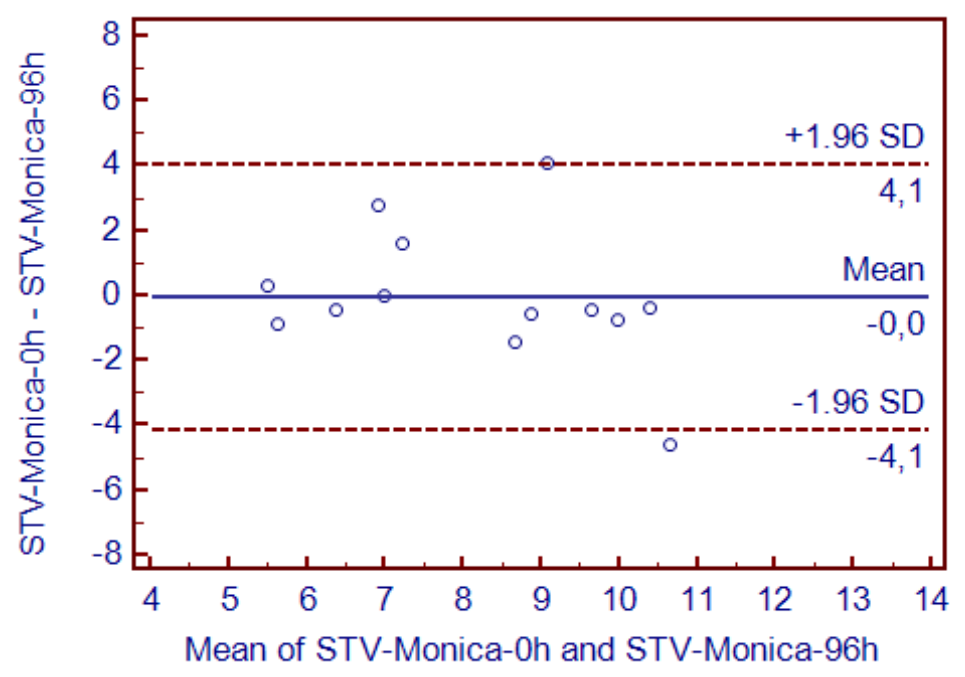

Abb.20 Bland-Altman-Plot: Korrelation der Werte der STV im fetalen EKG zwischen Oh und 96h

\subsubsection{AAC im fetalen EKG (Monica)}

\begin{tabular}{|c|c|c|c|}
\hline AAC im fetalen EKG & $\begin{array}{l}\text { Messzeitpunkte: } \\
\text { Oh und } 24 \mathrm{~h}\end{array}$ & Oh und $48 \mathrm{~h}$ & Oh und $96 \mathrm{~h}$ \\
\hline Korrelationskoeffizient & $-0,04$ & 0,21 & 0,13 \\
\hline $\mathrm{p}$-Wert & 0,86 & 0,35 & 0,56 \\
\hline Sample size & 22 & 22 & 20 \\
\hline $95 \% \mathrm{Cl}$ & {$[(-0,45)-0,39]$} & {$[(-0,23)-0,58]$} & {$[(-0,34)-0,54]$} \\
\hline
\end{tabular}

Tabelle 12: AAC im fetalen EKG: Korrelation der Ausgangswerte (0h) zu den Werten der verschiedenen Messzeitpunkten (24h, 48h und 96h). Die Korrelation der Werte ist zu keinem Zeitpunkt signifikant. $\mathrm{Cl}=$ Confidence interval (Konfidenzintervall), Korrelationskoeffizient nach Spearman (Rho).

Die Korrelation der Werte der AAC im Monica zwischen oh und 24h ist mit -0,04 leicht negativ (vgl. Tab. 12, Sample size $=22$, Spearman Korrelationskoeffizient $=-0,04, p=0,86,95 \% \mathrm{Cl}=[(-0,45)-0,39])$, zwischen 0h und 48h beträgt die Korrelation 0,21 (vgl. Tab. 12, Sample size $=22$, Spearman Korrelationskoeffizient $=0,21, p=0,35,95 \% \mathrm{Cl}=[(-0,23)-0,58])$. Die Korrelationen sind in den Abbildungen 21 und 22 in Form von Bland-Altman Plots dargestellt. 


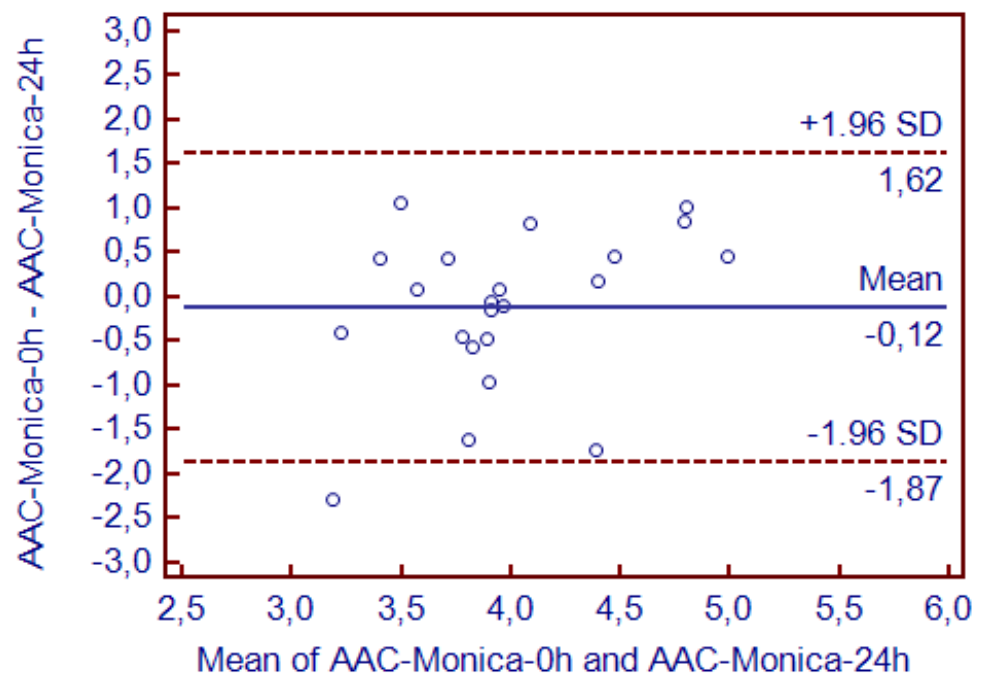

Abb.21: Bland-Altman-Plot: Korrelation der Werte der AAC im Monica zwischen Oh und 24h

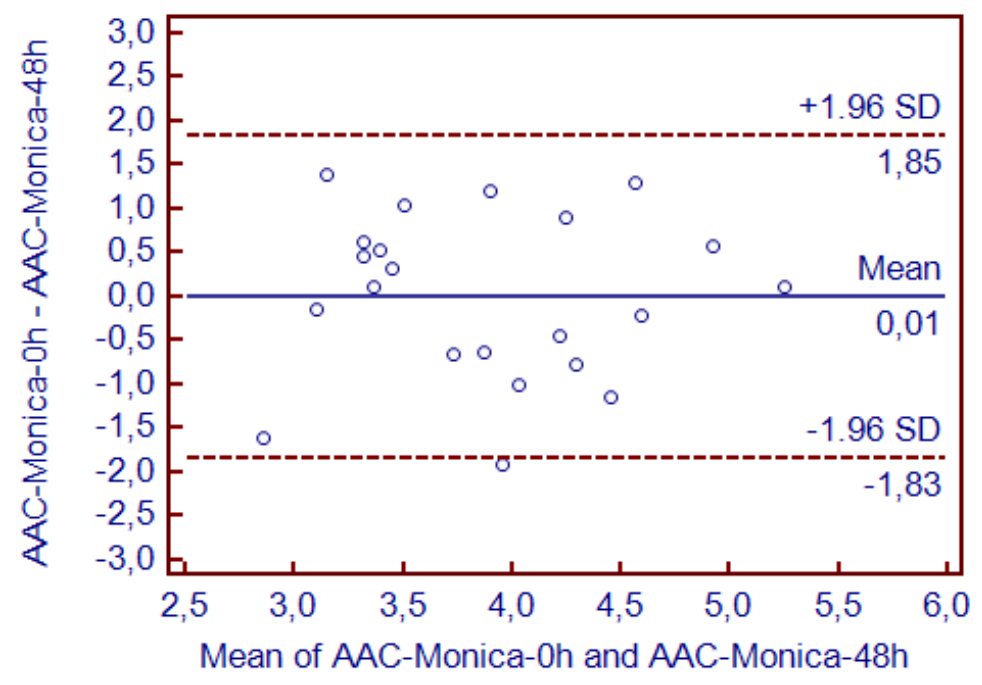

Abb. 22: Bland-Altman-Plot: Korrelation der Werte der AAC im Monica zwischen Oh und 48h

Die Werte der AAC im Monica der Zeitpunkte Oh und 96h zeigen eine Korrelation von 0,13 , in Abb.23 ist diese Korrelation in einem Bland-Altman-Plot dargestellt (Sample size $=20$, Spearman Korrelationskoeffizient $=0,13, p=0,56$, $95 \% \mathrm{Cl}=[(-0,34)-0,54)]$. 


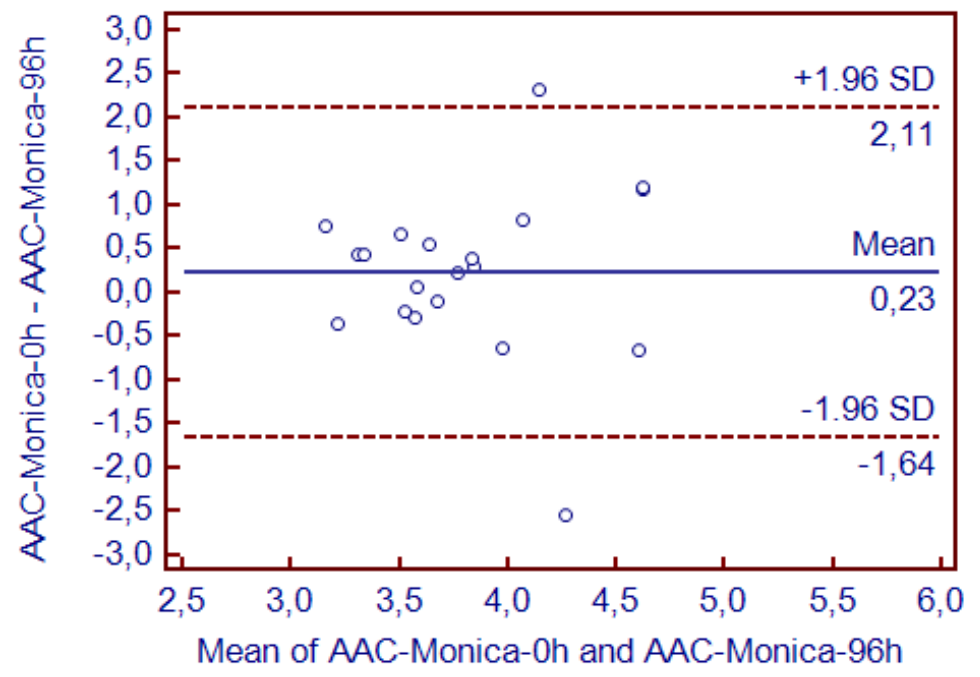

Abb.23 Bland-Altman-Plot: Korrelation der Werte der AAC im Monica zwischen Oh und 96h

\subsection{Korrelation der Parameter STV und AAC zu den vier Messzeitpunkten, Vergleich der Messmethoden}

\subsubsection{Korrelation der Parameter im CTG}

STV und AAC im CTG

\begin{tabular}{|c|c|c|c|c|}
\hline & \multicolumn{4}{|l|}{ Zeitpunkt: } \\
\hline & Oh & $24 h$ & $48 \mathrm{~h}$ & $96 \mathrm{~h}$ \\
\hline Korrelationskoeffizient & $0,42^{*}$ & $0,76^{*}$ & $0,65^{\star}$ & $0,52^{*}$ \\
\hline$p$-Wert & 0,04 & $<0,0001$ & 0,001 & 0,02 \\
\hline Sample size & 25 & 23 & 22 & 20 \\
\hline $95 \% \mathrm{Cl}$ & {$[0,03-0,70]$} & {$[0,50-0,89]$} & {$[0,31-0,84]$} & {$[0,11-0,78]$} \\
\hline
\end{tabular}

Tabelle 13: Korrelation der STV und AAC im CTG.

Spearman-Korrelationskoeffizient (Rho), $\mathrm{Cl}=$ Confidence interval (Konfidenzintervall), *signifikante Korrelation (mit $p<0,05)$ der Messwerte 
Innerhalb des Oxford CTG zeigen die STV und die AAC zu allen vier Messzeitpunkten eine signifikante, positive Korrelation. Eine Übersicht hierüber gibt Tabelle 13. Zum Zeitpunkt Oh beträgt die Korrelation zwischen STV und AAC im Oxford 0,42 (vgl. Tab.13, Sample size $=25$, Spearman-Korrelationskoeffizient (Rho) $=0,42, p=0,04,95 \% \mathrm{Cl}=[0,03-0,70])$, zum Zeitpunkt $24 \mathrm{~h}$ ist sie mit 0,76 am höchsten (vgl. Tab.13, Sample size $=23$, Korrelationskoeffizient $(\mathrm{Rho})=0,76$, $\mathrm{p}<0,0001,95 \% \mathrm{Cl}=[0,50-0,89])$, zum Zeitpunkt $48 \mathrm{~h}$ beträgt sie 0,65 (vgl. Tab.13,Sample size $=22$, Korrelationskoeffizient (Rho) $=0,65, \quad p=0,001$, $95 \% \mathrm{Cl}=[0,31-0,84])$ und bei $96 \mathrm{~h} 0,52$ (vgl. Tab.13,Sample size $=20$, Korrelationskoeffizient $(\mathrm{Rho})=0,52, \mathrm{p}=0,02,95 \% \mathrm{Cl}=[0,11-0,78])$.

\subsubsection{Korrelation der Parameter im fetalen EKG}

STV und AAC

im fetalen EKG

\begin{tabular}{|c|c|c|c|c|}
\hline & \multicolumn{4}{|l|}{ Zeitpunkt: } \\
\hline & Oh & $24 \mathrm{~h}$ & $48 \mathrm{~h}$ & $96 h$ \\
\hline Korrelationskoeffizient & 0,12 & $0,84^{*}$ & $0,6^{*}$ & 0,43 \\
\hline$p$-Wert & 0,65 & 0,0001 & 0,02 & 0,1 \\
\hline Sample size & 16 & 15 & 15 & 17 \\
\hline $95 \% \mathrm{Cl}$ & {$[(-0,4)-0,6]$} & {$[0,57-0,95]$} & {$[0,12-0,85]$} & {$[(-0,07)-0,75]$} \\
\hline
\end{tabular}

Tabelle 14: Korrelation der STV und AAC im fetalen EKG.

Spearman Korrelationskoeffizient (Rho), $\mathrm{Cl}=$ Confidence interval (Konfidenzintervall)

*signifikante Korrelation (mit $\mathrm{p}<0,05)$ der Messwerte

Die Korrelation zwischen den Parametern im fetalen EKG ist in Tabelle 14 dargestellt. Innerhalb des fetalen EKG (Monica) zeigen die Werte der STV und der AAC zum Zeitpunkt 24h und 48h signifikante Korrelationen von 0,84 ( $\mathrm{p}=0,0001$ ) bzw. 0,6 ( $p=0,02)$. Zum Zeitpunkt $0 \mathrm{~h}$ und $96 \mathrm{~h}$ zeigt sich eine positive, allerdings keine signifikante Korrelation. Die Fallzahl ist mit 17 im fetalen EKG zum Zeitpunkt 96h jedoch auch geringer als im CTG mit einer Fallzahl von 20 zum glei- 
chen Zeitpunkt. ( vgl. Tab. 14, Oh: Sample size $=16$, Korrelationskoeffizient (Rho) $=0,12, p=0,65,95 \% \mathrm{Cl}=[(-0,4)-0,6], 24 \mathrm{~h}$ : Sample size $=15$, Korrelationskoeffizient $(\mathrm{Rho})=0,84, \mathrm{p}=0,0001,95 \% \mathrm{Cl}=[0,57-0,95]$, 48h: Sample size $=15$, Korrelationskoeffizient $(\mathrm{Rho})=0,6, \mathrm{p}=0,02,95 \% \mathrm{Cl}=[0,12-0,85]$, 96h: Sample size $=17$, Korrelationskoeffizient $(\mathrm{Rho})=0,43, \mathrm{p}=0,1,95 \% \mathrm{Cl}=[(-0,07)-0,75])$.

\subsection{Korrelation der Parameter zwischen den beiden unterschiedlichen Geräten}

\subsubsection{STV in CTG und fetalem EKG}

STV in CTG und fetalem EKG

\begin{tabular}{l|llll} 
& \multicolumn{4}{l}{ Zeitpunkt: } \\
& $0 \mathrm{~h}$ & $24 \mathrm{~h}$ & $48 \mathrm{~h}$ & $96 \mathrm{~h}$ \\
\hline Korrelationskoeffizient & 0,23 & $0,80^{*}$ & $0,71^{*}$ & 0,2 \\
p-Wert & 0,38 & 0,0002 & 0,002 & 0,45 \\
Sample size & 17 & 16 & 16 & 17 \\
$95 \% \mathrm{Cl}$ & {$[(-0,28)-0,64]$} & {$[0,51-0,93]$} & {$[0,33-0,9]$} & {$[(-0,31)-0,62]$}
\end{tabular}

Tabelle 15: Korrelation der STV in CTG und Monica.

Spearman Korrelationskoeffizient (Rho), $\mathrm{Cl}=$ Confidence interval (Konfidenzintervall)

*signifikante Korrelation (mit $p<0,05$ ) der Messwerte

In Tabelle 15 ist eine Übersicht über die Korrelationen der STV der jeweiligen Zeitpunkte, zwischen den beiden unterschiedlichen Geräten dargestellt. Zu den Zeitpunkten 24h (Korrelationskoeffizient $(R h o)=0,80, p=0,0002)$ und 48 Stunden (Korrelationskoeffizient $(R h o)=0,71, p=0,002$ ) zeigt sich eine hohe, signifikante Korrelation der Werte der STV zwischen den beiden Geräten.

Die Korrelation zum Zeitpunkt Oh (Korrelationskoeffizient $(R h o)=0,23, p=0,38$ ), sowie zum Zeitpunkt 96h (Korrelationskoeffizient $(R h o)=0,2, p=0,45)$ ist hingegen nicht signifikant (vgl. Tab. 15). 


\subsubsection{AAC in CTG und fetalem EKG}

AAC in CTG und

fetalem EKG

\begin{tabular}{l|llll} 
& \multicolumn{4}{|l}{ Zeitpunkt } \\
& Oh & $24 \mathrm{~h}$ & $48 \mathrm{~h}$ & $96 \mathrm{~h}$ \\
\hline Korrelationskoeffizient & 0,02 & $0,43^{*}$ & 0,06 & 0,21 \\
p-Wert & 0,93 & 0,05 & 0,8 & 0,4 \\
Sample size & 24 & 21 & 20 & 19 \\
$95 \% \mathrm{Cl}$ & {$[(-0,4)-0,42]$} & {$[0,001-0,73]$} & {$[(-0,4-0,5]$} & {$[(-0,3)-0,6]$}
\end{tabular}

Tabelle 16: Korrelation der AAC in CTG und Monica.

Spearman Korrelationskoeffizient (Rho), $\mathrm{Cl}=$ Confidence interval (Konfidenzintervall), *signifikante Korrelation (mit $p<=0,05$ ) der Messwerte

Die Korrelation zwischen den Werten der AAC im Oxford und im Monica zu den verschiedenen Messzeitpunkten ist in Tabelle 16 dargestellt. Zum Zeitpunkt 24h ist sie signifikant mit einem Korrelationskoeffizient von 0,43 (Korrelationskoeffizient $(\mathrm{Rho})=0,43, p=0,05)$. Zu den anderen Messzeitpunkten liegt keine signifikante Korrelation vor (vgl. Tabelle 16).

\subsection{Signalverlust}

Sowohl im Oxford CTG als auch im fetalen EKG kann es während einer Aufzeichnung zum Auftreten eines Signalverlustes zwischen 0\% und 100\% kommen.

Zur Berechnung des durchschnittlichen Signalverlustes in den Geräten wurde der Mittelwert verwendet. Tabelle 17 zeigt eine Übersicht des mittleren Signalverlustes zu den verschiedenen Messzeitpunkten. Unter Berücksichtigung fehlender Angaben durch die Software ist in Tabelle 17 der jeweilige Stichprobenumfang $(=n)$ mitangegeben. 
Im fetalen EKG existierten zu 98 Messungen Angaben über den globalen Signalverlust (sog. "Global loss") zwischen $0 \%$ und 100\%. Der durchschnittliche Globale Signalverlust im Monica lag damit bei 33,32\% (Mittelwert, $n=98$ ). Der Analysen-Signalverlust (sog. „Analysis-loss“) lag durchschnittlich bei 58,7\%. Auch hier existierten 98 Angaben zwischen 0\% und 100\%. Der AnalysenSignalverlust liegt damit deutlich höher als der Globale Signalverlust im fetalen EKG.

Im CTG existierten zu 94 Messungen Angaben über den Signalverlust zwischen 0\% und 100\%. Der durchschnittliche Signalverlust liegt hier bei 12\% (Mittelwert, $\mathrm{n}=94)$ und ist damit deutlich geringer als im fetalen EKG.

Wählt man zum Vergleich des Signalverlustes zwischen den Geräten ausschließlich Messzeitpunkte, für die in beiden Geräte Angaben zum Signalverlust zwischen $0 \%$ und $100 \%$ existieren, ergeben sich 91 vergleichbare Messungen. Die hieraus resultierenden Mittelwerte zeigen ebenfalls den deutlich höheren Signalverlust im fetalen EKG. Der durchschnittliche globale Signalverlust liegt hier bei $31,14 \%$, im CTG bei $12,22 \%$.

Im Vergleich zwischen den Geräten zeigt sich insgesamt ein deutlich höherer Signalverlust im fetalen EKG zu allen vier Messzeitpunkten. Der höchste Signalverlust liegt im fetalen EKG zum Zeitpunkt 24h vor (39,2\%), im CTG zum Zeitpunkt Oh (16,1\%).

Für das fetale EKG sind zu jeder Messung sowohl Angaben über den globalen Signalverlust während der Messung (sog. „Global loss") als auch über den Signalverlust bezogen auf die Analyse („Analysis loss“) durch die Software angegeben. Messungen mit einem Globalen Signalverlust von 100\% weisen auch einen Analysen-Signalverlust von 100\% auf. In Messungen mit einem Analysen-Signalverlust von 100\% kann jedoch der globale Signalverlust auch geringer sein. Der analysenbezogene Signalverlust im fetalen EKG zeigt sich für die einzelnen Zeitpunkte mit $66,6 \%$ bei $24 \mathrm{~h}$ am stärksten, mit $51,5 \%$ bei $96 \mathrm{~h}$ am geringsten. Insgesamt liegt der Signalverlust bezogen auf die Analyse zu jedem Zeitpunkt noch einmal deutlich höher als der globale Signalverlust im fetalen EKG. 


\begin{tabular}{|c|c|c|c|c|}
\hline Zeitpunkte & Oh & $24 \mathrm{~h}$ & $48 \mathrm{~h}$ & $96 \mathrm{~h}$ \\
\hline \multicolumn{5}{|l|}{ Fetales EKG } \\
\hline $\begin{array}{l}\text { Vorhandene Anga- } \\
\text { ben zum Signalver- } \\
\text { lust }(=n)\end{array}$ & 28 & 25 & 23 & 22 \\
\hline $\begin{array}{l}\text { Durchschnittlicher } \\
\text { globaler Signalver- } \\
\text { lust (\%) }\end{array}$ & 35,5 & 39,2 & 31,0 & 23,2 \\
\hline $\begin{array}{l}\text { "Durchschnittlicher } \\
\text { Analysen- Signalver- } \\
\text { lust (\%)“ }\end{array}$ & 58,8 & 66,6 & 56,9 & 51,5 \\
\hline \multicolumn{5}{|l|}{ Oxford } \\
\hline $\begin{array}{l}\text { Angaben zum Sig- } \\
\text { nalverlust }(=n)\end{array}$ & 26 & 24 & 23 & 21 \\
\hline $\begin{array}{l}\text { Durchschnittlicher } \\
\text { Signalverlust }\end{array}$ & $16,1 \%$ & $15,5 \%$ & $7,4 \%$ & $7,9 \%$ \\
\hline
\end{tabular}

Tabelle 17: Durchschnittlicher Signalverlust zu den verschiedenen Messzeitpunkten. Der durchschnittliche Signalverlust entspricht dem Mittelwert in Prozent. Unter Berücksichtigung fehlender Angaben zum Signalverlust durch die Software weicht die Grundgesamtheit (=n) zur Berechnung des Mittelwertes teilweise von der Gesamtteilnehmerzahl von 28 wie in der Tabelle angegeben ab. Für das fetale EKG sind sowohl die Angaben zum Globalen Signalverlust einer Messung(sog. „Global loss“), sowie zum Signalverlust bezogen auf die Analyse (sog. „Analysis loss“) aufgeführt. 


\section{Diskussion}

\subsection{Technik}

\subsubsection{Signalverlust im fetalen EKG}

Allgemein ist im fetalen EKG ein Signalverlust auf mehreren Ebenen möglich. Hierbei ist zunächst die Ebene der Rohdatenerhebung selbst zu nennen. Das Gerät AN24 ${ }^{\mathrm{TM}}$ gibt dem Untersucher zu Beginn der Aufzeichnung zwar zu erkennen, ob ein gültiges Signal von Mutter und Kind empfangen werden kann, jedoch kommt es trotz dieser vorangehenden Überprüfung in Einzelfällen zu Signalverlusten im weiteren Verlauf der Aufzeichnung (vgl. Philippe et al 2011). Auch in dieser Studie stellte sich in einigen Fällen nach Übertragung der Daten auf den Computer im Anschluss an die Aufzeichnung ein stattgehabter Signalverlust heraus. Mögliche Ursachen für einen während der Aufzeichnung auftretenden Signalverlust in der Technik des fetalen EKG, stellen die elektrische Isolation des Feten durch die Vernix caseosa, maternale Muskelpotentiale, sowie die mütterliche Herzaktion als Störsignale in der Registrierung der elektrischen Herzaktivität des Feten, dar (vgl. Peters et al 2001).

Im Oxford CTG ist es möglich, die Analyse durch die Software parallel zur Aufzeichnung am Monitor zu verfolgen. Bemerkt der Untersucher während der Aufzeichnung einen Signalverlust, so ist es daher im Unterschied zum fetalen EKG möglich, die Lage des Schallkopfes zu korrigieren und somit dem Signalverlust entgegenzuwirken. Insgesamt lag der globale Signalverlust im fetalen EKG durchschnittlich mit 33,32\% mehr als doppelt so hoch wie im Oxford CTG mit 12\% (vgl. Kapitel 3.6 Signalverlust). Um diesem Problem entgegen zu wirken, ist es empfehlenswert, in künftigen Studien mit dem fetalen EKG, die OnlineÜbertragung der fetalen Herzaktivität mittels WLAN, parallel zur Aufzeichnung, zu nutzen. Diese ist mit der verwendeten Software Monica DK nicht möglich. Damit könnte eine höhere Kontrolle für den Untersucher über die Erfassung des fetalen Signals über die gesamte Aufzeichnungsdauer erreicht werden. 
Weiter muss diskutiert werden, ob es zu einer Beeinträchtigung der Speicherung der Rohdaten auf dem Gerät selbst und konsekutivem Verlust der Rohdaten vor Übertragung auf den Computer gekommen sein kann.

Die letzte Ebene umfasst die Software, in der die Rohdaten gespeichert und analysiert werden. Auch auf dieser Ebene ist das Auftreten möglicher Informationsverluste durch bestimme Analyse-Algorithmen, die Teile der Rohdaten nicht verwenden, denkbar.

Für die Auswertung der Angaben zum Signalverlust durch die Software des fetalen EKG, möchte ich im Folgenden eine Unterscheidung der Messwerte in drei Gruppen vornehmen. Gruppe 1 beinhaltet Messungen, zu denen ein Signalverlust zwischen $0 \%$ und $<100 \%$ durch die Gerätesoftware angegeben werden konnte. Die Ursache des Signalverlustes für diese Messwerte ist demnach am wahrscheinlichsten auf der Ebene der Rohdatenerhebung zu suchen.

Gruppe $2 \mathrm{a}$ enthielt Messungen mit einem globalen Signalverlust von 100\%. Dies betraf acht Messungen. Die Ursache des Signalverlustes aus dieser Gruppe kann auf allen genannten Ebenen liegen.

Gruppe $2 b$ enthielt Messungen mit einem Analysen-Signalverlust von 100\%. Diese Gruppe enthielt daher sämtlichen Messwerten der Gruppe 2a, welche einen globalen Signalverlust von 100\% aufwiesen, sowie darüber hinaus noch 24 weitere Messungen. Für diese 24 Messungen müssen, ergänzend zu den für Gruppe 2a erwähnten möglichen Ursachen des Signalverlustes, Gründe auf Ebene der Software, welche die Analyse durchführt, diskutiert werden.

In Gruppe 3 existierten zu den hier zugehörigen Messungen keinerlei Angaben zum Signalverlust durch die Software. Hierunter fielen, wie auch im CTG, zwei Fälle mit nicht existierenden Rohdaten zur 96h- Messung, auf Grund von Entbindung vor diesem Messzeitpunkt. Darüber hinaus enthielt die Gruppe zwölf weitere Messungen, für elf davon konnte keine AAC und keine STV von der entsprechenden Software berechnet werden. Für diese elf Fälle sind alle oben genannten, möglichen Ebenen auf denen es zu Informationsverlusten kommen kann, als ursächlich zu diskutieren. Für einen der Messwerte ohne Angabe zum Signalverlust konnten im Unterschied zu den 11 beschriebenen Fällen beide 
Parameter, sowohl AAC als auch STV, durch die jeweilige Software berechnet werden. Es ist möglich, dass die Erklärung für diesen Fall auf Ebene der Software liegt, welche den Signalverlust aus den Rohdaten berechnet.

Um die AAC im fetalen EKG besser analysieren zu können ist, neben der bereits genannten Möglichkeit zur Reduktion des Signalverlustes durch WLANÜbertragung der Aufzeichnung, auch die Untersuchung des Parameters bei Feten eines anderen Gestationsalters in Betracht zu ziehen. Zwischen der 28. und 32. SSW ist das Problem der elektrisch isolierenden Vernix caseosa am ausgeprägtesten (vgl. Peters et al 2001). Im Median lag die Schwangerschaftswoche am Tag der ersten Messung (0h) in dieser Studie bei 29,6 SSW.

\subsubsection{Signalverlust im CTG}

Für die Auswertung des Signalverlustes im Oxford CTG möchte ich, wie bereits im fetalen EKG, die Messwerte in zwei Gruppen unterteilen. Gruppe 1 enthielt sämtliche Messungen mit Angaben zum Signalverlust zwischen 0\% und $<100 \%$. Gruppe 2 enthielt 18 Messungen ohne Angaben zum Signalverlust. In dieser Gruppe finden sich neben zwei fehlenden Werten auf Grund von Entbindung vor Abschluss der Messungen zum Zeitpunkt 96h, noch 16 weitere Messungen ohne Angaben zum Signalverlust. Zu sämtlichen Messwerten, für die keine Angaben zum Signalverlust im CTG existieren, konnte auch keine STV oder AAC durch die jeweilige Software berechnet werden.

Eine mögliche Erklärung für diesen Zusammenhang und somit für den Signalverlust in Gruppe 2, muss auf zwei Ebenen diskutiert werden. Zum einen könnte ein vollständiger, vom Untersucher nicht bemerkter Signalverlust während der Aufzeichnung stattgefunden haben. Weiter muss die Ursache fehlender Werte auf Ebene der anschließenden Weiterverarbeitung durch die Software diskutiert werden, welche die Rohdaten speichert und analysiert.

\subsubsection{Bedeutung der Signalverluste für die Studie}

Das abweichende Verhalten der Korrelation der Ausgangswerte zu den Werten zum Zeitpunkt 96h für die AAC im fetalen EKG, ist am ehesten durch den hohen Signalverlust bei Aufzeichnungen mit dem fetalen EKG zu erklären. Bei 
einem durchschnittlichen Signalverlust von über $50 \%$ ist das fetale EKG keine geeignete Methode für Herzfrequenzregistrierung in der 30. SSW, welche in dieser Studie im Mittel vorlag. Dies zeigt sich auch in der fehlenden Korrelation der Parameter STV und AAC in den fetalen EKG Aufzeichnungen (vgl. Tabelle 14). Für die CTG Aufzeichnungen war eine signifikante Korrelation der Parameter hingegen zu allen vier Messzeitpunkten gegeben (vgl. Tabelle 13).

\subsubsection{PRSA-Signalverarbeitung}

Die PRSA-Technik ist momentan nur im Offline-Modus, das heißt im Anschluss an die Rohdatenerhebung, möglich. Eine Online-Analyse befindet sich derzeit in Vorbereitung (vgl. Lobmaier SM 2012). Durch die Durchführung der Analyse im Verlauf der Aufzeichnung, wird eine zeitnahe Beurteilung der berechneten Parameter, beispielsweise der AAC, möglich und somit die Integration des Verfahrens in den klinischen Alltag realisierbar.

\subsection{Physiologie der fetalen Herzfrequenzvariabilität}

Mit dem Begriff der Herzfrequenzvariabilität wird die physiologische Veränderbarkeit des Herzschlages in seiner Geschwindigkeit durch verschiedene, modulierende Einflüsse beschrieben. Sie kann die Schwankung zwischen zwei konsekutiven Herzschlägen darstellen und dann mittels Abstand der R-Zacken der QRS-Komplexe (sogenannter RR-Abstand), beschrieben werden, es kann damit jedoch auch die Schwankung zwischen Herzfrequenzen konsekutiver Zeitabschnitte beschrieben werden (vgl. Task Force of the european society of cardiology 1996).

Beim Erwachsenen wird der modulierende Einfluss durch das autonome Nervensystem, mit seinen beiden Anteilen Sympathikus und Parasympathikus, ausgeübt. Unter Ruhebedingungen ist der parasympathische Einfluss vorherrschend. Außerdem beeinflussen auch Atmung, Barorezeptorreflex und zirkadiane Einflüsse die Herzfrequenzvariabilität.

Beim Erwachsenen steht die Aktivität des autonomen Nervensystems in Zusammenhang mit der kardiovaskulären Mortalität. Eine eingeschränkte Herzfre- 
quenzvariabilität, durch reduzierte vagale Aktivität, ist ein wichtiger Prädiktor des Mortalitätsrisikos nach Myokardinfarkt (vgl. Bauer et al 2006b, vgl. Task Force of the European society of cardiology 1996).

Es gibt verschiedene Methoden die Herzfrequenzvariabilität zu berechnen, wobei die zeitbezogenen (time- domain methods) und die frequenzbezogenen Methoden (frequence- domain methods) unterschieden werden. Bei der zeitbezogenen Methode leiten sich die Messgrößen von sogenannten NN-Intervallen (normal-to-normal-intervals) ab, den Intervallen zwischen konsekutiven QRSKomplexen.

Durch frequenzbezogenen Methoden können verschiedene Komponenten in Spektralanalysen von Kurz- und Langzeitaufzeichnungen betrachtet werden: "very low frequency“ (VLF), "low frequency“ (LF) und "high frequency“ (HF). In Langzeitaufzeichnungen beeinträchtigen nichtstationäre Signale in der Messung die Ergebnisse. Die durch die zeit-, sowie die frequenzbezogenen Messmethoden abgeleiteten Parameter weisen in 24-Stunden Messungen eine hohe Korrelation auf.

Sowohl HF als auch LF Komponenten der Herzfrequenz werden durch vegetative Einflüsse moduliert. Zu den hohen Frequenzkomponenten (HF) trägt der Nervus Vagus vorrangig bei. In Bezug auf das Korrelat der niedrigeren Frequenzkomponenten (LF) wird kontrovers diskutiert, ob es sich in diesem Frequenzbereich um ausschließlich sympathische Modulationen handelt oder ob sich hier der Einfluss beider vegetativer Äste zeigt (vgl. Taskforce of the European Society of Cardiology 1996, vgl. David et al 2007).

Für die Erwachsenen Medizin konnte mit der PRSA Methode ein Parameter entwickelt werden, welcher die Herzfrequenzvariabilität spezifisch bezogen auf Phasen der Dezeleration beschreibt: die Dezelerationskapazität (DC). Eine eingeschränkte DC ist ein Prädiktor für die Mortalität nach Myokardinfarkt und den bislang verwendeten Parametern wie der linksventrikulären Ejektionsfraktion, überlegen. Die DC könnte demnach ein Mittel sein, um die vagale Modulation zu beschreiben (vgl. Bauer et al 2006b). 
In der Geburtshilfe liefert die Herzfrequenzvariabilität Informationen, um das intrauterine Wohlbefinden des Feten abschätzen zu können. Die verwendeten Parameter, um die fetale Herzfrequenz mit ihrer Variation in utero zu beschreiben, sind Kurzzeitvariation, Langzeitvariation, basale Herzfrequenz, Episoden hoher und niedriger Variation, Akzelerationen sowie Dezelerationen. Die fetale Herzfrequenz und ihre Variation stehen in einem inversen Zusammenhang (vgl. (Mulder et al 2004, vgl. Nijhuis et at 1998).

Hon und Lee konnten zeigen, dass bei einer Zustandsverschlechterung des Feten bereits Veränderungen in der Variabilität der Herzfrequenz beobachtet werden können, bevor sich die Herzfrequenz selbst ändert (vgl. Hon et al 1963). Eine reduzierte STV kann auf eine fetale Azidose hindeuten (vgl. Street et al 1991).

Bei einer intrauterinen Zustandsverschlechterung des Feten, zeigen sich ein Anstieg der basalen fetalen Herzfrequenz (FHF), eine Abnahme der STV und LTV und eine Zunahme von Dezelerationen (vgl. Nijhuis et al 2000). Dawes et al beschrieben die Abnahme der STV als ein früher auftretendes Warnzeichen für eine intrauterine Verschlechterung des fetalen Wohlbefindens als das Auftreten von Dezelerationen (vgl. Dawes et al 1992b).

Das Auftreten von Akzelerationen kann Ausdruck fetalen Wohlbefindens sein (vgl. Lee et al 1975). Dezelerative Muster können hingegen mit fetaler Hypoxie oder Azidämie assoziiert sein (vgl. Visser et al 1990).

Die fetale Herzfrequenz und ihre Variabilität werden durch das Gestationsalter, die Tageszeit, den Verhaltenszustand und die Bewegungsaktivität zum Zeitpunkt der Registrierung sowie die Sauerstoffversorgung des Feten beeinflusst. Aber auch Konditionen des mütterlichen Organismus, wie beispielsweise Medikamentengabe, Infektionen, Körperhaltung oder -aktivität während der Registrierung, haben Einfluss auf die fetale Herzfrequenz und die Parameter der Herzfrequenzvariabilität. Die Veränderungen durch antenatale Glucocorticoidgabe zur Induktion der Lungenreifung werden im Abschnitt 4.2.1.„Einfluss der antenatalen Kortikosteroidgabe zur Lungenreifeinduktion auf die fetale Herzfrequenzvariabilität" ausführlich beschrieben. Über den Sauerstoffgehalt im mütterlichen Blut sowie Veränderungen der uteroplazentaren Durchblutung kann das 
veränderte Sauerstoffangebot im fetalen Organismus direkt zu Veränderungen der fetalen Herzfrequenz führen. Eine chronische Hypoxie, wie beispielsweise bei einer IUGR-Situation, führt jedoch auch über zentrale Regulationsmechanismen zu einer Variation der fetalen Verhaltenszustände, so verbringen IUGRFeten weniger Zeit im aktiven Wachzustand als unauffällige Feten, um den Sauerstoffverbrauch zu verringern (vgl. Schneider K.T.M, Gnirs J. 2011). Nijhuis et al teilten fetales Verhalten in die Kategorien $1 \mathrm{~F}$ bis $4 \mathrm{~F}$ ein. Innerhalb der Kategorien können unter anderem auch unterschiedliche Muster der fetalen Herzrate beschrieben werden. So zeigen sich vor allem im Stadium $1 \mathrm{~F}$ ähnliche Herzfrequenzvariationen, im Sinne einer eingeengten Oszillationsamplitude, wie sie auch bei fetaler Hypoxie auftreten können. Die Beurteilung des fetalen Wohlbefindens durch den Untersucher wird so, auch auf Basis einer computerisierten CTG-Analyse, erschwert (vgl. Nijhuis et al 1982, vgl. Mantel et al 1991, vgl. Schneider K.T.M., Gnirs J. 2011).

Ein reifer Fetus verbringt durchschnittlich ein Drittel der Zeit im Zustand 1F, von einigen Autoren als Tiefschlafperiode bezeichnet. Für eine Differenzierung zwischen pathologischen Herzfrequenzmustern und einer Tiefschlafperiode ist eine Aufzeichnungsdauer von mindestens 40 Minuten notwendig (vgl. Pillai et al 1990a, vgl. Schneider K.T.M, Gnirs J. 2011). Zu "coherent behavioural states“ (s. Nijhuis et al 1982, S.192), den Verhaltenszustände im engeren Sinne, formieren sich die beteiligten Variablen erst ab einem Gestationsalter zwischen 36. und 38 SSW. In früheren Stadien der Schwangerschaft sollte daher korrekterweise lediglich von einer "Koinzidenz der Stadienparameter" gesprochen werden (s. Nijhuis et al 1982, S.192).

Ab der 24. SSW fällt die mittlere fetale Herzfrequenz mit zunehmendem Gestationsalter ab (vgl. Visser et al 1981, Serra et al 2009).

Der Anteil der Akzelerationen nimmt im Verlauf der Schwangerschaft zu, ab der 30. SSW überwiegen sie gegenüber dem Auftreten von Dezelerationen (vgl. Visser et al 1981). Auch der Anteil an Bewegungen des Feten, die von Akzelerationen begleitet werden, sowie die Amplitude dieser Akzelerationen, nehmen mit steigendem Gestationsalter zu (vgl. Pillai et al 1990b). 
Ab der 25. SSW nehmen die STV, LTV und Episoden hoher sowie niedriger Variation ebenfalls zu. Ab der 42. SSW kehren sich die beschriebenen Tendenzen sowohl für die genannten Parameter als auch die basale fetale Herzfrequenz (FHF) um (vgl. Serra et al 2009). Bei IUGR- Feten bestehen eine höhere basale FHF, sowie eine geringere STV und LTV als beim Normalkollektiv (vgl. Nijhuis et al 2000). Eine Abnahme der Herzfrequenzvariabilität kann zwar auch bei IUGR- Feten auf eine Zustandsverschlechterung hinweisen, jedoch ist dies eher ein spätes Zeichen (vgl. Street et al 1991).

Analog zur DC bei Erwachsenen konnte mittels der PRSA Methode für den Fetus ein neuer Parameter, die Akzelerationskapazität AAC, entwickelt werden, welcher die Herzfrequenzvariabilität bezogen auf Phasen der Akzeleration beschreibt. Lobmaier und Huhn konnte zeigen, dass die Beeinträchtigung der Herzfrequenzvariabiliät bei IUGR- Feten sich mit der AAC sehr genau abbilden lässt und diese Feten mit diesem Parameter zuverlässiger erkannt werden können, als mit dem bislang verwendeten Parameter STV ( $\mathrm{vgl}$. Lobmaier et al 2012, vgl. Huhn et al 2011).

Jensen et al beschrieben 2009 bei Schaf-Feten beide Äste des vegetativen Nervensystems als verantwortlich für die fetale Herzfrequenzvariabilität in utero (vgl. Jensen et al 2009).

Assali et al vermuten, dass neben dem autonomen Nervensystem am Herzen von Lammfeten für Veränderungen auf Schlag zu Schlag Ebene auch die intrinsische Myokardaktivität durch Aktionspotentialänderungen eine Rolle spielt (vgl. Assali et al 1977).

Die Anteile des vegetativen Nervensystems und ihr Einfluss auf die fetale Herzfrequenz entwickeln sich zeitlich verschieden voneinander. Manning beschreibt den Einfluss des vegetativen Nervensystems beim Feten auf die Herzaktivität ab der 16.SSW. Zum initialen, alleinigen Einfluss des Sympathikus tritt ab der 18.SSW der parasympathische Anteil des autonomen Nervensystems frequenzmodulierend hinzu. Etwa zu diesem Zeitpunkt ist zum ersten Mal eine Herzfrequenzvariabilität, zunächst in Form der Kurzzeit- und im Verlauf auch der Langzeitvariation, messbar (vgl. Manning 1995, S.15-18). 
Neben der Frage nach dem zeitlichen Entstehungsverlauf des vegetativen Nervensystems, stellt sich bei Fetus, wie auch beim Erwachsenen, die Frage nach einer möglichen Zuordnung der Messparameter zu den einzelnen, autonomen Anteilen Sympathikus und Parasympathikus. David et al beobachteten verschieden schnelle Anstiege der Frequenzkomponenten HF, LF und VLF im Verlauf der Schwangerschaft. Im Hinblick auf die Erkenntnisse am erwachsenen Herzen und durch Tierexperimente vermuten sie, dass sowohl LF als auch HF Komponenten beim Feten sympathische und parasympathische Anteile haben. Die VLF Komponenten bilden hingegen lediglich die sympathische Innervation ab (vgl. David et al 2007, vgl. Akselrod et al 1981).

\subsubsection{Einfluss der antenatalen Kortikosteroidgabe zur Lungenreifeinduktion auf die fetale Herzfrequenzvariabilität}

In der Dawes- Redman Analyse werden verschiedene Parameter bestimmt, um die fetale Herzratenvariabilität zu analysieren. Dabei ist ein zentraler Parameter die Kurzzeitvariation (STV). Die Auswirkungen der Kortikosteroidgabe auf die STV wurden von mehreren Autoren untersucht.

In den meisten Studien zeigte sich eine Abnahme der STV 48 Stunden nach Steroidgabe. Nach 96 Stunden zeigte sich eine Rückkehr in Richtung der Ausgangswerte, die beobachteten Veränderungen sind somit lediglich transient. Derks et al fanden 48 Stunden, sowie 72 Stunden nach Betamethasongabe eine signifikant reduzierte STV sowie LTV, die nach 96 Stunden jedoch wieder Ausgangswerte erreicht hatten (vgl. Derks et al 1995).

Senat et al wiesen ebenfalls nach Betamethasongabe eine signifikante Abnahme der STV nach 24-48 Stunden nach der Gabe nach. Vier bis sieben Tage später erreichte sie wieder Ausgangwerte (vgl. Senat et al 1998).

Rotmensch et al wiesen sowohl für Dexamethason, als auch für Betamethason eine transiente Reduktion der STV und LTV 48 Stunden nach der Gabe nach, wobei die Abnahme unter Betamethason prozentual stärker war. Nach 96 Stunden wurden auch hier in beiden Gruppen wieder Ausgangswerte beobachtet 
(vgl. Rotmensch 1999a). In ihrer Untersuchung von 2005 konnte für Betamethason alleine bestätigt werden, dass hierunter die fetale Herzfrequenzvariabilität 48-72 Stunden nach der ersten Gabe signifikant reduziert war. Nach 96 Stunden zeigte sich eine Tendenz in Richtung der Ausgangswerte (vgl. Rotmensch et al 2005).

Brownfoot et al kamen in der Analyse der Daten von Rotmensch et al 1999, Senat et al 1998 und Magee et al 1997 zu dem Ergebnis, dass in Gruppen die Dexamethason erhalten hatten, die fetale Herzfrequenz nach 48 Stunden signifikant stärker reduziert war, als in Gruppen, die Betamethason erhalten hatten (vgl. Brownfoot et al 2008).

Lunshof et al fanden innerhalb der ersten zwölf Stunden nach Betamethasongabe einen Anstieg der STV, sowie der LTV. Im Zeitraum zwischen 42 und 48 Stunden zeigte sich auch hier eine Abnahme der beiden Parameter (vgl. Lunshof et al 2005).

Koenen et al konnten ebenfalls im Zeitraum zwischen 48 und 72 Stunden nach der Gabe von Betamethason eine Abnahme der Herzfrequenzvariabilität, sowie nach 96 Stunden einen Anstieg der STV und LTV nachweisen (vgl. Koenen et al 2005).

In der vorliegenden Studie wurden die Mittelwertverläufe der STV im CTG und fetalen EKG mittels T-Test für gepaarte Stichproben mit den in der Literatur beschriebenen Verläufen der STV unter Betamethasoneinfluss im CTG verglichen. Darüber hinaus wurde auch der Verlauf der Mittelwerte der AAC in beiden Geräten verglichen.

Für die STV fand sich in der vorliegenden Studie sowohl im CTG, als auch im fetalen EKG eine Abnahme der Mittelwerte zwischen zwischen 0h und 48h bzw. zwischen 24h und 48h. Im CTG war die Abnahme der STV zwischen 24h und 48h signifikant. Nach 96h zeigte sich im CTG ein tendenzieller Anstieg des Mittelwertes der STV, im fetalen EKG ebenso. Diese Verläufe stimmen mit der in der Literatur für die STV im CTG nach 96h beschriebenen Entwicklung überein. Für die AAC zeigte sich in der vorliegenden Studie im CTG und im fetalen EKG ebenfalls eine tendenzielle Abnahme der Mittelwerte zwischen 0h und 48h. 
Zwischen 24 und 48h war die Abnahme des Mittelwertes der AAC im CTG signifikant. Nach dem Zeitpunkt 48h stimmt lediglich der Verlauf der Mittelwerte der AAC im CTG, mit den in der Literatur beschriebenen Verläufen der STV überein, hier zeigte sich zwischen $48 \mathrm{~h}$ und $96 \mathrm{~h}$ ein signifikanter Anstieg des Mittelwerts. Keine Übereinstimmung fand sich hingegen für den Verlauf der AAC im fetalen EKG. Hier zeigte sich zwischen 48h und 96h weiterhin eine tendenzielle Abnahme des Mittelwertes.

Dieser Mittelwertverlauf unterscheidet sich von dem der anderen Parameter nach 48h. Als mögliche Ursache hierfür ist der oben genannte hohe Signalverlust im fetalen EGK denkbar.

In der vorliegenden Studie erfolgte darüber hinaus die Berechnung der Korrelationen der Ausgangsmesswerte der Parameter zu den Messwerten der drei Folgezeitpunkte. Der Verlauf der Korrelation wurde mit den in der Literatur beschriebenen Veränderungen der Herzratenvariabilität unter Steroideinfluss zu den verschiedenen Messzeitpunkten verglichen.

Die Korrelation der Werte der Zeitpunkte $0 \mathrm{~h}$ und $24 \mathrm{~h}$ bzw. Oh und $48 \mathrm{~h}$ war für die STV im CTG, sowie im fetalen EKG gering und nicht signifikant.

Nach 96 Stunden hingegen lag für die STV, sowohl im CTG als auch im fetalen EKG eine höhere und signifikante Korrelation der Messwerte mit den Ausgangswerten vor Steroidgabe vor.

Im Zeitraum zwischen 24h und 48h wird die STV durch die Betamethasongabe beeinflusst. In diesem Zeitraum ist für die STV im CTG und im fetalen EKG eine transiente, niedrige Korrelation der Ausgangsmesswerte mit den Messwerten dieses Messzeitraumes zu beobachten.

Nach 96h kommt es zu einer Rückkehr der Werte der STV in Richtung des Ausgangsniveaus vor Steroidgabe. In der vorliegenden Studie korrelieren die Messwerte der STV, sowohl im CTG als auch im fetalen EKG, zum Zeitpunkt 96h höher und signifikant mit ihren Ausgangsmesswerten, als dies noch zu den vorangegangenen Messzeitpunkten der Fall ist.

Die Korrelation der Ausgangsmesswerte der AAC im CTG mit Werten zum Zeitpunkt $24 \mathrm{~h}$ ist ebenfalls nur gering, zwischen oh und $48 \mathrm{~h}$ zeigt sich eine 
leicht negative Korrelation. Nach 96h besteht auch für die AAC im CTG eine höhere und signifikante Korrelation der Werte zu den Ausgangswerten vor Steroidgabe.

Sowohl für die STV im CTG und fetalen EKG, als auch für die AAC im CTG lässt sich der Steroideinfluss auf die fetale Herzfrequenzvariabilität durch die Korrelation der Werte, entsprechend der in der Literatur beschriebenen zeitlichen Ausprägung der Veränderungen der STV durch die Steroidgabe, nachvollziehen. Zum Zeitpunkt 96h zeigt sich eine hohe, signifikante Korrelation der Messwerte zu ihren Ausgangswerten vor Steroidgabe, zu diesem Zeitpunkt besteht demnach keine relevante Beeinflussung der Korrelation durch die Steroidgabe mehr. Zwischen 24h und 48h korrelieren die Messwerte lediglich gering mit ihren Ausgangswerten. Dies entspricht zeitlich dem in der Literatur anhand der STV beschriebenen, passageren Einfluss von Betamethason auf die Herzfrequenzvariabilität.

Im fetalen EKG zeigt die Korrelation der Ausgangswerte der AAC zu den gemessenen Werten zum Zeitpunkt 96h einen anderen Verlauf, es erfolgt zu diesem Zeitpunkt keine Rückkehr zu einer höheren, signifikanten Korrelation. Zum Zeitpunkt 96h zeigt sich hingegen die geringste Korrelation zu den Ausgangswerten im Vergleich der Messzeitpunkte.

Für die AAC im fetalen EKG sollte dennoch analog zum CTG angenommen werden, dass sie zum Zeitpunkt 96h nicht mehr relevant durch die Steroidgabe beeinflusst wird. Mögliche Gründe für einen fehlenden Nachweis in der vorliegenden Studie sind zum einen die niedrige Fallzahl, als auch in der hohe Signalverlust in den fetalen EGK- Aufzeichnungen.

Von Senat et al wurde bei der Untersuchung des Einfluss von Betamethason auf die STV die fetale HRV teilweise über den Zeitpunkt von 96h hinausgehend, zwischen 96h und sieben Tagen untersucht (vgl. Senat et al 1998). Eine entsprechende Verlängerung des Messzeitraumes über 96h hinaus zur weiteren Beobachtung der AAC wäre in künftigen Studien ein interessanter Ansatz, um zu untersuchen, ob die niedrige Korrelation zum Zeitpunkt 96h in der vorliegenden Studie sich möglicherweise über einen Zusammenhang zum Messzeitpunkt erklärt. Ergänzend könnte für diesen Zeitraum auch eine Verfeinerung der 
Messintervalle, ähnlich dem Ansatz von Lunshof et al, sinnvoll sein, um den Verlauf der AAC engmaschiger nachvollziehen zu können (vgl. Lunshof et al 2005). Die Durchführbarkeit auf Grund des Risikos einer möglichen, vorzeitigen Entbindung bei einem Kollektiv mit drohender Frühgeburt, die Verfügbarkeit durch den meist zeitlich begrenzten, stationären Aufenthalt der Schwangeren nach Betamethasongabe, sowie die Akzeptanz der häufigeren Messungen durch die Patienten, stellen hierbei limitierende Faktoren dar.

In einigen Studien konnte ein initialer Anstieg der fetalen Herzratenvariabilität nach der Gabe von Glucocorticoiden nachgewiesen werden. Auch in der vorliegenden Studie konnte, sowohl für die STV als auch für die AAC im CTG und im fetalen EKG, ein tendenzieller Anstieg der Mittelwerte zwischen Oh und 24h unter Betamethasoneinfluss beobachtet werden.

Dawes et al stellten nach Dexamethasongabe einen transienten Anstieg der STV am Tag nach der Gabe, von einem Mittelwert von 6,4 auf 9,8, fest. Bei Präeklampsie oder IUGR, assoziiert mit einem auffälligen Flussprofil der Umbilikalarterien, war dieser Anstieg jedoch geringer ausgeprägt (vgl. Dawes 1994). Mulder et al fanden nach der Gabe von Dexamethason ebenfalls einen signifikanten Anstieg der STV nach 24 Stunden. In Folge von Betamethasongabe waren lediglich die Abnahme der STV 48 und 72 Stunden nach der Gabe signifikant (vgl. Mulder et al 1997).Subtil et al verglichen ebenfalls Beta- und Dexamethason mit einander und konnten für beide Präparate ähnliche Tendenzen aufzeigen: für die STV zeigte sich innerhalb von 32 Stunden nach der Gabe von Betamethason ein Anstieg, zwischen 56 und 80 Stunden eine Reduktion und schließlich eine erneute Annährung in Richtung des Ausgangswertes (vgl. Subtil et al 2003).

Mulder et al beschreiben zusammenfassend die Reaktion der fetalen Herzfrequenzvariabilität auf Betamethason als zweiphasig: die erste Phase zwischen null und 24 Stunden nach der ersten Dosis führt zu einem Anstieg der fetalen Herzfrequenzvariabilität, die zweite Phase ist gekennzeichnet durch einen Abfall der fetalen Herzfrequenzvariabilität nach mehr als 24 Stunden. Diese abfallende Tendenz in der zweiten Phase beschreiben Mulder et al für Dexame- 
thason als weniger deutlich im Vergleich zu Betamethason (vgl. Mulder et al 2009).

In der Untersuchung von Lunshof et al waren die Veränderungen der STV und LTV nach sechs bis zwölf Stunden lediglich bei Feten zwischen 29 und 33 SSW signifikant, für Feten zwischen 25-28 SSW hingegen nicht (vgl. Lunshof et al 2005). Den Zusammenhang zwischen der Wirkung von Betamethason in Abhängigkeit vom Gestationsalter untersuchten auch Mulder et al. Sie konnten für verschiedene Parameter wie beispielsweise die fetale Herzfrequenz, Atem- und Körperbewegungen, nicht jedoch für die fetale Herzfrequenzvariabilität (LTV), ein altersbezogenes Ansprechen auf Betamethason zeigen (vgl. Mulder et al 2004).

Entsprechend der zwei beschrieben Phasen des Ansprechens der fetalen Herzfrequenzvariabilität auf Betamethason werden zwei zu Grunde liegende Mechanismen angenommen (vgl. Mulder et al 2009). Zum einen die direkte und indirekte vasokonstriktorische Wirkung der Glucocorticoide (vgl. Koenen et al 2002, vgl. Derks 1997) welche vor allem im Zeitraum zwischen Oh und $24 \mathrm{~h}$ vorherrschend ist (vgl. Mulder et al 2009). Durch Barorezeptoren wird reflektorisch durch den auf die Vasokonstriktion folgenden Blutdruckanstieg, eine Abnahme der fetalen Herzfrequenz verursacht (vgl. Mulder et al 2004, vgl. Bennet et al 1999). Diese korreliert invers mit der Herzfrequenzvariabilität (vgl. Mulder et al 2004, vgl. Nijhuis et al 1998).

Die Veränderungen über 24h hinaus sehen Mulder et al vor allem durch die zentrale Wirkweise der Glucocorticoide verursacht (vgl. Mulder et al 2009). Glucocorticoide üben durch Bindung an Rezeptoren im zentralen Nervensystem ebenfalls Wirkung auf die fetale Herzfrequenz aus (vgl. Lunshof et al 2005, vgl. Mulder et al 2009, vgl. Koenen et al 2002). Glucocorticoidrezeptoren finden sich in verschiedenen Strukturen des limbischen Systems wie dem Hippocampus (vgl. de Kloet et al 1990), sowie verschiedenen Hirnstammkernen (vgl. Mulder et al 2004, vgl. Matthews 2000). In Tierexperimenten konnte gezeigt werden, dass der Großteil der neuroendokrinen Entwicklung, einschließlich der Entwicklung der Glucocorticoid-Rezeptoren in Primaten und Schafen bereits in utero stattfindet (vgl. Matthews SG 2000). 
Koenen et al sowie De Heus et al weisen auf die Bedeutung des physiologischen Anstieges der fetalen Herzfrequenzvariation im Tagesverlauf hin, welchen sie auf das Vorhandensein eines fetalen Tagesrhythmus zurückführen. Durch diesen Rhythmus kommt es demnach zu Veränderungen in der Herzrate, Herzfrequenzvariabilität, Atem- und Körperbewegung im Tagesverlauf mit einem Anstieg der fetalen HRV am Nachmittag. Die maternale Betamethasongabe führt so möglicherweise über eine transiente Aufhebung dieses Tagesrhythmus, zu einem Fehlen des normalerweise zu beobachtenden Anstieges der HRV am Nachmittag (vgl. Koenen et al 2005, de Heus et al 2008). In der vorliegenden Studie richtete sich der Messzeitpunkt Oh, sowie folglich auch der Zeitpunkt der Folgemessungen, nach dem Zeitpunkt der Indikationsstellung zu Lungenreifeinduktion durch den betreuenden Arzt. Im Median lag der Messzeitpunkt in unserer Untersuchung damit um 16:37 Uhr (MEZ).

\subsection{Pharmakologie}

Die Injektion der Betamethason Dosis ist zweigeteilt, es werden 2x12mg im Abstand von $24 \mathrm{~h}$ intramuskulär injiziert. Die erste Injektion erfolgt nach der ersten Messung zum Zeitpunkt Oh. Die zweite Injektion zum Zeitpunkt der 24h Messung. Die biologische Halbwertszeit von Betamethason (Celestan ${ }^{\circledR}$ solubile 4mg, MSD Sharp und Dohme $\mathrm{GmbH}$ ) beträgt $36-54 \mathrm{~h}$ bei einer PlasmaHalbwertszeit von $>=300 \mathrm{~min}$. Das bedeutet, dass es zu einer Kumulation des Medikaments im Körper bei wiederholter Anwendung kommen kann (vgl. Fachinformation Celestan ${ }^{\circledR}$ solubile: http://www.msd.de/produkte/msdprodukte/pdf/celestan-solubile.pdf, Stand Dezember 2014).

Das Ende des Betamethasoneinflusses auf die STV nach 96h lässt sich im Oxford CTG durch eine signifikant hohe Korrelation der Werte nach 96h abbilden. Zuvor zeigt sich eine transiente Aufhebung der hohen Korrelation der Messwerte im Bereich zwischen 24h und 48h. Der Zeitraum zwischen 24h und $48 \mathrm{~h}$ entspricht dem zeitlichen Bereich, in dem die zu erwartende Kortikosteroidkonzentration im mütterlichen sowie fetalen Organismus vorübergehend am höchsten ist. 


\subsection{Statistik}

\subsubsection{Fehlende Werte}

In der vorliegenden Studie kam es bei zwei Teilnehmerinnen zur Entbindung vor erfolgter Messung zum Zeitpunkt $96 \mathrm{~h}$.

Die Daten einer Teilnehmerin konnten auf Grund des Zeitpunktes des Eintritts in die Studie lediglich zur Auswertung der STV im CTG und fetalen EKG jedoch nicht mehr für die Auswertung der AAC mittels PRSA-Technik verwendet werden.

Eine weitere Ursache fehlender Werte dieser Studie ist der Signalverlust in der Messung, in den Kapiteln 4.1.1 „Signalverlust im fetalen EKG“ und 4.1.2 „Signalverlust im CTG“" werden mögliche Ursachen für Signalverluste in beiden Geräten ausführlich diskutiert.

Für das CTG liegen neben den in den oben genannten Kapiteln besprochenen Werten keine weiteren fehlenden Werte vor. Im fetalen EKG zeigen sich neben den im oben genannten Kapitel besprochenen Werte, noch zwei weitere fehlende Werte für die STV und ein weiterer fehlender Wert für die AAC. Die Ursachen hierfür werden im Folgenden, in Zusammenschau mit sämtlichen fehlenden Werten im fetalen EKG, erläutert.

Für die STV im fetalen EKG existieren insgesamt 44 fehlende Messwerte. Diese setzen sich aus den zwei auf Grund von Entbindung fehlenden Werten, 29 Werten mit 100\% Signalverlust (Global- und/oder Analysen-Signalverlust) sowie elf weiteren Werten zusammen, für welche auch keine Angaben über die Höhe eines möglichen Signalverlustes existieren. Für diese elf Werte werden daher im Kapitel 4.1.1. „Signalverlust im fetalen EKG“ die möglichen Ebenen des Informationsverlustes ausführlich erläutert. Des Weiteren gibt es zwei fehlende Werte der STV im fetalen EKG, für die bemerkenswerterweise eine AAC berechnet werden konnte und die daher keiner der bisher genannten Ursachen zugeordnet werden kann. Die Ursache dieser fehlenden STV muss daher auf Ebene der Software des CTG vermutet werden, welche die STV aus den Rohdaten berechnet und speichert. 
Für die AAC im fetalen EKG konnten auf Grund des späteren Eintritts in die Studie vier Daten einer Patientin nicht mehr in die Auswertung mit einbezogen werden. Darüber hinaus existieren 18 weitere fehlende Daten. Diese setzen sich aus zwei auf Grund von Entbindung vor der letzten Messung fehlenden Werten, vier Werten mit 100\% Signalverlust (Global- und/oder AnalysenSignalverlust), sowie ebenfalls den oben genannten elf Werten zusammen, für die auch keine Angaben über die Höhe eines möglichen Signalverlustes existieren. Für diese 11 Werte werden daher im Kapitel 4.1.1. „Signalverlust im fetalen EKG“ mögliche Ebenen und damit Ursachen des Informationsverlustes ausführlich erläutert. Für einen fehlenden Wert der AAC im fetalen EKG existiert jedoch bemerkenswerterweise eine STV. Hier muss die Ursache demnach auf Ebene der Software vermutet werden, welche die Rohdaten analysiert und die Daten speichert.

\subsubsection{Berücksichtigung fehlender Werte im T-Test und den Korrelationen}

Der in der Literatur beschriebene Verlauf der STV unter Betamethasoneinfluss wurde in der vorliegenden Studie anhand der Überprüfung der Mittelwerte mittels T-Test für gepaarte Stichproben verglichen. Entsprechend wurde auch der Verlauf der Mittelwerte der anderen Parameter beschrieben. Auf Grund fehlender Einzelwerte, deren Ursachen im Kapitel 4.4.1 „Fehlende Werte“ ausführlich erläutert werden, weicht der jeweilige Stichprobenumfang teilweise von der Gesamtzahl der 28 Teilnehmerinnen der Studie ab. Für jeden Mittelwertvergleich ist daher der jeweilige Stichprobenumfang als sog. „Sample size“ in der entsprechenden Tabelle aufgeführt. Die Anzahl der vergleichbaren Werte liegt für die STV im CTG zwischen 20 und 24, für die AAC im CTG ist diese mit 19 bis 23 etwas geringer. Im fetalen EKG ergab sich mit zwölf bis 15 für die STV die geringste Anzahl an vergleichbaren Werten. Für die AAC im EKG lag die Anzahl vergleichbarer Werte zwischen 20 und 22.

Auch in der Berechnung der Korrelationen weicht auf Grund fehlender Einzelwerte die Anzahl der letztlich mit einander korrelierten Wertepaare teilweise von der Gesamtzahl der Teilnehmerinnen ab. Die Anzahl der korrelierten Wertepaa- 
re für jede beschriebene Korrelation ist ebenfalls als sog. „Sample size“ in den entsprechenden Tabellen und erläuternden Textabschnitten aufgeführt.

Für die STV im CTG liegt die Anzahl dieser Wertepaare zwischen 21 und 24, für die AAC im CTG zwischen 20 und 23. Für die AAC im EKG liegt die Anzahl der Wertepaare ähnlich hoch zwischen 20 und 22. Die STV im EKG weist mit einer Sample size zwischen 13 und 15 die geringste Anzahl vergleichbarer Wertepaare auf.

Sowohl in den T-Tests als auch in der Korrelation findet sich die geringste Anzahl an vergleichbaren Werten für die STV im fetalen EKG.

\subsubsection{Anzahl der Studienteilnehmerinnen}

Dem Problem der fehlenden Daten durch den unvorhersehbaren Signalverlust, hätte wünschenswerterweise mit einer größeren Anzahl an Studienteilnehmerinnen entgegen gewirkt werden können. Dies zeigte sich jedoch durch die limitierte Verfügbarkeit des gesuchten Patientenkollektives im Zeitraum der Datenerhebung als nicht realisierbar. Die Datenerhebung wurde von ursprünglich einem Jahr auf zwei Jahre verdoppelt. Viele der Patientinnen, welche Betamethason im Klinikum erhielten, kamen für die den Einschluss in die Studie nicht in Frage: Bei einem Großteil war bereits vorab sicher, dass die unmittelbare Entbindung (bis spätestens $48 \mathrm{~h}$ nach Betamethason Erstgabe) bevorsteht. Außerdem war es bei einem weiteren Anteil durch den behandelnden Arzt aus medizinischer Sicht nicht zu vertreten, die Betamethasongabe nach Indikationsstellung bis zum Eintreffen der Doktorandin zu verzögern. Der Studieneinschluss der Patientin verzögerte die Medikamentengabe um ca. zwei bis vier Stunden (die genannte Zeit setzt sich zusammen aus der Information der Doktorandin durch den Dienstarzt, Anfahrt der Doktorandin zur Klinik, Patientenaufklärung, CTG und fetales EKG Aufzeichnung über 40 Minuten vor der Medikamentengabe).

\subsubsection{Wahl des Korrelationskoeffizienten}

Bei der Untersuchung auf das Vorliegen eines linearen Zusammenhangs der Parameter mittels Streudiagramm (Scatterplot), zeigten sich in zwei Fällen deut- 
lich abweichende Einzelwerte. Aufgrund der für diese Fälle nicht sicher anzunehmenden Voraussetzung einer gleichmäßigen Normalverteilung wurde für die gesamte Studie der Reihenkorrelationskoeffizient nach Spearman (Rho) gewählt.

In Abbildung 24 sind die Werte der STV im fetalen EKG zu den Zeitpunkten Oh und $96 \mathrm{~h}$ in einem Streudiagramm dargestellt. Es zeigen sich zwei deutlich von der Regressionsgerade entfernt liegende Einzelwerte in der Verteilung.

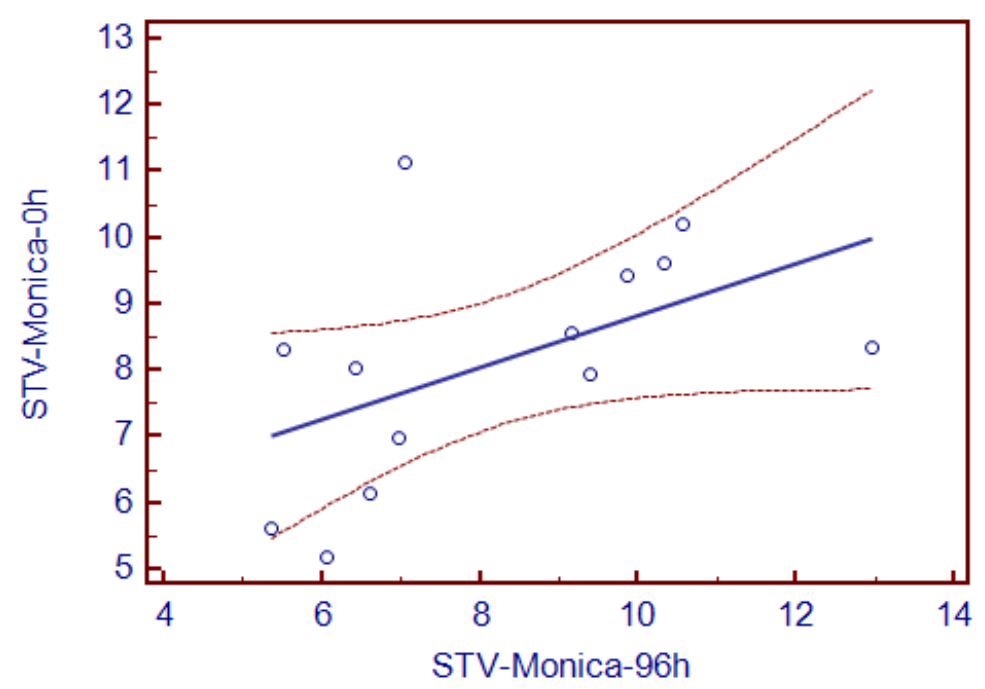

Abb.24: Streudiagramm der Werte der STV im Monica für die Zeitpunkte Oh und 96h, durchgezogene Linie $=$ Regressionsgerade, gestrichelte Linien=95\% Konfidenzintervall.

Ebenfalls stark von der Gesamtverteilung abweichende Einzelwerte fanden sich in der Untersuchung der Werte der STV und AAC im CTG zum Zeitpunkt Oh. Abbildung 25 zeigt das Streudiagramm der Werteverteilung um die Regressionsgerade, deutlich zu erkennen weicht ein Einzelwert stark von der Regressionsgeraden $a b$. 


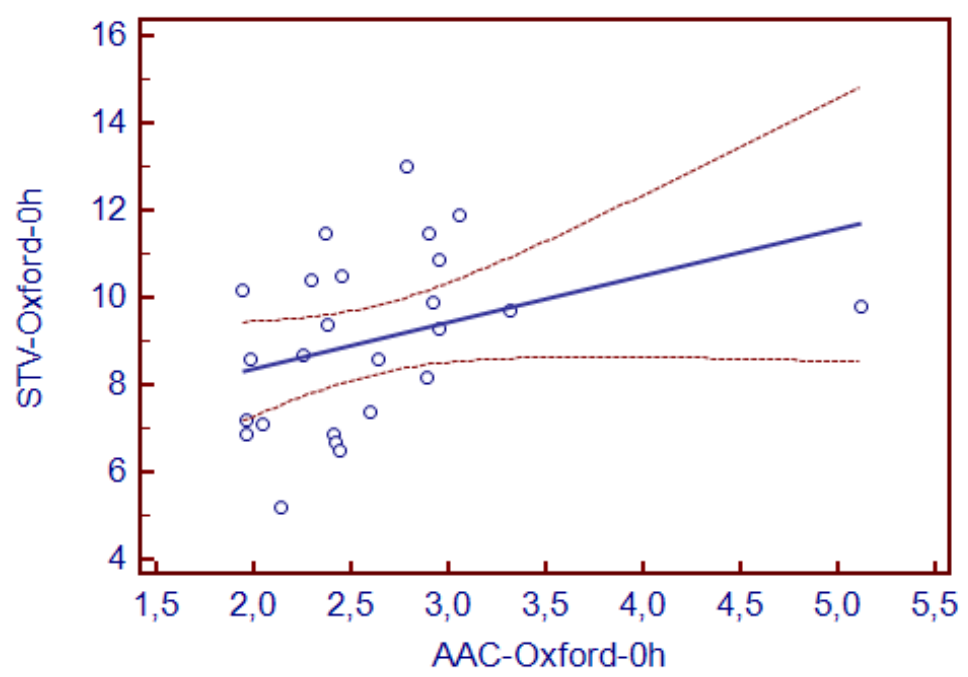

Abb. 25: Streudiagramm der Werte der STV sowie AAC im Oxford zum Zeitpunkt Oh, durchgezogene Linie $=$ Regressionsgerade, gestrichelte Linien=95\%-Konfidenzintervall 


\section{$5 \quad$ Zusammenfassung und Ausblick}

Ziel der Studie:

Zur Überwachung des fetalen Zustands verwendete Parameter, wie die Kurzzeitvariation der fetalen Herzfrequenz, werden durch die Gabe von Kortikosteroiden vorübergehend beeinflusst. Durch die Signalverabeitungsmethode PRSA (engl., phase-rectified signal averaging, phasengleichgerichtete Signalmittelung) konnte ein neuer Parameter zur Analyse der fetalen Herzfrequenzvariabilität entwickelt werden, die sog. Akzelerationskapazität (vgl. Huhn et al 2011, Lobmaier et al 2012). Lobmaier und Huhn et al konnten bereits für IUGR-Feten zeigen, dass die Akzelerationskapazität als Überwachungsparameter der fetalen Herzfrequenz im Kardiotokogramm (CTG) eine genauere und zuverlässigere Unterscheidung zwischen normalgewichtigen Feten und IUGR-Feten ermöglicht, als die Kurzzeitvariation (vgl. Lobmaier et al 2012). In der Erwachsenenkardiologie konnte mit Hilfe der PRSA-Signalverarbeitung für das EKG bereits in Form der Dezelerationskapazität ein neuer Parameter definiert werden, der dem bis dahin bestehenden Goldstandard in der Postinfarktdiagnostik überlegen ist (vgl. Bauer et al 2006b).

In der vorliegenden Arbeit soll das Verhalten des Parameters Akzelerationskapazität im Vergleich zum Verhalten der Kurzzeitvariation unter Betamethasoneinfluss zur Lungenreifeinduktion bei drohender Frühgeburt untersucht werden. Die fetale Herzaktion wird hierzu gleichzeitig mittels CTG und abdominellem, fetalen EKG registriert. Auch diese beiden Methoden werden miteinander verglichen.

Durchführung/ Methodik: In einer prospektiven Studie wurden von August 2010 bis Mai 2012 bei 28 Patientinnen der Frauenklinik des Klinikum rechts der Isar zwischen der 24. und 34. SSW, welche Betamethason (Celestan $\AA$ ) solubile 4mg, MSD Sharp und Dohme GmbH, Dosierung: 2x12mg im Abstand von 24h) zur Lungenreifebehandlung bei drohender Frühgeburt erhielten, Aufzeichnun- 
gen der fetalen Herzaktion mittels computerisiertem CTG von sowie zeitgleich mit dem fetalem EKG durchgeführt.

Die Aufzeichnungen dauerten je 40 Minuten und erfolgten vor der Gabe (Zeitpunkt 0), 24 Stunden, 48 Stunden sowie 96 Stunden nach der ersten Gabe des Medikaments. Die Messungen wurden für die jeweilige Patientin stets zur selben Tageszeit durchgeführt. Im Median lag der Messzeitpunkt am Nachmittag um ca.17:00 Uhr. Die CTG Aufzeichnungen erfolgte mit dem Gerät Sonicaid Team Care der Firma HNE (Huntleigh Nesbit Evans Healthcare GmbH, Cardiff, UK). Das fetale Elektrokardiogramm wurde mit dem Gerät AN24 ${ }^{\mathrm{TM}}$ der Firma Monia Healthcare Ltd. Nottingham, UK aufgezeichnet. Es wurden hierfür fünf nicht-invasive, selbstklebende Elektroden in standardisierter Position auf den Bauch der Mutter aufgebracht.

Zur Auswertung der Daten für die Dawes Redman Analyse wurde für das CTG die Software Sonicaid Fetal Care (HNE Huntleigh Nesbit Evans Healthcare $\mathrm{GmbH}$ ) verwendet, für das fetale EKG die Software Monica DK 1.5b (Monica Healthcare). Zur Bearbeitung der CTG Rohdaten mittels PRSA, wurde das Programm LibRASCH verwendet. Ein Programm, das zur Analyse und Verarbeitung von biologischen Signalen entwickelt wurde (vgl. Schneider et al 2004, vgl. www.librasch.org).

Ergebnisse: Im CTG zeigt sich eine signifikante, hohe Korrelation der Werte der Kurzzeitvariation vor Kortisongabe (Zeitpunkt Oh) und 96h danach. Dies ließ sich ebenso für den neuen Parameter Akzelerationskapazität im CTG zeigen (Korrelation der Messwerte der STV zwischen 0h und 96h im CTG: SpearmanKorrelationskoeffizient $=0,69, p=0,0005,95 \% \mathrm{Cl}$ : [0,37-0,87], Korrelation der Messwerte der AAC zwischen 0h und 96h im CTG: SpearmanKorrelationskoeffizient $=0,64, p=0,002,95 \% \mathrm{Cl}=[0,28-0,84])$. Zwischen $24 \mathrm{~h}$ und 48h kam es für beide Parameter im CTG zu einer transienten Aufhebung dieser hohen, signifikanten Korrelation ihrer Ausgangsmesswerte zu den Werten in diesem Messzeitraum. Sowohl für die Kurzzeitvariation, als auch für die Akzelerationskapazität im CTG konnte in Form dieser passager verminderten Korrelation zwischen $24 \mathrm{~h}$ und $48 \mathrm{~h}$, der Steroideinfluss in diesem Zeitraum abgebildet werden. Nach 24 Stunden zeigte sich ein tendenzieller Anstieg, nach 48 Stun- 
den ein Abfall der Messwerte. Ebenfalls konnte so gezeigt werden, dass nach 96h für beide Parameter im CTG keine relevante Beeinflussung der Korrelation durch die Steroidgabe mehr besteht.

Bei Erhebung der Rohdaten mit dem fetalen EKG fand sich für die Kurzzeitvariation ebenfalls eine passagere, geringe Korrelation im Zeitraum $24 \mathrm{~h}$ bis $48 \mathrm{~h}$ nach Steroidgabe. Nach 96h lag eine höhere, signifikante Korrelation zu den Ausgangsmesswerten vor (Korrelation der Messwerte der STV zwischen Oh und 96h im fetalen EKG: Spearman-Korrelationskoeffizient (Rho) $=0,67, p=0,01$, $95 \% \mathrm{Cl}=[0,19-0,89])$. Auch für die Kurzzeitvariation im fetalen EKG ist daher anzunehmen, dass nach 96h keine relevante Beeinflussung der Korrelation in Folge der Steroidgabe mehr besteht. Für den Zeitpunkt 48h nach Medikamentengabe ist diese hingegen anzunehmen. Für die Akzelerationskapazität im fetalen EKG zeigte sich ein anderer Verlauf der Korrelation. Zum Zeitpunkt $96 \mathrm{~h}$ lag hier die niedrigste Korrelation zu den Ausgangsmesswerten im Vergleich aller Zeitpunkte vor. Auch der Mittelwert der Akzelerationskapazität im fetalen EKG zeigte zwischen 48h und 96h keine Tendenz zur Rückkehr auf das Ausgangsniveau (Korrelation der Messwerte der AAC im fetalen EKG zwischen Oh und 96h: Spearman Korrelationskoeffizient $=0,13, p=0,56,95 \% \mathrm{Cl}=[(-0,34)$ 0,54)], mittlere Abnahme der Akzelerationskapazität zwischen 48h und $96 \mathrm{~h}$ im Vergleich der Mittelwerte mittels T-Test: $(-0,3)$ spm, $p=0,18)$.

Der globale Signalverlust im fetalen EKG lag im Mittel bei 33,32\%, der analysenbezogene Signalverlust lag im Mittel mit 58,7\% noch höher. Im Vergleich mit dem Signalverlust im CTG von $12 \%$ ist dies mehr als doppelt so hoch.

\section{Diskussion:}

Die in dieser Studie beschriebenen Korrelationen der Werte im fetalen EKG, insbesondere der Akzelerationskapazität, müssen unter Berücksichtigung des hohen Signalverlustes innerhalb des Gerätes betrachtet werden.

Die Ursachen für den Signalverlust im fetalen EKG können auf mehreren Ebenen liegen, welche den mütterlichen und fetalen Organismus, das portable Gerät selbst und die Software umfassen. Einen wichtigen Punkt stellt, im Unterschied zur Rohdatenerhebung mittels CTG, bei dem der Untersucher während 
der Aufzeichnung Auskunft über die Höhe des Signalverlustes erhält, die erst nach erfolgter Aufzeichnung (bei Übertragung der Rohdaten auf den Computer) ersichtliche Höhe des Signalverlustes dar. In künftigen Studien könnte diesem Problem beispielsweise durch die Übertragung der registrierten Signale mittels WLAN auf den Computer, parallel zur Aufzeichnung, entgegen gewirkt werden. Darüber hinaus könnte durch die Auswahl eines Patientenkollektives mit Feten in fortgeschrittenerem Gestationsalter für künftige Studien die das fetale EKG verwenden, dem Problem der hohen elektrischen Isolation des Feten durch die Vernix caseosa, entgegengewirkt werden.

Das abweichende Verhalten der Korrelation der Ausgangswerte zu den Werten zum Zeitpunkt 96h für die Akzelerationskapazität im fetalen EKG ist am ehesten durch den hohen Signalverlust bei Aufzeichnungen mit dem fetalen EKG zu erklären. Bei einem durchschnittlichen Signalverlust von über $50 \%$ ist das fetale EKG momentan keine geeignete Methode für Herzfrequenzanalysen in der 30. SSW, welche in der vorliegenden Studie das mittlere Gestationsalter darstellte. Dies zeigt sich auch an der teilweise nicht-signifikanten Korrelation der untersuchten Parameter Kurzzeitvariation und Akzelerationskapazität innerhalb der EKG Aufzeichnungen. Im CTG hingegen war zu jedem Messzeitpunkt eine signifikante Korrelation der untersuchten Parameter gegeben.

Das Problem des hohen Signalverlustes als Quelle fehlender Messwerte in der Auswertung, hätte wünschenswerterweise durch eine höhere Anzahl an Studienteilnehmerinnen abgemildert werden können. Leider zeigte sich die Menge der in Frage kommenden Teilnehmerinnen im durchgeführten Studienzeitraum auf 28 begrenzt.

Da die PRSA-Technik ebenfalls nur "Offline" zur Verfügung steht, ist die Anwendung der AAC zur Beurteilung der fetalen Herzfrequenzvariabilität im klinischen Alltag momentan noch nicht realisierbar. Die Entwicklung einer OnlineAnalyse befindet sich derzeit in Vorbereitung.

\section{Ausblick:}

Lobmaier et al zeigten, dass die Akzelerationskapazität in der Überwachung der Herzfrequenzvariabilität von IUGR-Feten im CTG dem etablierten Parameter 
Kurzzeitvariation überlegen ist (vgl. Lobmaier et al 2012). In dieser Studie wurde die Auswirkung der Gabe von Betamethason zur Induktion der Lungenreifung auf die Akzelerationskapazität an einem Kollektiv von Feten zwischen der 24. und 34. SSW untersucht. Es konnte gezeigt werden, dass 96 Stunden nach erfolgter Steroidgabe, keine relevante Beeinflussung der Korrelation der Ausgangsmesswerte der Akzelerationskapazität zu den Werten zum Zeitpunkt 96h mehr vorliegt. Die Akzelerationskapazität ist also genauso wie die STV 96 Stunden nach Betamethasongabe wieder aussagekräftig.

Als nächster Schritt sollte sich in zukünftigen Studien die Untersuchung der Korrelation der Akzelerationskapazität im CTG mit dem Outcome des Kindes bei bestimmten Erkrankungen, wie etwa Gestationsdiabetes oder maternaler $\mathrm{Hy}$ pertonie, anschließen. So könnte die Aussagekraft der Akzelerationskapazität bezüglich des fetalen Wohlbefindens in utero validiert werden. Des Weiteren könnten Langzeitstudien in diesen Kollektiven durchgeführt werden, um zu untersuchen, ob Feten mit einer eingeschränkten Akzelerationskapazität, also einer Beeinträchtigung des fetalen autonomen Nervensystems, auch ein verändertes Langzeitoutcome im Sinne der fetalen Programmierung aufweisen.

Mit der Akzelerationskapazität kann so zukünftig ein neuer Parameter der fetalen Herzfrequenzvariabilität für die klinischen Anwendung zur Verfügung stehen, welcher über die IUGR-Diagnostik hinausgehend, in einer Vielzahl geburtshilflicher Fragestellungen die Beurteilung des fetalen Zustandes verbessern und die Entscheidungsfindung unterstützen kann.

In der Erwachsenenkardiologie konnte mit Hilfe der PRSA-Signalverarbeitung für das EKG bereits in Form der Dezelerationskapazität ein neuer Parameter definiert werden, der dem bis dahin bestehenden Goldstandard in der Postinfarktdiagnostik überlegen ist (vgl. Bauer et al 2006b). Durch die Anwendung der PRSA-Technik auf das fetale EKG könnten entsprechend weitere, diagnostische Fortschritte auch in der Geburtshilfe erzielt werden.

In der vorliegenden Studie konnte bereits der Einfluss von Betamethason auf die Kurzzeitvariabilität im fetalen EKG anhand der Korrelation der Messwerte im zeitlichen Verlauf beschrieben werden und mit dem Werteverlauf im CTG verglichen werden. 


\section{Literaturverzeichnis}

1. ACOG (American Congress of Obstetricians and Gynecologists) Committee Opinion No.475: Antenatal corticosteroid therapy for fetal maturation. Obstetrics and Gynecology 117 (2011) 422-424

2. Akselrod S, Gordon D, Ubel FA, Shannon DC, Berger AC, Cohen RJ. Power spectrum analysis of heart rate fluctuation: a quantitative probe of beatto-beat cardiovascular control.

Science 213 (1981) 220-222

3. AWMF-Leitlinien der deutschen Gesellschaft für Gynäkologie und Geburtshilfe: Antenatale Kortikosteroide zur Lungenreifung (ACS) http://www.awmf.org/uploads/tx_szleitlinien/015069_S1_Antenatale_Kortikosteroide_zur_Lungenreifung_ACS_07-2009_07-2014.pdf (letzter Zugriff: Januar 2014, Leitlinien derzeit in Überarbeitung)

4. AWMF-Leitlinien der deutschen Gesellschaft für Gynäkologie und Geburtshilfe, Arbeitsgemeinschaft Materno-fetale Medizin (AGMFM), Deutsche Gesellschaft für Pränatal und Geburtsmedizin (DGPG), Deutsche Gesellschaft für Perinatale Medizin(DGPM):

Anwendung des CTG während Schwangerschaft und Geburt http://www.awmf.org/uploads/tx_szleitlinien/015-

036I_S1_CTG_Schwangerschaft_Geburt_2014-06.pdf (letzter Zugriff: Dezember 2014)

5. Assali NS, Brinkman CR 3rd, Woods JR Jr., Dandavino A, Nuwayhid B Development of neurohumoral control of fetal, neonatal, and adult cardiovascular functions.

American Journal of Obstetrica and Gynecology 129 (1977) 748-59

6. Avery ME, Mead J.

Surface properties in relation to atelectasis and hyaline membrane disease. American Journal of Diseases of Children 97 (1959) 517-523 
7. Ballard PL, Ballard RA.

Scientific basis and therapeutic regimens for use of antenatal glucocorticoids American journal of obstetrics and gynaecology 173 (1995) 254-262

8. Banks BA, Cnaan A, Morgan MA, Parer JT, Merrill JD, Ballard PL, Ballard RA. Multiple courses of antenatal corticosteroids and outcome of premature neonates. North American Thyrotropin-Releasing Hormone Study Group American Journal of Obstetrics and Gynecology 181(1999) 709-17

9. Baud O, Foix-L'Helias L, Kaminski M, Audibert F, Jarreau PH, Papiernik E, Huon C, Lepercq J, Dehan M, Lacaze-Masmonteil T.

Antenatal glucocorticoid treatment and cystic periventricular leukomalacia in very premature infants.

New England Journal of Medicine 341 (1999) 1190-6

10. Bauer 2006a:

Bauer A., Kantelhardt J.W., Bunde A., Barthel P., Schneider R ., Malik M., Schmidt $G$. Phase-rectified signal averaging detects quasi-periodicities in nonstationary data, Physica A 364 (2006) 423-434

11. Bauer 2006b:

Bauer A, Kantelhardt JW, Barthel P, Schneider R, Mäkikallio T, Ulm K, Hnatkova K, Schömig A, Huikuri H, Bunde A, Malik M, Schmidt G.

Deceleration capacity of heart rate as a predictor of mortality after myocardial infarction: cohort study. Lancet 367 (2006) 1674-1681

12. Bennet L, Kozuma S, McGarrigle HH, Hanson MA.

Teporal changes in fetal cardiovascular, behavioural, metabolic and endocrine responses to maternally administered dexamethasone in the late gestation fetal sheep. British Journal of Obstetrics and Gynaecology 106 (1999) 331-339

13. Blencowe H, Cousens S, Oestergaard MZ, Chou D, Moller AB, Narwal R, Adler A, Vera Garcia C, Rohde S, Say L, Lawn JE. 
National, regional and worldwide estimates of preterm birth rates in the year 2010 with time trends since 1990 for selected countries: a systematic analysis and implications. Lancet 379 (2012) 2162-2172

14. Bolt RJ, van Weissenbruch MM, Lafeber HN, Delemarre-van de Waal HA. Glucocorticoids and Lung Development in the Fetus and Preterm Infant Pediatric Pulmonology 32 (2001) 76-91

15. Bonanno C., Wapner RJ.

Antenatal corticosteroid treatment: what's happened since Drs Liggins and Howie? American Journal of Obstetrics and Gynecology 200 (2009) 448-457

16. BQS Bundesauswertung 2008 Leistungsbereich Geburtshilfe (Institut für Qualität und Patientensicherheit) http://www.bqsoutcome.de/2008/ergebnisse/leistungsbereiche/geburtshilfe/index_html (letzter Zugriff: Dezember 2014)

17. Brownfoot FC, Crowther CA, Middleton P.

Different corticosteroids and regimens for accelerating fetal lung maturation for women at risk of preterm birth.

The Cochrane Database of Systematic Reviews 4 (2008) Art.No:CD006764

18. Crowley PA, Chalmers I, Keirse MJ.

The effects of corticosteroid administration before preterm delivery: an overview of the evidence from controlled trials.

British Journal of Obstetrics and Gynaecology 97 (1990) 11-25

19. Crowley PA

Antenatal corticosteroid therapy: a meta-analysis of the randomized trials, 1972 to 1994

American Journal of Obstetrics and Gynecology 173 (1995) 322-335

20. Crowther 2007a: Crowther CA, Harding JE Repeat doses of prenatal corticosteroids for women at risk of preterm birth for preventing neonatal respiratory disease. 
The Cochrane Database of Systematic Reviews 3(2007) Art.no: CD003935

21. Crowther 2007b /ACTORDS Study Group:

Crowther CA, Doyle LW, Haslam RR, Hiller JE, Harding JE, Robinson JS; ACTORDS Study Group.

Outcomes at 2 years of age after repeat doses of antenatal corticosteroids. New England Journal of Medicine 357 (2007) 1179-1189

22. Cohlen BJ, Stigter RH, Derks JB, Mulder EJ, Visser GH

Absence of significant hemodynamic changes in the fetus following maternal betamethasone administration

Ultrasound in Obstetrics and Gynecology 8 (1996) 252-255

23. David M, Hirsch M, Karin J, Toledo E, Akselrod S.

An estimate of fetal autonomic state by time-frequency analysis of fetal heartrate variability. Journal of Applied Physiology 102 (2007) 1057-1064

24. Dawes GS, Houghton CR, Redman CWG

Baseline in human fetal heart-rate records.

British Journal of Obstetrics and Gynaecology 89 (1982) 270-275

25. Dawes G.S., Moulden M., Redman C.W.G.

The advantages of computerized fetal heart rate analysis

Journal of Perinatal Medicine 19 (1991) 39-45

26. Dawes 1992a:

Dawes GS, Lobb M, Moulden M, Redman CW, Wheeler T.

Antenatal cardiotocogram quality and interpretation using computers

British Journal of Obstetrics and Gynaecology 99 (1992) 791-797

27. Dawes 1992b:

Dawes GS, Moulden M, Redman CW.

Short-term fetal heart rate variation, decelerations, and umbilical flow velocity waveforms before labor.

Obstetrics and Gynecology 80 (1992) 673-678 
28. Dawes GS, Serra-Serra V, Moulden M, Redman CW.

Dexamethasone and fetal heart rate variation.

British Journal of Obstetrics and Gynaecology 101 (1994) 675-679

29. de Heus R, Mulder EJ, Derks JB, Koenen SV, Visser GH.

Differential effects of betamethasone on the fetus between morning and afternoon recordings.

The Journal of maternal- fetal and neonatal medicine 21 (2008) 549-554

30. de Kloet ER, Reul JM, Sutanto W.

Corticosteroids and the brain.

The Journal of steroid biochemistry and molecular biology 37 (1990) 387-394

31. Derks JB, Mulder EJ, Visser GH.

The effects of maternal betamethasone administration on the fetus.

British Journal of Obstetrics and Gynaecology 102 (1995) 40-46

32. Derks JB, Giussani DA, Jenkins SL, Wentworth RA, Visser GH, Padbury JF, Nathanielsz PW.

A comparative study of cardiovascular, endocrine and behavioural effects of bet amethasone anddexamethasone administration to fetal sheep.

The Journal of Physiology 499 (1997) 217-226

33. Dessens AB, Haas HS, Koppe JG.

Twenty-year follow-up of antenatal corticosteroid treatment.

Pediatrics. 105 (2000) E77

34. Devoe L, Golde S, Kilman Y, Morton D, Shea K, Waller J.

A comparison of visual analyses of intrapartum fetal heart rate tracings according to the new national institute of child health and human development guidelines with computer analyses by an automated fetal heart rate monitoring system.

American Journal of Obstetrics and Gynecology 183 (2000) 361-366 
35. Dunlop SA, Archer MA, Quinlivan JA, Beazley LD, Newnham JP. Repeated prenatal corticosteroids delay myelination in the ovine central nervous system.

Journal of Maternal and Fetal Medicine 6 (1997) 309-313

36. Elimian A, Garry D, Figueroa R, Spitzer A, Wiencek V, Quirk JG.

Antenatal betamethasone compared with dexamethasone (betacode trial): a randomized controlled trial.

Obstetrics and Gynecology 110( 2007) 26-30

37. Fachinformation Celestan® solubile http://www.msd.de/produkte/msd-produkte/pdf/celestan-solubile.pdf (letzter Zugriff: Dezember 2014)

38. Feldman DM, Carbone J, Belden L, Borgida AF, Herson V.

Betamethasone vs dexamethasone for the prevention of morbidity in very-lowbirthweight neonates.

American Journal of Obstetrics and Gynecology 197(2007) 284.e1-4

39. Fisher JE, Smith RS, Lagrandeur R, Lorenz RP.

Gestational diabetes mellitus in women receiving beta-

adrenergics and corticosteroids for threatenedpreterm delivery.

Obstetrics and Gynecology 90(1997) 880-883

40. French NP, Hagan R, Evans SF, Mullan A, Newnham JP.

Repeated antenatal corticosteroids: effects on cerebral palsy and childhood behavior.

American Journal of Obstetrics and Gynecology 190 (2004) 588-95

41. Galazios G, Tripsianis G, Tsikouras P, Koutlaki N, Liberis V.

Fetal distress evaluation using and analyzing the variables of antepar-

tum computerized cardiotocography.

Archives of Gynecology and Obstetrics 281 (2010) 229-233 
42. Gnirs J, Schneider K.T.M. Die Geburtsüberwachung. In: „Die Geburtshilfe“ Schneider H., Husslein P., Schneider K.T.M. (Hrsg.) Springer- Verlag Berlin Heidelberg, 2011, 4.Auflage, S. 724-767

43. Graatsma EM, Mulder EJ, Vasak B, Lobmaier SM, Pildner von Steinburg S, Schneider KT, Schmidt G, Visser GH. Average acceleration and deceleration capacity of fetal heart rate in normal pregnancy and in pregnancies complicated by fetal growth restriction. The journal of maternal-fetal and Neonatal Medicine 12 (2012) 2517-2522

44. Haynes LE, Griffiths MR, Hyde RE, Barber DJ, Mitchell IJ.

Dexamethasone induces limited apoptosis and extensive sublethal damage to specific subregions of the striatum and hippocampus: implications for mood disorders.

Neuroscience 104 (2001) 57-69

45. Hon EH, Lee ST.

Electronic Evaluation of the fetal heart rate. VIII. Patterns preceding fetal death, further observations.

American Journal of Obstetrics and Gynecology 87 (1963) 814-826.

46. Huang 1999

Huang WL, Beazley LD, Quinlivan JA, Evans SF, Nenham JP, Dunlop SA. Effect of corticosteroids on brain growth in fetal sheep.

Obstetrics \& Gynecology 94 (1999) 213-218

47. Huang et al 2001a:

Huang WL, Harper CG, Evans SF, Newnham JP, Dunlop SA.

Repeated prenatal corticosteroid administration delays myelination of the corpus callosum in fetal sheep.

International Journal of Developmental Neuroscience 19 (2001) 415-425

48. Huang et al 2001b:

Huang WL, Harper CG, Evans SF, Newnham JP, Dunlop SA.

Repeated prenatal corticosteroid administration delays astrocyte and capillary tight junction maturation in fetal sheep. 
International Journal of Developmental Neuroscience 19 (2001) 487-493

49. Huhn E.A.

Phasengleichgerichtete Signalmittelung der Kardiotokographie bei intrauteriner Wachstumsretardierung.

Dissertation des Fachbereichs Medizin der Technischen Universität München (2006)

50. Huhn EA, Lobmaier S, Fischer T, Schneider R, Bauer A, Schneider

KT, Schmidt G, New computerized fetal heart rate analysis for surveillance of intrauterine growth restriction.

Prenatal diagnosis 31 (2011) 509-514

51. Jensen EC, Bennet L, Guild SJ, Booth LC, Stewart J, Gunn AJ.

The role of the neural sympathetic and parasympathetic systems

in diurnal and sleep state-related cardiovascular rhythms in the late-

gestation ovine fetus.

American Journal of Physiology. Regulatory, integrative and comparative physiol. 297 (2009) R998-R1008.

52. Ikegami M, Jibe $A H$, Newnham J, Polk DH, Willet KE, Sly P.

Repetitive prenatal glucocorticoids improve lung function and decrease growth in preterm lambs.

American Journal of Respiratory and critical care medicine 156 (1997) 178-184

53. Jobe $A H$, Soll RF.

Choice and dose of corticosteroid for antenatal treatments.

American Journal of Obstetrics and Gynecology 190 (2004) 878-881

54. Keith RDF, Beckley S, Garibaldi JM, Westgate JA, Ifeachor EC, Greene KR A multicentre comparitive study of 17 experts and an intelligent computer system for managing labour using the cardiotocogram. British Journal of Obstetrics and Gynaecology 102 (1995) 688-700

55. Kelly MK, Schneider EP, Petrikovsky BM, Lesser ML.

Effect of antenatal steroid administration on the fetal biophysical profile. 
Journal of Clinical Ultrasound 28 (2000) 224-226

56. Koenen SV, Mecenas CA, Smith GS, Jenkins S, Nathanielsz PW.

Effects of maternal betamethasone administration on fetal and maternal blood pressure and heart rate in the baboon at 0.7 of gestation.

American Journal of Obstetrics and Gynecology 186 (2002) 812-817

57. Koenen SV, Mulder EJ, Wijnberger LD, Visser GH.

Transient loss of the diurnal rhythms of fetal movements, heart rate, and its variation after maternal betamethasone administration.

Pediatric Research 57 (2005) 662-666

58. Lee CY, Di Loreto PC, O'Lane JM.

A study of fetal heart rate acceleration patterns.

Obstetrics and Gynecology 45 (1975) 142-146

59. Lee BH, Stoll BJ, McDonald SA, Higgins RD; National Institute of Child Health and Human Development Neonatal Research Network.

Adverse neonatal outcomes associated with antenatal dexamethasone versus antenatal betamethasone. Pediatrics 117 (2006) 1503-1510

60. Lee BH, Stoll BJ, McDonald SA, Higgins RD

Neurodevelopmental outcomes of extremely low birth weight infants exposed prenatally to dexamethasone versus betamethasone.

Pediatrics 121 (2008) 289-296

61. Liggins GC.

Premature delivery of foetal lambs infused with corticosteroids.

Journal of Endocrinology 45 (1969) 515-523

62. Liggins GC, Howie RN.

A controlled trial of antepartum glucocorticoid treatment for prevention of the respiratory distress syndrome in premature infants.

Pediatrics 50 (1972) 515-525 


\section{Lobmaier SM}

Neue Methode zur Überwachung bei intrauteriner Wachstumsrestriktion: Phasengleichgerichtete Signalmittelung der fetalen Herzfrequenz

Dissertation des Fachbereichs Medizin der Technischen Universität München (2012)

64. Lobmaier SM, Huhn EA, Pildner von Steinburg S, Müller A, Schuster T, Ortiz JU, Schmidt G, Schneider KT.

Phase-rectified signal averaging as a new method for surveillance of growth restricted fetuses.

The Journal of materal-fetal and neonatal medicine 12 (2012) 2523-2528

65. Lunshof MS, Boer K, Wolf H, Koppen S, Velderman JK, Mulder EJ.

Short-term (0-48 h) effects of maternal betamethasone administration on fetal heart rate and its variability.

Pediatric Research 57 (2005) 545-549

66. Manning Frank A. . Kapitel 2, The fetal heart rate- genesis of fetal heart rate patterns. In: Fetal medicine, principles and practice. Appleton \& Lange Verlag Norwalk, Connecticut 1995

67. Mantel R, van Geijn HP, Ververs IA, Copray FJ.

Automated analysis of near-term antepartum fetal heart rate in relation to fetal behavioral states: the Sonicaid System 8000.

American Journal of Obstetrics and Gynecology 165 (1991) 57-65

68. Martin JA, Hamilton BE, Ventura SJ, Osterman MJK, Wilson EC, Mathews TJ Division of Vital Statistics National vital statistics reports 61 (2012) http://www.cdc.gov/nchs/data/nvsr/nvsr61/nvsr61_01.pdf Letzter Zugriff Dezember 2014)

69. Matthews SG.

Antenatal glucocorticoids and programming of the developing CNS. Pediatric Research 47 (2000) 291-300 
70. Monica Healthcare AN24 ${ }^{\mathrm{TM}}$ Reference Operator Manual, 100-TF-006 Revision I, Monica Healthcare Ltd. 2007-2009, www.monicahealthcare.com , letzter Zugriff: Februar 2010

71. Mulder EJ, Derks JB, Visser GH

Antenatal corticosteroid therapy and fetal behaviour: a randomised study of the effects of betamethasone and dexamethasone.

British Journal of Obstetrics and Gynaecology 104 (1997) 1239-1247

72. Mulder EJ, Koenen SV, Blom I, Visser GH.

The effects of antenatal betamethasone administration on fetal heart rate and $b$ ehaviour depend ongestational age.

Development 76 (2004) 65-77

73. Mulder EJ, de Heus R, Visser GH.

Antenatal corticosteroid therapy: short-term effects on fetal behavior and hemodynamics.

Seminars in fetal and neonatal Medicine 14 (2009) 151-156

74. Nijhuis J.G., Prechtl H.F., Martin C.B. Jr., Bots R.S.

Are there behavioural states in the human fetus?

Early Human Development 6 (1982) 177-195

75. Nijhuis IJ, ten Hof J, Mulder EJ, Nijhuis JG, Narayan H, Taylor DJ, Wasters P, Visser GH. Numerical fetal heart rate analysis: nomograms, minimal duration of recording and intrafetal consistency

Prenatal and Neonatal Medicine 3 (1998) 314-322

76. Nijhuis IJ, ten Hof J, Mulder EJ, Nijhuis JG, Narayan H, Taylor DJ, Visser GH. Fetal heart rate in relation to its variation in normal and growth retarded fetuses. European Journal of Obstetrics, Gynecology and Reproductive Biology 89 (2000) 27-33

77. Ogunyemi D.

Risk factors for acute pulmonary edema in preterm delivery. 
European Journal of Obstetrics Gynecology and Reproductive Biology 133 (2007) 143-147

78. Ohm J.R., Lüke H.D.

Statistische Signalbeschreibung. In: Signalübertragung: Grundlagen der digitalen und analogen Nachrichtenübertragungssysteme.

Springer -Verlag, Berlin- Heidelberg, 2005, 9.Auflage, S.169-221

79. Pardey J., Moulden M., Redman C.W.G.,

A computer system for the numerical analysis of nonstress tests

American Journal of Obstetrics and Gynecology 186 (2002) 1095-1103

80. Peaceman AM, Bajaj K, Kumar P, Grobman WA.

The interval between a single course of antenatal steroids and delivery and its association with neonatal outcomes.

American Journal of Obstetrics and Gynecology 193 (2005) 1165-1169

81. Peters M, Crowe J, Piéri JF, Quartero H, Hayes-Gill B, James D, Stinstra J, Shakespeare S.

Monitoring the fetal heart non-invasively: a review of methods

Journal of Perinatal Medicine 29 (2001) 408-416

82. Philippe A, Curinier S, Piquier-Perret G, Delabaere A, Acoccebery M, Vélémir L, Niro J, Jacquetin B, Lémery D, Gallot D.

Use of Monica AN24(TM) for fetal monitoring during labour induction Journal de gynécologie, obstétrique et biologie de la reproduction 41 (2012) 194-197

83. Piéri JF, Crowe JA, Hayes-Gill BR, Spencer CJ, Bhogal K, James DK. Compact long-term recorder for the transabdominal foetal and maternal electrocardiogram.

Medical and Biological Engineering and Computing 39 (2001) 118-125

84. Pillai 1990a:

Pillai M, James D. 
The importance of the behavioural state in biophysical assessment of the term human fetus. British journal of obstetrics and gynaecology 97 (1990) 1130-1134

85. Pillai 1990b:

Pillai M, James D.

The development of fetal heart rate patterns during normal pregnancy. Obstetrics and Gynecology 76 (1990) 812-816.

86. Quinlivan JA, Beazley LD, Evans SF, Newnham JP, Dunlop SA.

Retinal maturation is delayed by repeated, but not single, maternal injections of betamethasone in sheep.

Eye 14 (2000) 93-98

87. Quinlivan JA, Beazley LD, Archer M, Evans SF, Newnham JP, Dunlop SA. Repeated prenatal corticosteroids reduce glial fibrillary acidic protein in the ovine central nervous system. Journal of Perinatal Medicine 30 (2002) 209-219

88. Reinhard J, Hatzmann H, Schiermeier S.

Foetal electrocardiography (ECG) is an alternative to Doppler ultrasound cardiotocogram (CTG) for antenatal assessment of fetal well-being- preliminary results.

Zeitschrift für Geburtshilfe und Neonatologie 212 (2008) 226-229

89. Roberts D., Dalziel S.

Antenatal corticosteroids for accelerating fetal lung maturation for women at risk of preterm birth.

The Cochrane Database of Systematic Reviews Issue 3 (2006)

Art. No.: CD004454.

90. Rotmensch 1999a

Rotmensch S, Liberati M, Vishne TH, Celentano C, Ben-Rafael Z, Bellati U.

The effect of betamethasone and dexamethasone on fetal heart rate patterns a nd biophysical activities. A prospective randomized trial.

Acta Obstetricia et Gynecologica Scandinavica 78(1999) 493-500 
91. Rotmensch 1999b :

Rotmensch S, Liberati M, Celentano C, Efrat Z, Bar-Hava I, Kovo M, Golan A, Moravski G, Ben-Rafael Z.

The effect of betamethasone on fetal biophysical activities and Doppler velocimetry of umbilical and middle cerebral arteries.

Acta Obstetricia et Gynecologica Scandinavica 78 (1999) 768-773

92. Rotmensch 1999c:

Rotmensch S, Vishne TH, Celentano C, Dan M, Ben-Rafael Z.

Maternal infectious morbidity following multiple courses of betamethasone.

The Journal of Infecion 39 (1999) 49-54

93. Rotmensch S, Lev S, Kovo M, Efrat Z, Zahavi Z, Lev N, Celentano C, BenRafael Z.

Effect of betamethasone administration on fetal heart rate tracing:

a blinded longitudinal study.

Fetal Diagnosis and Therapy 20 (2005) 371-376

94. Royal College of Obstetricians and Gynaecologists (Green-Top Guideline No.7): Antenatal Corticosteroids to Reduce Neonatal Morbidity and Mortality https://www.rcog.org.uk/globalassets/documents/guidelines/gtg_7.pdf (letzter Zugriff: Dezember 2014)

95. Schleußner E

The prevention, diagnosis and treatment of premature labor.

Deutsches Ärzteblatt International 13 (2013) 227-236

96. Schneider H., Helmer $\mathrm{H}$.

Frühgeburt: Präpartale und Intrapartale Aspekte. In: „Die Geburtshilfe“ Schneider H., Husslein P., Schneider K.T.M., Springer-Verlag, Berlin Heidelberg, 2011, 4. Auflage, S.512-555

97. Schneider K.T.M., Gnirs J.

Antepartalüberwachung. In: „Die Geburtshilfe“ Schneider H., Husslein P., Schneider K.T.M. (Hrsg.) Springer- Verlag Berlin Heidelberg, 2011, 4.Auflage, S.648-680 
98. Schneider R, Bauer A, Barthel P, Schmidt G.

libRASCH: a programming framework for transparent access to physiological signals.

Conference Proceedings, Annual international conference of the IEEE Engineering in Medicine and Biology Society. 5 (2004) 3254 - 3257

99. Senat MV, Minoui S, Multon O, Fernandez H, Frydman R, Ville $Y$

Effect of dexamethasone and betamethasone on fetal heart rate variability in preterm labour: a randomised study.

British Journal of Obstetrics and Gynaecology 105 (1998) 749-755

100. Serra V, Bellver J, Moulden M, Redman CW.

Computerized analysis of normal fetal heart rate pattern

throughout gestation

Ultrasound in Obstetrics and Gynecology 34 (2009) 74-79

101. Street P, Dawes GS, Moulden M, Redman CW.

Short-term variation in abnormal antenatal fetal heart rate records.

American Journal of Obstetrics and Gynecology 165 (1991) 515-523

102. Sloboda DM, Moss TJ, Gurrin LC, Newnham JP, Challis JR.

The effect of prenatal betamethasone administration on postnatal

ovine hypothalamic-pituitary-adrenal function

Journal of Endocrinology 172 (2002) 71-81

103. Smolders-de Haas H, Neuvel J, Schmand B, Treffers PE, Koppe JG, Hoeks J. Physical development and medical history of children who were treated antenatally with corticosteroids to prevent respiratory distress syndrome: a 10- to 12-year follow-up.

Pediatrics 86 (1990) 65-70

104. Stevens P.A.

Das Surfactantsystem. In „Surfactanttherapie. Grundlagen, Diagnostik, The rapie“Wauer R.R. (Hrsg.), Georg Thieme Verlag KG, Stuttgart, 2004, 3.

Auflage, S.11-27 
105. Subtil D, Tiberghien P, Devos P, Therby D, Leclerc G, Vaast P, Puech F. Immediate and delayed effects of antenatal corticosteroids on fetal heart rate:

a randomized trial thatcompares betamethasone acetate and phosphate, betamethasone phosphate, and dexamethasone.

American Journal of Obstetrics and Gynecology 188 (2003) 524-531

106. Sweet D, Bevilacqua G, Carnielli V, Greisen G, Plavka R, Saugstad OD et al. European consensus guidelines on the management of neonatal respire tory distress syndrome.

Journal of Perinatal Medicine 35 (2007) 175-186

107. SQG Bundesauswertung Leistungsbereich Geburtshilfe 2013 https://www.sqg.de/downloads/Bundesauswertungen/2013/bu_Gesamt_16 N1-GEBH_2013.pdf

(letzter Zugriff: Dezember 2014)

108. Task Force of The European Society of Cardiology and The North American Society of Pacing and Electrophysiology Heartrate variability. Standards of measurement, physiological interpretation and clinical use.

European Heart Journal 17 (1996) 354-381

109. Visser GH, Dawes GS, Redman CW. Numerical analysis of the normal human antenatal fetal heart rate.

British Journal of Obstetrics and Gynaecology 88 (1981) 792-802

110. Visser GH, Sadovsky G, Nicolaides KH.

Antepartum heart rate patterns in small-for-gestational-age third-trimester fetuses: correlations with blood gas values obtained at cordocentesis. American Journal of Obstetrics and Gynecology 162 (1990) 698-703

111. Vogel M.

Morphologische Entwicklung der fetalen und neonatalen Lunge. 
In: „Surfactanttherapie. Grundlagen, Diagnostik, Therapie“ Wauer R.R. (Hrsg.), Georg Thieme Verlag KG, Stuttgart, 2004, 3. Auflage, S.3-10

112. Wapner RJ, Sorokin Y, Mele L, Johnson F, Dudley DJ, Spong CY, Peace man AM, Leveno KJ, Malone F, Caritis SN, Mercer B, Harper M, Rouse DJ, Thorp JM, Ramin S, Carpenter MW, Gabbe SG Long-term outcomes after repeat doses of antenatal corticosteroids. New England Journal of Medicine 357 (2007) 1190-1198

113. http://www.librasch.org (letzter Zugriff: Dezember 2014) 


\section{$7 \quad$ Abbildungsverzeichnis}

Abbildung 1: Exemplarische CTG- Aufzeichnung (S.13)

Abbildung 2:...Aufzeichnung eines abdominellen, fetalen EKG (Monica AN24 ${ }^{\mathrm{TM}}$ ) (S.18) Abbildung 3: EKG-Kurve, abgebildet durch die Software Monica DK (S.18)

Abbildung 4: Positionierung der Messelektroden im fetalen EKG (S.20) Abbildung 5: Das Monica AN24 ${ }^{\mathrm{TM}}$ Gerät (S.21)

Abbildung 6: (PRSA Signalverarbeitung) Überprüfung der Filterbedingung (S.24) Abbildung 7:... (PRSA Signalverarbeitung) Punkte innerhalb eines Tachogramms (S.24) Abbildung 8: .. Phasengleichrichtung der Tachogramme(A) u. Signalmittelung(B) (S.25) Abbildung 9: Berechnung der AAC (S.25) Abbildung 10: Box-Plots: STV in CTG und Monica (S.35) Abbildung 11: Box- Plots: AAC in CTG und Monica (S.36) Abbildung 12: $\quad$ Bland-Altman-Plot: Korrelation der STV im CTG: Oh und 24h (S.39) Abbildung 13: ........... Bland-Altman-Plot: Korrelation der STV im CTG Oh vs.48h (S.39) Abbildung 14: .......... Bland-Altman-Plot: Korrelation der STV im CTG Oh vs. 96h (S.40) Abbildung 15: ........... Bland-Altman-Plot: Korrelation der AAC im CTG: Oh vs.24h (S.41) Abbildung 16: ........... Bland-Altman-Plot: Korrelation der AAC im CTG: Oh vs.48h (S.41) Abbildung 17: ........... Bland-Altman-Plot: Korrelation der AAC im CTG: Oh vs.96h (S.42) Abbildung $18 \ldots . .$. Bland-Altman-Plot: Korrelation der STV im Monica: Oh und 24h (S.43) Abbildung 19: .... Bland-Altman-Plot: Korrelation der STV im Monica: Oh und 48h (S.43) Abbildung 20: .... Bland-Altman-Plot: Korrelation der STV im Monica: Oh und 96h (S.44) 
Abbildung 21: .....Bland-Altman-Plot:Korrelation der AAC im Monica: Oh und 24h (S.45)

Abbildung 22: ......Bland-Altman-Plot:Korrelation der AAC im Monica:0h und 48h (S.45)

Abbildung 23: .....Bland-Altman-Plot:Korrelation der AAC im Monica: Oh und 96h (S.46)

Abbildung 24: Streudiagramm: STV im Monica: Werte Oh und 96h (S.70)

Abbildung 25: Streudiagramm: STV und AAC im CTG zum Zeitpunkt Oh (S.71) 


\section{Tabellenverzeichnis}

Tabelle 1: Klinische Charakteristika der Studienteilnehmerinnen (S.27)

Tabelle 2: Indikation zur Betamethason-Gabe (S.28)

Tabelle 3: Differenz der Mittelwerte zwischen den Messzeitpunkten (S.30)

Tabelle 4: Ergebnisse der T-Tests für gepaarte Stichproben: STV im CTG (S.31)

Tabelle 5: Ergebnisse der T-Tests für gepaarte Stichproben: AAC im CTG (S.32)

Tabelle 6: Ergebnisse der T-Tests für gepaarte Stichproben: STV im Monica (S.33)

Tabelle 7: Ergebnisse der T-Tests für gepaarte Stichproben: AAC im Monica (S.34)

Tabelle 8: Übersicht der Korrelationskoeffizienten aller Parameter vor und nach Betamethasongabe (S.37)

Tabelle 9: Korrelation der Ausgangswerte zu den Werten weiterer Messzeitpunkte: STV im CTG (S.38)

Tabelle 10: Korrelation der Ausgangswerte zu den Werten weiterer Messzeitpunkte: AAC im CTG (S.40)

Tabelle 11: Korrelation der Ausgangswerte zu den Werten weiterer Messzeitpunkte: STV im fetalen EKG (S.42)

Tabelle 12: Korrelation der Ausgangswerte zu den Werten weiterer Messzeitpunkte: AAC im fetalen EKG (S.44)

Tabelle 13: Korrelation der STV und AAC im CTG (S.46)

Tabelle 14: Korrelation der STV und AAC im fetalen EKG (S.47)

Tabelle 15: Korrelation der STV in CTG und fetalem EKG (S.48)

Tabelle 16: Korrelation der AAC in CTG und fetalem EKG (S.49) 
Tabelle 17: Signalverlust zu den verschiedenen Messzeitpunkten (S.51)

Tabelle 18: Phasen der intrauterinen Lungenentwicklung (S.111) 


\section{$9 \quad$ Anhang}

\subsection{Studienlage zur iatrogenen Induktion der Lungenreifung mit Glucocorticoiden}

\subsubsection{Empfehlung der Leitlinien}

Die Leitlinien der deutschen Gesellschaft für Gynäkologie und Geburtshilfe, sowie die Leitlinien des Royal College of Obstetricians and Gynaecologists empfehlen im Jahr 2014 hinsichtlich der antenatalen Verabreichung von Glucocorticoiden zur Induktion der Lungenreife als Behandlungsregime, die Gabe von 12 $\mathrm{mg}$ Betamethason intramuskulär mit einer einmaligen Wiederholung nach 24 Stunden. Die britischen Leitlinien nennen alternativ die viermalige Gabe von 6mg Dexamethason intramuskulär im Abstand von 12 Stunden (vgl. AWMFLeitlinien der deutschen Gesellschaft für Gynäkologie und Geburtshilfe: Antenatale Kortikosteroide zur Lungenreifung, Stand Januar 2014, die Leitlinien befinden sich derzeit in Bearbeitung, vgl. Leitlinien des Royal College of Obstetricians and Gynaecologists: Antenatal Corticosteroids to Reduce Neonatal Morbidity and Mortality, Stand: Dezember 2014).

Sowohl deutsche als auch die britischen Leitlinien setzen sich bei der Empfehlung mit der Frage nach einer eventuellen Wiederholung der Gabe, sowie dem zu verwendenden Steroid auseinander. Beta- und Dexamethason stellen hier die beiden Hauptvertreter dar.

\subsubsection{Evidenz für Einzelgabe}

In einer Metaanalyse von zwölf randomisierten Studien konnte Crowley 1990 zeigen, dass durch die antenatale Verabreichung von Kortikosteroiden die neonatale Mortalität sowie das Auftreten von RDS und IVH beim Neugeborenen gesenkt werden konnten. Im Hinblick auf das Auftreten des RDS profitierten jene Kinder am stärksten, die im Intervall zwischen 24 Stunden und sieben Tagen nach erfolgter Glucocorticoidgabe geboren wurden. 
In der Cochrane- Analyse von 2006 konnten Roberts und Dalziel für die Inzidenz der cerebroventrikulären Hämorrhagien, des RDS sowie den Bedarf nach respiratorischer Unterstützung nach der Geburt in Form von mechanischer Ventilation oder CPAP-Beatmung(continuous positive airway pressure), ebenfalls eine mit der Kortikosteroidgabe assoziierte Reduktion nachweisen. Sie fanden ebenfalls eine Abnahme der neonatalen Mortalität. Für die Abnahme der kombinierten fetalen und neonatalen Mortalität konnte allerdings eine signifikante Reduktion nur bei Geburt innerhalb 24 Stunden nach Steroidapplikation nachgewiesen werden. In diesem Zusammenhang zeigte sich auch ein reduziertes Geburtsgewicht bei Kindern, die nach mehr als sieben Tagen nach Steroidgabe geboren wurden.

In der Analyse von Roberts und Dalziel zeigte sich darüber hinaus, dass mit der Gabe von Kortikosteroiden ein vermindertes Auftreten der NEC sowie systemischen Infektionen innerhalb von 48 Stunden postnatal assoziiert war.

Im Hinblick auf Langzeitbeobachtungen konnten im Kindesalter keine Unterschiede bezüglich der Parameter Mortalität und neurologische Entwicklungsverzögerung zwischen den Vergleichsgruppen festgestellt werden. Im Zusammenhang mit Kortikosteroidbehandlung traten tendenziell weniger häufig Fälle mit Cerebralparese (CP) auf.

Roberts und Dalziel konnten für das Outcome der Mutter zeigen, dass zwischen den Gruppen kein Unterschied in der mütterlichen Mortalität sowie dem Auftreten von Chorioamnionitis und Puerperalsepsis bestand. Die Outcomeparameter für das Neugeborene wurden auch für den Fall des Vorliegens von hypertensiven Schwangerschaftserkrankungen sowie vorzeitigen Blasensprung (premature rupture of membrane, PROM) untersucht. In Bezug auf das Vorliegen von hypertensiven Schwangerschaftserkrankungen zeigte sich, dass durch Steroidbehandlung eine signifikante Reduktion der neonatalen Mortalität sowie der der Inzidenz des RDS und cerebroventrikulären Hämorrhagien erzielt werden konnte. Beim Vorliegen von PROM zeigte sich für die unmittelbare Steroidgabe zum Zeitpunkt des PROM eine signifikante Reduktion der kombinierten fetalen und neonatalen Mortalität sowie der Inzidenz von cerebroventrikulären Hämorrhagien und NEC. Bei einem zeitlichen Abstand von mehr als 24 bzw. 48 Stunden 
zwischen PROM und Steroidgabe ließ sich dieser Effekt nicht mehr nachweisen. Für das RDS zeigte sich hingegen eine signifikante Reduktion durch Steroidgabe zum Zeitpunkt des PROM und bei Zurückliegen von mehr als 24 Stunden, nicht mehr jedoch ab einem Zeitintervall von mehr als 48 Stunden zwischen Steroidgabe und PROM (vgl. Roberts et al 2006).

\subsubsection{Vergleich von Betamethason und Dexamethason}

Strukturell unterscheiden sich die beiden Präparate durch eine Methylgruppe in Position 16 an Ring D. Beide sind plazentagängig, wirken schwach immunsuppressiv und haben nur geringe mineralocorticoide Restwirkung. Im fetalen Blut werden ca. 30\% der mütterlichen Wirkspiegel erreicht. Um eine therapeutische Wirkung zu erzielen, reichen verglichen mit Hydrocortison niedrigere Dosierungen aus.

Betamethason wird meist als Suspension aus Betamethason-Azetat und Betamethason-Natrium-Phosphat, einem löslichen Ester der schnell absorbiert wird, verabreicht. Dadurch weisen die Präparate eine unterschiedliche Pharmakokinetik auf: Betamethason hat verglichen mit Dexamethason eine längere Plasmahalbwertszeit und unterliegt geringeren Schwankungen in der Serumkonzentration (vgl. Bonanno 2009).

Bezüglich ihrer Wirkung auf Mutter und Kind werden stets mehrere Aspekte betrachtet. Dies sind zum einen die Auswirkungen auf den Fetus und die Parameter die zu seiner Überwachung in utero verwendet werden. Zum anderen das postnatale Outcome des Kindes. Hierbei betrachtet man verschiedene Outcomeparameter unmittelbar nach der Geburt bis hin zur Langzeitbeobachtung nach mehreren Jahren. Darüber hinaus werden geburtshilfliche sowie internistische Konditionen der Mutter, die vor einer eventuellen Kortikosteroidgabe berücksichtigt werden müssen, untersucht sowie die Auswirkung der Steroidgabe auf den mütterlichen Organismus.

\subsubsection{Auswirkung der antenatalen Steroidgabe auf den Feten}

Es existieren verschiedene Parameter, um das Wohlbefinden des Kindes in utero einzuschätzen. Diese Parameter werden durch Kortikosteroidgabe auf 
verschiedene Art und Weise beeinflusst. Es ist wichtig, umfassende Erkenntnisse über diese Auswirkungen zu erlangen, da die Beurteilung der Parameter in der weiteren geburtshilflichen Entscheidungsfindung, insbesondere über eine eventuell vorzeitige Entbindung, eine wichtige Rolle spielen.

Neben der Beurteilung des biophysikalischen Profils des Feten mittels Sonografie und der der Flussprofile in verschiedenen Gefäßen mittels Dopplersonografie, ist es möglich, die fetale Herzfrequenz mittels Kardiotokographie, sowie fetaler Elektrokardiografie zu analysieren. Die Auswirkungen der Betamethasongabe auf die fetale Herzfrequenzvariabilität werden im Abschnitt 4.2.1. „Einfluss der antenatalen Kortikosteroidgabe zur Lungenreifeinduktion auf die fetale Herzfrequenzvariabilität" diskutiert.

Der Einfluss der Kortikosteroidgabe auf das biophysikalische Profil und vaskuläre Flussprofile wurde vielfach untersucht.

Mulder und Derks berichteten neben der veränderten Herzfrequenzvariabilität über eine reversible Abnahme der Atem-und Körperbewegung in Folge von Kortikosteroidgabe. Besonders ausgeprägt war der Effekt nach Betamethason (vgl. Mulder et al 1997).

Rotmensch et al stellten sowohl für mehrere Parameter der fetalen Herzfrequenz als auch für die Anzahl der Gesamtbewegungen des Kindes, der Anzahl fetaler Atmungsepisoden, sowie Glieder- und Rumpfbewegungen eine reversible Abnahme 48 Stunden nach Kortikosteroidapplikation fest. Auch hier war nach Betamethason- für die Anzahl der Gesamtbewegungen eine stärkere Abnahme als nach Dexamethasongabe zu beobachten.

Für Pulsatilitäts-Indices der Umbilikal-, sowie cerebraler Arterien, fanden sich keine Veränderung (vgl. Rotmensch et al 1999a und 1999b). Kelly et al beschrieben ebenfalls Auswirkungen der Betamethasongabe auf das biophysikalische Profil und kamen zu dem Schluss, das durch Glucocorticoide das biophysikalische Profil zwar beeinflusst wird, dahinter jedoch keine relevante Verschlechterung der fetalen Situation in utero steht (vgl. Kelly et al 2000). Cohlen et al fanden in Doppleruntersuchungen nach Betamethasongabe ebenfalls keinerlei Hinweise auf Veränderungen in Flussprofilen verschiedener untersuchter 
fetaler Gefäße unter Betamethasoneinfluss. Sie nehmen daher an, dass beobachtete Veränderungen unter Kortikoideinfluss demnach nicht auf eine durch die Glucocorticoidgabe induzierte fetale Hypoxie zurückzuführen ist (vgl. Cohlen et al 1996).

\subsubsection{Auswirkung der antenatalen Steroidgabe auf das Neugeborene}

Baud et al untersuchten den Einfluss der Kortikosteroide auf die Inzidenz der cystischen, periventrikulären Leukomalazie (CPVL), eine der Hauptursachen für Cerebralparese im Kindesalter. Sie fanden unter Betamethasongabe eine niedrigere Rate an CPVL als bei Gabe von Dexamethason oder bei keinerlei Steroidexposition (vgl. Baud et al 1999).

Roberts und Dalziel fanden bezüglich des Einfluss auf die Inzidenz der cerebroventrikulären Hämorrhagie keinen Unterschied zwischen den Präparaten. Allerdings stellten sie für das Auftreten des RDS eine stärkere Reduktion durch $\mathrm{Be}$ tamethason, als durch Dexamethason fest. Dies könnte allerdings durch eine erhöhte Hintergrundprävalenz des RDS in den Gruppen, die Dexamethason erhielten, verursacht sein. In über 14 untersuchten Studien mit 2563 Kindern lag das Relative Risiko (RR) für das RDS unter Betamethasoneinfluss bei 0,56 $(95 \% \mathrm{Cl}=[0.48-0.65])$. In über sechs Studien mit 1457 Kindern lag das Ergebnis

für Dexamethason hingegen bei einem Relativen Risiko von 0.80 $(95 \% \mathrm{Cl}=[0.68-0.93])$ (vgl. Roberts et al 2006).

Lee et al untersuchten die Auswirkungen der Steroidgabe bei Kindern mit verylow-birth-weight (VLBW). Für das Auftreten der periventrikulären Leukomalazie zeigte sich im Vergleich der beiden Präparate kein unterschiedlich hohes Risiko. Die neonatale Mortalität reduzierte sich unter der Gabe von Betamethason signifikant. Sie war im Vergleich mit der Gabe von Dexamethason mit einer stärkeren Reduktion der neonatalen Mortalität assoziiert (vgl. Lee et al 2006).

Eine weitere Untersuchung an Kindern mit VLBW konnte signifikant niedrigere Raten an RDS und bronchopulmonaler Dysplasie (BPD) unter Betamethasongabe, verglichen mit Dexamethasongabe nachweisen: die Rate an RDS in der Betamethasongruppe betrug $62,9 \%$, in der Dexamethasongruppe $81.8 \%$ $(p<0.001)$. Die Rate an BPD in der Betamethasongruppe lag bei $43 \%$, vergli- 
chen mit der Dexamethasongruppe, in der eine Rate an $54.7 \%(p<0.03)$ zu finden war (vgl. Feldman et al 2007).

In der sog. Betacode-Trial, der einzigen kontrolliert, randomisierten Studie, die die beiden Kortikosteroide miteinander vergleicht, konnte kein signifikanter Unterschied zwischen den Präparate in Bezug auf das Auftreten von RDS, NEC, neonatal Mortalität, sowie neonataler Sepsis festgestellt werden. Die Rate an IVH konnte jedoch durch Dexamethasongabe effektiver gesenkt werden als durch Betamethasongabe. Mit einer Häufigkeit von 5.7\% innerhalb der Dexamethasongruppe lag, verglichen mit $17 \%$ in der Betamethasongruppe, das Relative Risiko bei $2.97(95 \% \mathrm{Cl}=[1.22-7.24]$, p<0.02) (vgl. Elimian et al 2007).

Brownfoot et al bekräftigten 2008 in einem Cochrane Review dieses Ergebnis. In vier beobachteten Studien mit insgesamt 549 Kindern zeigte sich eine stärkere Senkung der Inzidenz der IVH durch Dexamethasongabe als durch Betamethasongabe. Es konnten ebenfalls keine statistisch signifikanten Unterschiede für das Auftreten des RDS, BPD, NEC und dem mittleren Geburtsgewicht gefunden werden (vgl. Brownfoot et al 2008).

In der Empfehlung von Jobe und Soll 2004 wird Betamethason, insbesondere unter dem Aspekt der neonatalen Sterblichkeit, zur Verwendung bei Frauen mit drohender Frühgeburt empfohlen. Die deutschen Leitlinien empfehlen im Jahr 2014 ebenfalls die Gabe von Betamethason. Einige Autoren merken jedoch an, dass sich aus der momentanen Datenlage nicht genug Anhalt ergibt, um ein Präparat klar gegenüber dem anderen zu empfehlen (vgl. Bonanno 2009).

\subsubsection{Auswirkung der antenatalen Steroidgabe auf die Langzeitentwicklung des Kindes}

Smolders-de Haas et al untersuchten 1990 Kinder im Alter von zehn bis zwölf Jahren in Gruppen, deren Mütter entweder einmalig Betamethason oder Placebo in der Schwangerschaft erhalten hatten. Fremdanamnestisch fanden sich in der Gruppe der Steroidtherapie mehr Hospitalisationen der Kinder in den ersten Lebensjahren auf Grund von Infektionskrankheiten (vgl. Smolders-de Haas 1990). 
Dessens et al untersuchten Kinder von Müttern nach Einmalgabe von Betamethason zur RDS-Prävention nach 20 Jahren im Hinblick auf Wachstum, kognitive Funktion, Geschlechtsentwicklung, psychosexuelle Entwicklung, Bildungsstandard, sozioökonomischen Status und weitere Punkte. Es zeigten sich keine nachteiligen Effekte durch die Betamethasongabe nach 20 Jahren (vgl. Dessens et al 2000).

Haynes et al konnten im Tierexperiment schädliche Auswirkungen von Dexamethason auf bestimmte Zellpopulationen in Striatum sowie Hippocampus nachweisen. Diese Schäden könnten bei sog. „mood disorders“, bei denen erhöhte Cortisol-Serumkonzentrationen beobachtet werden, zu psychotischen oder depressiven Symptomen dieser Erkrankungen beitragen (vgl. Haynes et al 2001).

Roberts und Dalziel fanden keinerlei Hinweise auf Beeinträchtigungen durch pränatale Glucocorticoidgabe in der Kindheit im Hinblick auf Mortalität sowie neurokognitive Entwicklung (vgl. Roberts et al 2006).

Lee et al verglichen die neurologische Entwicklung von Kindern mit VLBW im Alter von 18-22 Monaten nach dem ihre Mütter entweder Betamethason, Dexamethason oder keinerlei Kortikosteroid während der Schwangerschaft erhalten hatten. Wie auch von Wapner 2007 (vgl. Kapitel 9.1.8. „Wiederholte Glucocorticoidgabe") wurde dazu der Mentale und Psychomotorische Entwicklungsindex der "Bayley Scales of Infant Development" verwendet. Außerdem wurden die Kinder auf das Vorliegen einer Cerebralparese (CP), Blindheit oder einer Beeinträchtigung der Hörfähigkeit untersucht. Lag mehr als einer der genannten Punkte bei den Kindern vor, so wurde dies als Beeinträchtigung der neurologischen Entwicklung bewertet. Im Hinblick auf die Indices zählten hier jeweils weniger als 70 erreichte Punkte. In der Untersuchung der insgesamt 1124 Kinder, die in die Studie eingeschlossen wurden, zeigte sich, dass die Gabe von Betamethason im Vergleich zu keinerlei Steroidgabe während der Schwangerschaft, mit einem reduzierten Risiko für eine Beeinträchtigung in der neurologischen Entwicklung assoziiert war. Im Vergleich mit Dexamethasongabe zeigte sich auch ein geringeres Risiko für Hörbeeinträchtigung bei den Kindern. Dexamethason war im Vergleich mit Betamethason außerdem mit einem 
höheren Risiko assoziiert, im psychomotorischen Entwicklungsindex weniger als 70 Punkte zu erzielen.

Spinillo et al 2004, Crowther et al 2007 sowie Wapner et al 2007 untersuchten die Langzeitauswirkungen der Kortikosteroidgabe vor allem hinsichtlich des Vergleiches mit Regimen, die wiederholte Steroidgaben verwendeten. Die Ergebnisse dieser Studien werden daher im Kapitel 9.1.8. „Wiederholte Glucocorticoidgabe: Evidenz und Auswirkungen" besprochen.

\subsubsection{Kortikosteroideinfluss auf den mütterlichen Organismus}

Roberts und Dalziel konnten, wie bereits beschrieben, auch bei Vorliegen hypertensiver Schwangerschaftserkrankungen oder PROM zeigen, dass die Gabe von Kortikosteroiden die neonatale Mortalität, die Inzidenz des RDS und cerebroventrikulärer Hämorrhagien sowie verschiedene weitere kindliche Morbiditäten günstig beeinflussen (vgl. Roberts et al 2006). Allerdings hat die Kortikosteroidgabe auch Auswirkungen auf den mütterlichen Organismus selbst. Roberts und Dalziel fanden unter Dexamethasongabe eine erhöhte Anzahl an Fällen mit Puerperalsepsis (vgl. Roberts et al 2006).

Auswirkungen auf die Inzidenz des Gestationsdiabetes bei Frauen mit drohender Frühgeburt wurden u.a. 1997 von Fisher et al untersucht. In Kombination mit der Gabe von Betamimetika konnte, im Vergleich mit der Kontrollgruppe, eine signifikant höhere Inzidenz des Gestationsdiabetes festgestellt werden. Die Gabe von Kortikosteroiden alleine führte lediglich zu einem Trend in Richtung einer erhöhten Inzidenz, war jedoch nicht signifikant (vgl. Fisher et al 1997).

Rotmensch et al wiesen für Frauen, die mehr als drei Gaben Betamethason erhalten hatten eine Assoziation dieser wiederholten Gaben mit einem erhöhten Risiko für insbesondere bakterielle Infektionen nach ( $\mathrm{vgl}$. Rotmensch et al 1999c).

\subsubsection{Wiederholte Glucocorticoidgabe: Evidenz und Auswirkungen}

Die Frage wie lange der Effekt der Glucocorticoidgabe und damit der Nutzen für das Neugeborene anhalten, wird kontrovers diskutiert. 
Einerseits gibt es Anhalt dafür, dass bei einem zeitlichen Abstand der Glucocorticoid Gabe von mehr als sieben Tagen zum Zeitpunkt der Geburt, durch die zurückliegende Gabe kein Vorteil mehr für das Neugeborene in Bezug auf fetale und neonatale Mortalität, das Auftreten von RDS und IVH mehr festgestellt werden kann (vgl. Roberts et al 2006). Andere Studienergebnisse geben jedoch Hinweis darauf, dass besonders in Abwägung mit eventuellen schädlichen $\mathrm{Ne}-$ benwirkungen wiederholter Gaben, auch über die Grenze von sieben Tagen hinaus bereits durchaus ausreichende Effekte erzielt wurden und eine erneute Gabe nicht zwingend erforderlich ist (vgl. Peaceman et al 2005).

Aus den unterschiedlichen Studiendaten ergeben sich daher teilweise Vorgehensweisen wie die erneute, sog. "rescue“- Gabe in den USA (vgl. ACOG Committee Opinion Februar 2011). Sowohl in Deutschland als auch in den USA wird jedoch eine definitive, wiederholte Gabe zu einem bestimmten Zeitpunkt von den Leitlinien derzeit nicht empfohlen (vgl. ACOG Committee Opinion Februar 2011, vgl. AWMF-Leitlinien der deutschen Gesellschaft für Gynäkologie und Geburtshilfe: Antenatale Kortikosteroide zur Lungenreifung, Stand Januar 2014).

Das 2007 veröffentlichte Cochrane Review zur Frage nach wiederholten Gaben kommt zu dem Ergebnis, dass durch wöchentliche Gaben weniger häufig schwere neonatale Lungenerkrankungen, insbesondere das RDS, auftreten. Allerdings wirken sich die wiederholten Gaben auch hinsichtlich einer Reduktion des Geburtsgewichtes (Z-scores) bzw. einer steigenden Inzidenz von SGANeugeborenen aus. Kein Unterschied ließ sich hinsichtlich Mortalität, Inzidenz der PVH, PVL sowie mütterliche Infektionen finden (vgl. Crowther et al 2007a).

In insgesamt 4 Studien mit 1523 Teilnehmerinnen zeigte sich ein signifikant erhöhtes Risiko für die Inzidenz einer Sectio caesare nach wiederholter Kortikosteroidgabe (vgl. Crowther et al 2007a).

Crowther et al betonten die Bedeutung von noch unzureichenden Langzeitdaten, die Auskunft über die körperliche und kognitive Entwicklung der Kinder durch die pränatale Kortikosteroidapplikation geben können. 
Existierende Langzeitbeobachtungen von Kindern, deren Mütter wiederholte Kortikosteroidgaben in der Schwangerschaft erhalten haben, beschäftigen sich vorrangig mit der Frage nach dem Einfluss der wiederholten Gaben auf Körpergröße, Gewicht, Kopfumfang, respiratorische Morbidität sowie dem neurologischen Status und kognitiven Beeinträchtigungen im Langzeitverlauf. Der neurologische Status sowie die kognitive Entwicklung werden anhand sensorische Defizite wie Blindheit oder Taubheit, der Inzidenz der Cerebralparese (CP), sowie Anhand der psychomotorischen und mentalen Entwicklung validiert.

Wapner kam 2007 zu dem Ergebnis, dass nach zwei Jahren keine Unterschiede in der Entwicklung von Gewicht, Größe und Kopfumfang sowie der neurokognitiven Entwicklung besteht. Letztere wurde anhand des erzielten Scores im Psychomotorischen und Mentalen Entwicklungs Index der „Bayley Scales of Infant Development" evaluiert.

Die Anzahl der Fälle mit Cerebralparese lag in der Gruppe der wiederholten Gaben allerdings, wenn auch nicht signifikant, höher als in der Gruppe der Einzelgaben (vgl. Wapner et al 2007).

Die ACTORDS Study Group (australian collaborative trial of repeat doses of steroids) um Crowther et al fand 2007 in einer Nachuntersuchung bei Kindern im Alter von zwei Jahren keinen Unterschied zur Vergleichsgruppe bezogen auf die Körpergröße und respiratorische Morbidität. Allerdings gab es Hinweise auf eine verminderte Aufmerksamkeit der Kinder nach wiederholter Steroidgabe (vgl. Crowther et al 2007b).

French et al beschrieben 2003 in einer nicht-randomisierten Studie, in der Kinder im Alter von drei und sechs Jahren nachuntersucht wurden, ebenfalls Auswirkungen wiederholter Gaben auf das Verhalten. Es zeigte sich eine vermehrte Ablenkbarkeit der Kinder und vermehrt hyperkinetische und aggressive Verhaltensmuster (vgl. French et al 2004).

Wichtige Hinweise auf eventuelle Auswirkungen wiederholter Kortikosteroidapplikation liefern darüber hinaus Tierstudien.

Ikegami und Jobe zeigten, dass die wöchentliche Gabe von Betamethason das Geburtsgewicht von Schafen verringerte und Einfluss auf endokrinologische 
Parameter wie veränderte Blutkonzentrationen von Epinephrin und thyreoidalen Hormonen hat (vgl. Ikegami et al 1997).

Der Einfluss der Steroidgabe auf die Regulation der Hypothalamus-HypophyseNebennierenrinden-Achse nach der Geburt, wurde sowohl von Sloboda et al an Schafen bis zu sechs bzw. zwölf Monate postpartal nachgewiesen (vgl. Sloboda et al 2002), als auch von Banks et al am Menschen untersucht. Hier zeigte sich ein im Vergleich erniedrigter Kortisonspiegel zwei Stunden nach der Geburt im Vergleich von einmaliger und mehrmaliger Kortikosteroidgabe, was als Hinweis auf eine prolongierte Nebennierenrinden-Suppression nach mehrmaliger Gabe interpretiert werden könnte (vgl. Banks et al 1999).

Die wiederholte Applikation von Betamethason wirkt sich auch verzögernd auf die Reifung der Retina bei Schafen aus, wie Quinlivan et al zeigen konnten (vgl. Quinlivan et al 2000).

Huang et al konnten wiesen nach, dass sowohl die einfache als auch die wiederholte Gabe von Glucocorticoiden einen Einfluss auf das Wachstum des fetalen Gehirns bei Schafen besitzt (vgl. Huang et al 1999).

Mehrere Studien beschäftigten sich mit der Auswirkung einer wiederholten, präpartalen Applikation von Betamethason auf die Myelinisierung von Strukturen des zentralen Nervensystems. Dunlop et al konnten zeigen, dass dadurch die Myelinisierung der Axone des N.opticus bei Schafen verzögert wird (vgl. Dunlop et al 1997). Huang et al beschrieben den gleichen Effekt bezogen auf das Corpus Callosum, welches in wichtigen kognitiven Prozessen eine Rolle spielt (vgl. Huang et al 2001a).

Der Einfluss der Kortikosteroide auf die Struktur der Blut-Hirn-Schranke (BHS) ist ebenfalls von zentraler Bedeutung. Die BHS setzt sich aus dem Endothel cerebraler Gefäße, Astrozyten und den zwischen diesen ausgebildeten TightJunctions zusammen. Es wird vermutet, dass der Einfluss von Kortikosteroiden auf das Auftreten der IVH im Zusammenhang mit induzierten Reifungsprozessen der Bluthirnschranke steht. Der genaue protektive Mechanismus durch Kortikosteroide ist allerdings unklar (vgl. Quinlivan et al 2002). Sie konnten darüber hinaus nachweisen, dass Betamethason nach wiederholter Gabe die Konzent- 
ration des GFAP (glial fibrillary acidic protein) verringert, ein Protein das in Astrozyten vorkommt und am Aufbau der subendothelialen Komponente der BHS beteiligt ist (vgl. Quinlivan et al 2002). Huang et al beschrieben eine verzögerte Ausbildung astrozytärer Tight-Junctions bei fetalen Schafen nach wiederholter Gabe von Betamethason (vgl. Huang et al 2001).

\subsection{Die fetale Lunge}

\subsubsection{Entwicklung der fetalen Lunge}

Die Entwicklung der fetalen Lunge gliedert sich in vier intrauterine Entwicklungsabschnitte und setzt sich postnatal in der alveolären Phase fort. In den ersten beiden bilden sich die luftleitenden, in den letzten beiden die respiratorischen Anteile des pulmonalen Systems, sowie die Blut-Gas-Schranke aus, die das Neugeborene zum Gasaustausch in den Alveolen befähigt.

Tabelle 18 gibt einen Überblick über die zeitliche Einordnung der Abschnitte in die Schwangerschaftsverlauf, sowie die wesentlichen Entwicklungsvorgänge zu den entsprechenden Zeitpunkten.

Die Lunge eines Neugeborenen hat etwa 150 Millionen Alveolen. Innerhalb der ersten zwei Lebensjahre verdoppelt sich diese Anzahl (vgl. Bonanno 2008). Zusammen mit den innen vorgeschalteten Ductus alveolares und Bronchioli respiratorii, sowie den Bronchioli terminales, bilden sie die kleinste morphologische Einheit des Gasaustausches: den Azinus. Der Azinus entspricht demnach der Verzweigungseinheit des Bronchialsystems ab dem Bronchius terminalis.

Die maximale Reifung der sog. Sakkuli terminales, die jedem Ductus alveolaris nachgeschaltet sind, zu den Alveolen, erfolgt erst postnatal. Sie entsprechen also der intrauterinen Vorstufe der Alveolen.

Der Gasaustausch in den Alveolen erfolgt über die Blut-Gas-Schranke. Diese setzt sich in ihrer reifen Form aus drei Strukturen zusammen: Dem Kapillarendothel, dem Typ I Pneumozyten mit umgebendem Zytoplasmasaum und dem Interstitium. Die Größe der Gasaustauschfläche bei der Geburt spielt eine 
wichtige Rolle, insbesondere für die Überlebensfähigkeit von sehr unreifen Frühgeborenen.

Die Blut-Gas-Schranke ist fokal bereits ab der 20.SSW in solchen Bereichen der Lunge nachweisbar, in dem Kapillarendothel mit respiratorischem Epithel in enge Verbindung kommt. Die wöchentliche Entwicklung ihrer Gesamtfläche zeigt ab diesem Zeitpunkt einen exponentiellen Verlauf. Ein effektiver Gasaustausch findet jedoch wahrscheinlich erst ab der sakkulären Phase statt. Hier besteht jedoch eine interindividuelle Variabilität, so dass in seltenen Fällen auch bereits bei Geburt vor der 24. SSW ein ausreichender, pulmonaler Gaswechsel durch supportive Maßnahmen erzielt werden kann.

Histologisch lassen sich sechs Stadien der Lungenreife, die mit der Azinusentwicklung eng korrelieren, definieren.

Hierbei sind der Gehalt des kubischen Epithels postbronchiolär, die Breite der intersakkulären Septen, die Beurteilung der Anzahl der in innen verlaufenden Kapillaren sowie ihrer Beziehung zum respiratorischen Epithel mit seinen spezialisierten Zellen entscheidend.

Eine histologisch unreife Lunge bedeutet morphologische und somit funktionelle Defizite für das Frühgeborene. Beispielsweise bewirkt die Involution der Bindegewebssepten in der sakkulären Phase auch die Vergrößerung potenzieller Atemräume, die postnatal zur Verfügung stehen, auch wenn die Lunge bereits zum Gasaustausch befähigt sein kann.

Die zelluläre Differenzierung des Epithels beginnt im Hinblick auf die Ausbildung neuroendokrinen Zellen, Flimmerepithel, Becher- und Clarazellen bereits ab der mittleren Embryonalperiode.

Die Differenzierung der Pneumozyten Typ I und II, welche am Aufbau der Alveolaroberfläche entscheidend beteiligt sind, erfolgt später, wobei sich die Pneumozyten Typ I vorwiegend in der sakkulären Phase entwickeln.

Die Pneumozyten Typ II sind für die Synthese des sog. Surfactant verantwortlich. Sie erfahren bereits ab der 20./21. SSW eine intensive Differenzierung. Die Surfactant- Synthese erfolgt im endoplasmatischen Retikulum und unterliegt hormonellen Regulationsmechanismen. Er wird intrazellulär in sog. Lamellar- 
körperchen gespeichert, diese Speicherorganellen sind das strukturelle Korrelat der Surfactantsynthese und charakteristisches, morphologische Merkmal der Pneumozyten Typ II. Die Lamellarkörperchen lassen sich mit fortschreitender Differenzierung der Pneumozyten Typ II, ab der 20./21.SSW regelmäßig nachweisen. Frühe Vorstufen können jedoch bereits ab der embryonalen Phase beobachtet werden. Durch Fusion der Lamellarkörperchen mit der Zellmembran wird das Surfactant ins Lumen der Alveole freigesetzt. Alveolarmakrophagen, die für die alveoläre Clearance verantwortlich sind, räumen auch überschüssiges Surfactantmaterial ab. Da sie teilweise in das Interstitium rezirkulieren, erfolgt hierüber ein endogener „Turnover“ des Surfactants (vgl. Vogel 2004).

\begin{tabular}{|c|c|c|}
\hline Embryonale Phase & $\begin{array}{l}\text { Ende der } 3 . \\
\text { Woche p.c.- } \\
\text { 10.SsW }\end{array}$ & $\begin{array}{l}\text { „Primäraufbau der Lunge“ Bildung der La- } \\
\text { ryngotrachealrinne, die sich zur Trachea } \\
\text { entwickelt; Entwicklung der Lungenknospen } \\
\text { aus denen die Segmentbronchien entstehen; } \\
\text { Pulmonalarterien-Entwicklung, diese folgt der } \\
\text { Entstehung des Bronchialsystems. }\end{array}$ \\
\hline $\begin{array}{l}\text { Pseudoglanduläre } \\
\text { Phase } \\
\text { „Histologisch Ähnlich- } \\
\text { keit mit Drüsengewe- } \\
\text { be“ }\end{array}$ & 10.-17.SSW & $\begin{array}{l}\text { Verzweigung des Bronchialsystems mit } \\
\text { Entwicklung präazinärer Bronchialanteile mit } \\
\text { folgender Vaskularisierung; der hier erreichte } \\
\text { Verzweigungsgrad des Bronchialsystems } \\
\text { entspricht dem bei der Geburt. }\end{array}$ \\
\hline Kanalikuläre Phase & 18.-24.SSW & $\begin{array}{l}\text { Formung der Azinuseinheit: Entstehung } \\
\text { der terminalen und respiratorischen Bronchi- } \\
\text { oli mit Ductus alveolares und terminalen } \\
\text { Sakkuli, den späteren Alveolen. Vaskulari- } \\
\text { sierung erfolgt bis einschließlich den respira- } \\
\text { torischen Bronchiolii. Schwerpunkt der Diffe- } \\
\text { renzierung der Pneumozyten Typ II ab der } \\
\text { 21. SSW, ab diesem Zeitpunkt sind Lamel- } \\
\text { larkörperchen regelmäßig nachweisbar }\end{array}$ \\
\hline Sakkuläre Phase & 24.SSW bis & Formierung der Blut-Gas-Schranke u. Bil- \\
\hline
\end{tabular}




\begin{tabular}{|l|l|l|}
\hline 2.LJ postnatal & $\begin{array}{l}\text { dung der Typ I Pneumozyten: Bindege- } \\
\text { webssepten zwischen den Sakkuli sowie das } \\
\text { Sakkulusepithel werden dünner, Kontakt zwi- } \\
\text { schen respiratorischen Strukturen und Kapil- } \\
\\
\end{array} \quad \begin{array}{l}\text { laren vergrößert sich, insbesondere zwischen } \\
\text { Kapillaren und dem Epithel der Sakkuli ter- } \\
\text { minales. }\end{array}$ \\
\hline
\end{tabular}

Tabelle 18: Phasen der intrauterinen, fetalen Lungenentwicklung. P.c.=post conceptionem, SSW=Schwangerschaftswoche, LJ=Lebensjahr.

\subsubsection{Physiologische Lungenreifung und Bedeutung der Glucocorticoide}

Die Reifung der fetalen Lunge bis zur Fähigkeit zum suffizienten postnatalen Gasaustausch beinhaltet sowohl strukturelle Veränderungen der Blut-GasSchranke, als auch die ausreichende Bildung von Surfactant. Diese Prozesse werden intrauterin vor allem durch endogene Glucocorticoide stimuliert.

Ein Mangel an Surfactant bei der Geburt in einer noch unreifen Lunge führt zum klinischen Bild des RDS (Respiratory distress syndrome). Dieser pathophysiologische Zusammenhang wurde 1959 von Avery und Mead zum ersten Mal beschrieben (vgl. Avery 1959).

Das Krankheitsbild des RDS ist die Hauptursache früher, neonataler Mortalität (vgl. Roberts et al 2006). Die respiratorische Insuffizienz nach der Geburt führt unbehandelt durch progressive Hypoxie und schließlich respiratorisches Versagen zum Tode des Neugeborenen (vgl. Sweet et al 2007).

Pulmonales Surfactant setzt sich aus Surfactantproteinen, sowie Phospholipiden zusammen. Durch die spezifischen Eigenschaften dieser Komponenten bewirkt es die Herabsetzung der Oberflächenspannung in den Alveolen auch unter sich ändernden Lungenvolumina während des Atmungsvorgangs. Es wirkt somit antiatelektatisch (vgl. Stevens 2004, S.18).

Für diese stabilisierende Funktion sind hauptsächlich die Surfactantproteine B und $C$ sowie DP-Phosphatidylcholin, das den größten Anteil an den Phospholipiden ausmacht, verantwortlich (vgl. Stevens 2004 S.11). Die Surfactantprotei- 
ne $A$ und $D$, die zu den Collectinen gehören, haben hingegen vor allem Bedeutung in der Infektabwehr (vgl. Stevens 2004 S.18, vgl. Bolt et al 2001).

Die Konzentration freier Glucocorticoide, die Menge ihrer Rezeptoren im pulmonalen Gewebe, sowie weitere Faktoren, welche die Bioverfügbarkeit der Glucocorticoide beeinflussen, steigen erst im Verlauf der Schwangerschaft an (vgl. Ballard et al 1995). Daher besteht insbesondere bei zu früh geborenen Kindern ein Defizit in der Glucocorticoidversorgung (vgl. Bolt 2001, S.84), was zu einer funktionell unreifen Lunge bei der Geburt führt.

Die Kenntnis dieses Zusammenhangs war Grundlage des heutigen etablierten Vorgehens, die Lungenreife bei drohender Frühgeburt durch iatrogene Gabe von Glucocorticoiden zu induzieren.

Nachdem Liggins 1969 im Tierexperiment zeigen konnte, dass die Verabreichung von Dexamethason einen Einfluss auf die Surfactantbildung bei Lämmern hat und zusammen mit Howie 1972 die erste Studie am Menschen zur Prävention des RDS mit Betamethason durchführte, wurde die Bedeutung der iatrogenen Glucocorticoidgabe auf die fetale Entwicklung, insbesondere im Hinblick auf die Lungenreifung, vielfach untersucht (vgl. Liggins 1969, vgl. Liggins et al 1972).

Crowley konnte 1990 in der ersten wegweisenden Metaanalyse zeigen, dass die antenatale Gabe von Glucocorticoiden effektiv in der Prävention des RDS und der Senkung der neonatalen Mortalität ist (vgl. Crowley 1990).

Im Jahr 2006 bestätigten Roberts und Dalziel in einem Cochrane Review über 21 Studien diese Ergebnisse. Die neonatale Mortalität wurde unter Glucocorticoidgabe, gegenüber Placebogabe oder keinerlei Behandlung, signifikant reduziert und die Inzidenz es RDS gesenkt. Das Ausmaß an neonataler, respiratorischer Unterstützung in Form von assistierter Beatmung, der Surfactantbedarf und die Dauer der Sauerstoffgabe waren signifikant verringert (vgl. Roberts et al 2006).

Neben der positiven Auswirkungen der Glucocorticoidgabe auf das respiratorische System, konnten sowohl Crowley et al als auch Roberts et al zeigen, dass sich das Risiko intraventrikulärer Hämorrhagien, durch vasokonstriktive Einflüs- 
se der Glucocorticoide, verringert. Roberts und Dalziel fanden darüber hinaus eine Abnahme der Inzidenz der nekrotisierenden Enterokolitis (vgl. Crowley et al 1990, vgl. Roberts et al 2006). 


\section{Danksagung}

Allen voran möchte ich meiner Betreuerin Frau Dr. med. Silvia Lobmaier danken, die mir die praktische Durchführung dieser Studie anvertraute. Für ihr Fachwissen, ihre konstruktive Kritik, weiterführenden Ideen und ansteckende Motivation über die gesamte Zeit.

Herzlichen Dank an Herrn Prof. Dr. med. K.-Th.-M. Schneider, für die Überlassung dieses interessanten und patientennahen Themas.

Die erfolgreiche Durchführung der Studie war nur möglich durch alle Ärzte und Hebammen der Frauenklinik des Klinikum rechts der Isar. Ihnen allen gilt mein ganz besonderer Dank.

Vielmals möchte ich auch Herrn Dipl.-Stat. Bernhard Haller für seine geduldige und kompetente Beratung in statistischen Fragen danken.

Von Herzen möchte ich meinen Freunden und meinem Bruder für die Anteilnahme und Unterstützung an meiner Arbeit danken. Insbesondere jedoch der Apothekerin Elisabeth Delitsch, deren Freundschaft in jeder Hinsicht Inspiration ist.

Der größte Dank von allen gilt meinen Eltern. Ihr habt mich zu einem neugierigen, selbstbewussten Menschen erzogen, das Medizinstudium ermöglicht und mir stets mit Liebe, Weisheit und Geduld zur Seite gestanden. Danke. 


\section{Erklärung}

Hiermit erkläre ich, dass ich die vorliegende Doktorarbeit selbständig angefertigt habe. Es wurden nur die in der Arbeit ausdrücklich benannten Quellen und Hilfsmittel benutzt. Wörtlich oder sinngemäß übernommenes Gedankengut habe ich als solches kenntlich gemacht.

Köln, den 14.12.2014

Ort, Datum

Unterschrift 